\title{
Photoreactions and Structural Changes of Anabaena Sensory Rhodopsin
}

\section{Akira Kawanabe and Hideki Kandori *}

Department of Frontier Materials, Nagoya Institute of Technology, Showa-ku, Nagoya, 466-8555, Japan; E-Mail: kawanabe.akira@nitech.ac.jp (A.K.)

* Author to whom correspondence should be addressed; E-Mail: kandori@ntiech.ac.jp; Tel.: +8-052-735-5207; Fax: +8-052-735-5207.

Received: 18 September 2009; in revised form: 14 October 2009 / Accepted: 23 October 2009 / Published: 3 December 2009

\begin{abstract}
Anabaena sensory rhodopsin (ASR) is an archaeal-type rhodopsin found in eubacteria. The gene encoding ASR forms a single operon with ASRT (ASR transducer) which is a $14 \mathrm{kDa}$ soluble protein, suggesting that ASR functions as a photochromic sensor by activating the soluble transducer. This article reviews the detailed photoreaction processes of ASR, which were studied by low-temperature Fourier-transform infrared (FTIR) and UV-visible spectroscopy. The former research reveals that the retinal isomerization is similar to bacteriorhodopsin (BR), but the hydrogen-bonding network around the Schiff base and cytoplasmic region is different. The latter study shows the stable photoproduct of the all-trans form is $100 \%$ 13-cis, and that of the 13-cis form is $100 \%$ all-trans. These results suggest that the structural changes of ASR in the cytoplasmic domain play important roles in the activation of the transducer protein, and photochromic reaction is optimized for its sensor function.
\end{abstract}

Keywords: photosensor; rhodopsin; photochromism; FTIR spectroscopy; UV-visible spectroscopy

\section{Introduction}

Photosynthesis is one of the most important chemical reactions in living cells because almost all energy spent by living things on Earth originates from it. Photosynthesis mainly takes place in the 
chloroplasts of plants, where a photoinduced electron transfer reaction first stores light energy, but eventually a proton gradient is formed across the membrane. The proton gradient is used for the synthesis of ATP, because it is a driving force of an enzyme ATP-synthase. Some bacteria directly convert light energy into a proton gradient through a proton pump. Light sensing is also important: Plants must avoid ultra-violet (UV) light, because it can possibly damage their genes. In addition, they have to sense orange or red light, as photosynthesis is more efficiency under these lights.

Four archaeal type rhodopsins [Bacteriorhodopsin (BR), Halorhodopsin (HR), Sensory rhodopsin I (SRI), and Sensory rhodopsin II (SRII); also called phoborhodopsin (pR)] were discovered in the cytoplasmic membrane of Halobacterium salinarum [1-4]. The former two (BR and HR) function as light-driven proton and chloride pumps, respectively, while the latter two (SRI and SRII) are responsible for attractive and repellent phototaxis, respectively (Figure 1). They have a retinal molecule as a chromophore, which forms a Schiff base linkage with a lysine residue of the 7th helix. An all-trans form (all-trans, 15-anti) is the functional form in BR, HR, SRI and SRII, and absorption of light leads to isomerization to the 13-cis, 15-anti form, which triggers protein structural changes for function. In the case of $\mathrm{BR}$, a cyclic reaction comprises the series of intermediates, $\mathrm{K}, \mathrm{L}, \mathrm{M}, \mathrm{N}$, and $\mathrm{O}$ (Figure 2) [5,6]. During the photocycle, a proton is transported from the cytoplasmic to the extracellular side. They have been extensively studied as model systems converting light energy to chemical potential or environmental signals. Although such archaeal type rhodopsins were considered to exist only in Archaea, during the last decade the various genome sequencing projects have revealed that archaeal rhodopsins also exist in Eukaryota and Bacteria. In eucaryotes, archaeal rhodopsins have been found in fungi [8], green algae [9,10], dinoflagellates [11], and cryptomonads [12].

Figure 1. The bacteriorhodopsin photocycle (PDB code: 1C3W) [7]. The reaction starts with light and returns to the initial state through the various intermediates within $10 \mathrm{~ms}$.

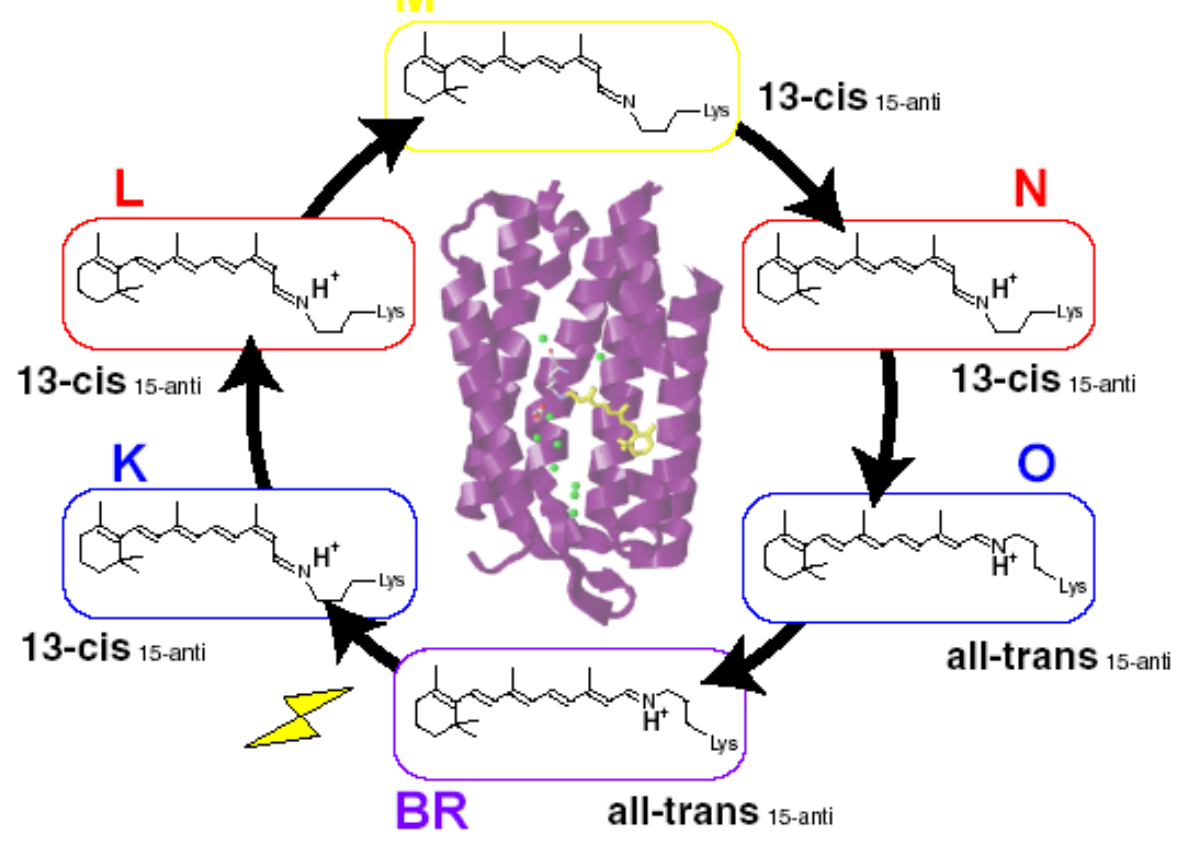


Figure 2. There are four archaeal rhodopsins in Halobacterium salinarum: bacteriorhodopsin, halorhodopsin, sensory rhodopsin I and II. All of them have seven transmembrane helices and an all-trans retinal as a chromophore.

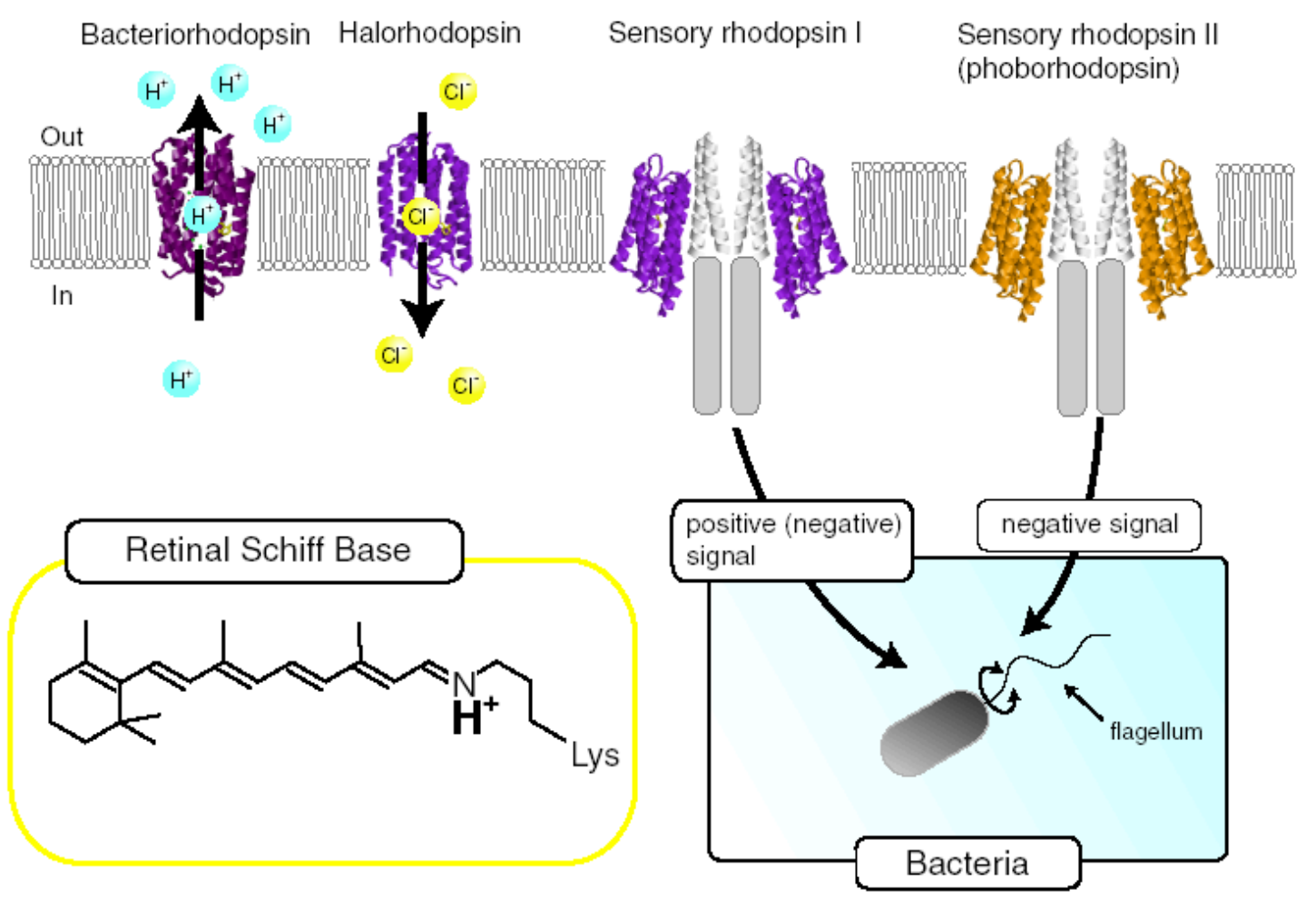

Eubacterial rhodopsins were found both in $\gamma$ - and $\alpha$-proteobacteria $[13,14]$ as well as in Anabaena (Notstoc) sp. PCC7120, a freshwater cyanobacterium [15], which was called Anabaena Sensory Rhodopsin (ASR). The gene encoding ASR, which is a membrane protein of 261 residues ( $26 \mathrm{kDa})$, and a smaller gene encoding a soluble protein of 125 residues $(14 \mathrm{kDa})$, which is called ASRT (ASR transducer), are under the same promoter in a single operon [15]. The opsin expressed heterologously in Escherichia coli membranes binds all-trans retinal to form a pink pigment $\left(\lambda_{\max }=549 \mathrm{~nm}\right)$ with a photochemical reaction cycle half-life of $110 \mathrm{~ms}\left(\mathrm{pH} 6.8,18{ }^{\circ} \mathrm{C}\right.$ ) [15]. The previous study revealed that co-expression with ASRT increased the rate of the photocycle, indicating physical interaction with ASR and the possibility that ASR works as a photosensor protein (Figure 3) [15]. It should be noted that SRI and SRII activate transmembrane transducer proteins (Figure 1). In this sense, ASR is closer to visual rhodopsins that activate soluble G-proteins.

According to the X-ray crystal structure of ASR (Figure 4), it is similar to those of other archaealtype rhodopsins. ASR accommodates both all-trans and 13-cis retinal in the ground state, which can be interconverted by illumination with blue $(480 \mathrm{~nm})$ or orange $(590 \mathrm{~nm})$ light (Figure 3) [16]. Such photochromic behavior has never been observed in other archaeal rhodopsins such as BR, HR, SRI and SRII, being characteristic to ASR. These results suggested that ASR could be a photochromic color sensor, whereas nothing was well-known about the structural changes and scheme of its photochromic reactions when we started the study. 
Figure 3. Proposed function of Anabaena sensory rhodopsin (1XIO[16]). ASR is interconverted into two isomeric states, which have different interactions with ASRT (2II9) [17]. ASRT maybe controls the expression level of phycobilisome proteins (phycocyanin and phycoerythrin) [15]. Phycobilisome graphic is from Grossman et al. [18]

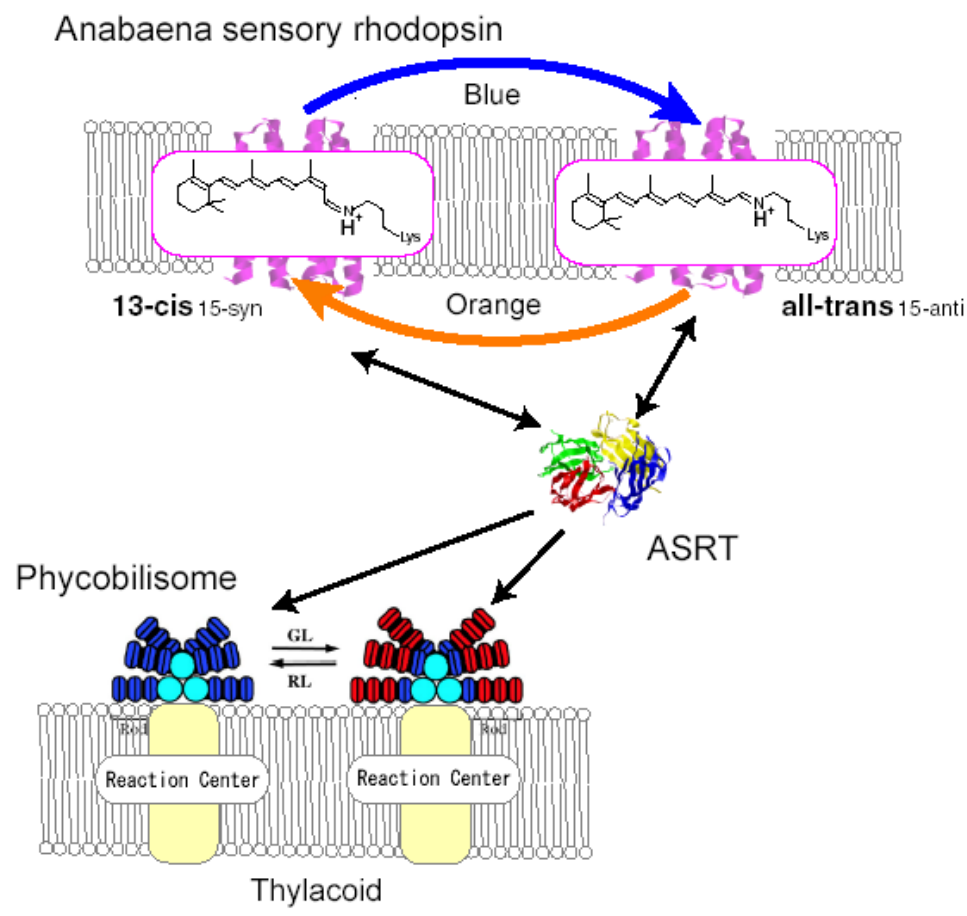

Figure 4. (Left) X-ray crystallographic structure of ASR (1XIO[16]). Purple ribbons, green spheres, yellow and white sticks correspond to helices, water molecules, retinal and amino acid residues, respectively. (Right) The Schiff base region of ASR and BR (translucent structure, 1C3W [7]), respectively. Each retinal molecule between ASR and BR is fitted to compare the hydrogen-bonding networks by using Swiss-PdbViewer [19]. Top and bottom regions correspond to the cytoplasmic and extracellular sides. The green sphere (Water 402) represents a water molecule which forms a hydrogen bond bridge between the protonated Schiff base and its counterion, Asp75. Hydrogen-bonds (blue dashed lines) are inferred from the structure and the numbers are the hydrogen-bond distances in $\AA$. This figure is adapted with permission from Furutani et al. [20]. Copyright 2005 American Chemical Society.

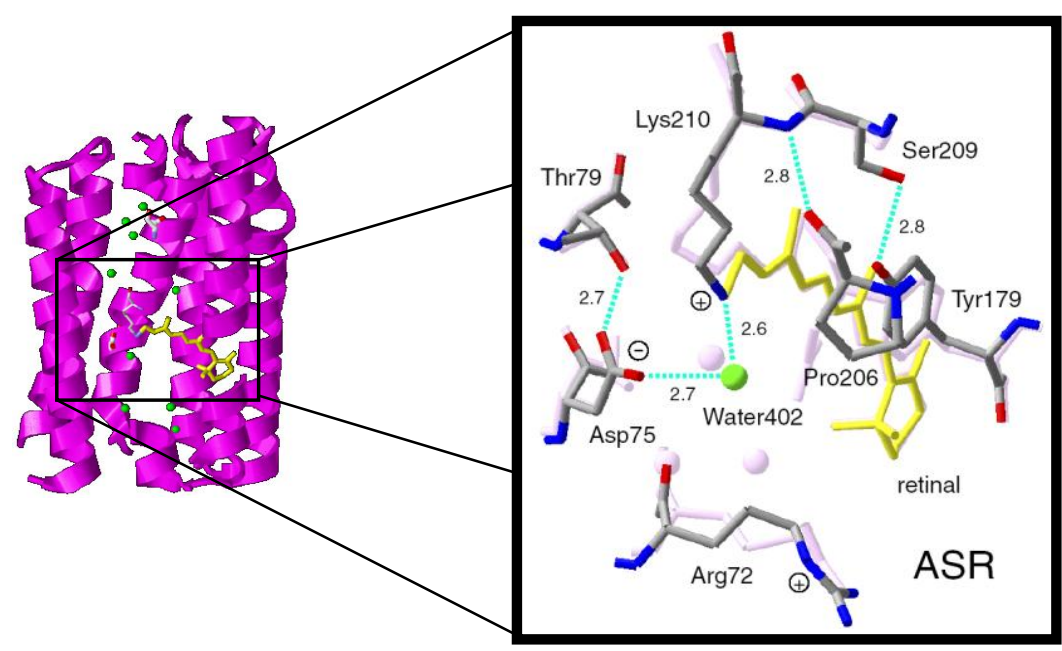


Amino acid sequence comparison between ASR and BR reveals that some important residues for pumping protons are replaced in ASR. The proton donor to the Schiff base (Asp96 in BR) and one of proton release groups (Glu194 in BR) are replaced by serine residues, Ser86 and Ser188, respectively (Figure 5).

Figure 5. Comparison of amino acid sequences of ASR and BR. The transmembrane topology is based on the crystallographic three-dimensional structures. The sequence alignment was done using CLUSTAL W [21] with the default settings. Single letters in a circle denote residues common to ASR and BR. The residues that are different in ASR and $\mathrm{BR}$ are denoted at the top and bottom of the circles, respectively. The residues forming the retinal binding site within $5 \AA$ of the chromophore are shown by bold or filled circles. This figure is reprinted with permission from Furutani et al. [20]. Copyright 2005 American Chemical Society.

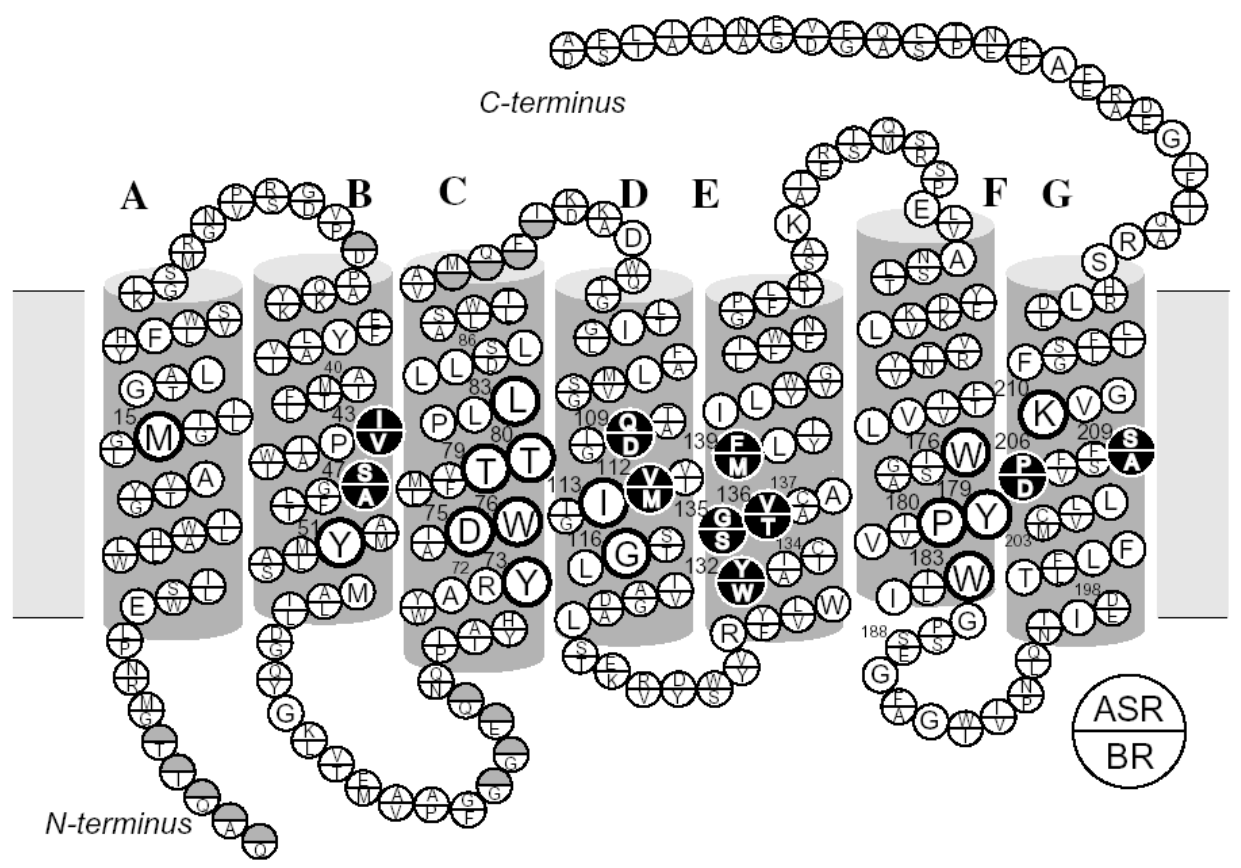

Ten amino acid residues out of twenty-five which constitute the retinal binding site are different from those of BR, probably accounting for the different absorption maximum and photochromic behavior of ASR. Among them, the most characteristic replacement is Pro206 located at the position of Asp212 in $\mathrm{BR}$, which is one of the counterions of the Schiff base and a well conserved amino acid residue in archaeal type rhodopsins (Figure 6). The influence of Pro206 on the hydrogen bonds around the Schiff base should be studied precisely for elucidating the difference in the structural changes of retinal and protein between ASR and BR upon their activation.

As shown above, ASR is a unique archaeal-type rhodopsin. However, the molecular properties, particularly the nature of its photochromic behavior, were much less known. Thus, in the last five years, we have studied various properties of ASR, mainly by use of spectroscopic techniques. Since ASR possesses visible absorption, UV-visible spectroscopy is the basic technique to study the properties of this molecule. Low-temperature UV-visible spectroscopy was used to study the photochromism of ASR described in Section 4. In the photochromic reaction, the isomeric states of the retinal chromophore 
play an important role, and HPLC analysis is the direct method to determine the isomeric composition of the retinal chromophore. We used this method in the work described in Section 3. On the other hand, in our studies we have mostly used low-temperature Fourier-transform infrared (FTIR) spectroscopy [22,23]. Infrared frequencies cover the 4,000-100 $\mathrm{cm}^{-1}$ region, which corresponds to the molecular vibrations of interest, so infrared spectroscopy is a particularly suitable experimental tool to study structural changes in proteins. In this review article, Sections 2 and 3 cover the local structural analysis of retinal photoisomerization $(77 \mathrm{~K}$ ) of the all-trans and 13-cis forms of ASR, respectively, while Section 5 describes cytoplasmic surface structural perturbation of all-trans ASR at $170 \mathrm{~K}$.

Figure 6. The 25 amino acid sequence of archaeal-type rhodopsin around retinal. First, second, third, fourth and fifth categories represent the families of bacteriorhodopsin, halorhodopsin, sensory rhodopsin I, sensory rhodopsin II and other archaeal-type rhodopsins, respectively. position $S$ : near the Schiff base, $P$ : near the polyene chain, $\beta$ : around the $\beta$-ionon ring.

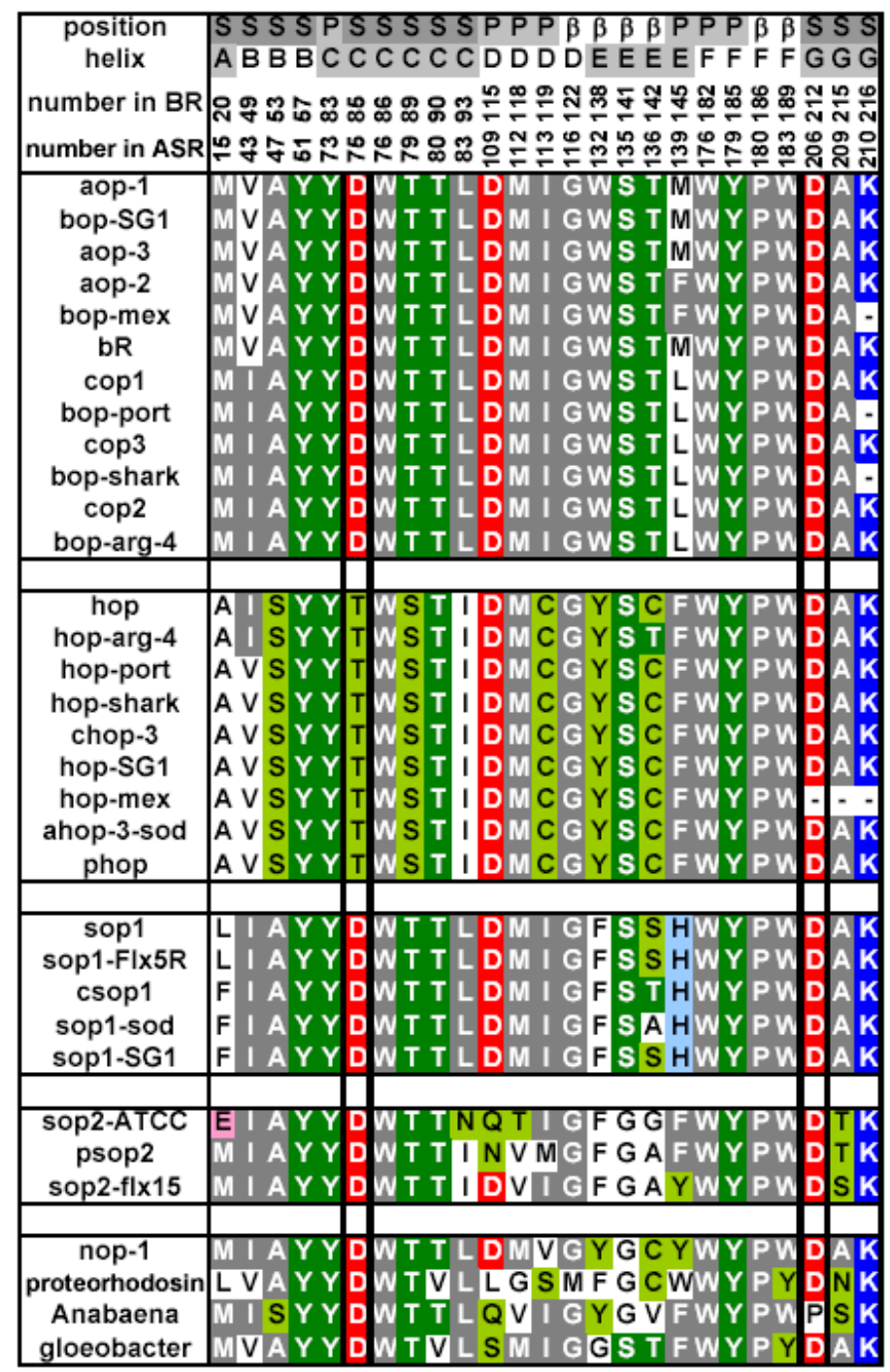




\section{FTIR Spectroscopy of the All-trans Form of Anabaena Sensory Rhodopsin at 77 K: Hydrogen Bond of a Water between the Schiff Base and Asp75}

As mentioned, comparison of the amino acid sequences of ASR and BR shows that some important residues for the proton pump in BR are replaced in ASR (Figure 5). The most characteristic replacement is Pro206 at the corresponding position of Asp212 in BR, Asp212 counterions a counterion complex of the Schiff base in BR, and the aspartate is highly conserved among archaeal rhodopsins. How is the hydrogen bonding network around the Schiff base modified in ASR by the presence of Pro206?

We have used low-temperature Fourier transform infrared (FTIR) spectroscopy to detect and study $\mathrm{X}-\mathrm{H}$ and $\mathrm{X}-\mathrm{D}(\mathrm{X}=\mathrm{O}, \mathrm{N})$ stretching vibrations in the mid-infrared region $\left(4,000-1,800 \mathrm{~cm}^{-1}\right)[22,23]$. These vibrations are direct indications of the hydrogen bonding network, including internal water molecules. In fact, comparison of the $\mathrm{K}$ intermediate $\left(\mathrm{BR}_{\mathrm{K}}\right)$ minus $\mathrm{BR}$ difference spectra between hydration with $\mathrm{D}_{2} \mathrm{O}$ and $\mathrm{D}_{2}{ }^{18} \mathrm{O}$ in the X-D stretching region $\left(2,700-1,800 \mathrm{~cm}^{-1}\right)$ enabled us to assign the O-D stretching vibrations of water molecules not only with a weak hydrogen bond (at $>2,500 \mathrm{~cm}^{-1}$ ) but also with a strong hydrogen bond (at $<2,400 \mathrm{~cm}^{-1}$ ). A mutation study showed that one of the O-D stretches $\left(2,171 \mathrm{~cm}^{-1}\right)$ originates from a bridging water molecule between the Schiff base and its counterion (Asp85) [25]. Hydration switch of the water plays an important role in the proton transfer reaction in BR [26]. In addition and interestingly, comprehensive studies of BR mutants and other rhodopsins have revealed that strongly hydrogen-bonded water molecules are only found in the proteins exhibiting proton pump activities [27]. This suggests that a strongly hydrogen bonded water molecule that bridges the Schiff base and its counterion is essential for proton pumping, but in terms of this idea, our FTIR study of ASR is intriguing, because ASR possesses a bridged water like BR [16], but does not pump protons [15].

Here, we applied low-temperature FTIR spectroscopy to the all-trans form of ASR, and compared the difference spectra at $77 \mathrm{~K}$ with those of BR. The $\mathrm{K}$ intermediate minus ASR difference spectra show that the retinal isomerizes from the all-trans to the distorted 13-cis form, like BR. The N-D stretching of the Schiff base was observed at 2,163(-) and 2,125(-) $\mathrm{cm}^{-1}$, while the O-D stretchings of water molecules were observed in the region $>2,500 \mathrm{~cm}^{-1}$. These results indicate that the protonated Schiff base forms a strong hydrogen bond with a water molecule, which is connected to Asp75 with a weak hydrogen bond. This result supports our working hypothesis about the strong correlation between the proton pump activity and the existence of strongly hydrogen bonded water molecules in archaeal rhodopsins. We shall discuss in some detail the structural reason why the bridged water molecule does not form a strong hydrogen bond in ASR.

In spectroscopic studies of archaeal rhodopsins, it is important to distinguish between the separate the photocyclization products of the all-trans from the 13-cis forms. In the case of BR, a well-known light adaptation procedure leads to a complete all-trans form. On the other hand, Vogeley et al. reported that ASR has a maximal amount of the all-trans form in the dark (>75\%), while light adaptation rather decreases the amount of the all-trans form [16]. This was reproduced in this study, and hence, we used the dark-adapted ASR sample. The absorption maximum of the all-trans enriched ASR was located at $549 \mathrm{~nm}$, which was the same value previously reported [16]. Low-temperature UV-visible spectroscopy of ASR showed that the red-shifted intermediate $\left(\mathrm{ASR}_{\mathrm{K}}\right)$ is formed at $77 \mathrm{~K}$. 
The difference absorption maximum was located at $593 \mathrm{~nm}$, and we estimated the absolute absorption maximum of $\mathrm{ASR}_{\mathrm{K}}$ at $589 \mathrm{~nm}$ (data not shown).

\subsection{Comparison of the Difference Infrared Spectra Obtained by the Photoreactions of Anabaena} Sensory Rhodopsin (ASR) at $77 \mathrm{~K}$ with Those of Bacteriorhodopsin (BR).

Figure 7 shows the $\mathrm{ASR}_{\mathrm{K}}$ minus ASR (a) and $\mathrm{BR}_{\mathrm{K}}$ minus BR spectra (b), which were measured at $77 \mathrm{~K}$ upon hydration with $\mathrm{H}_{2} \mathrm{O}$ (solid lines) and $\mathrm{D}_{2} \mathrm{O}$ (dotted lines). Unlike those of $\mathrm{BR}$, the difference spectra of ASR contain a mixture of photoproducts of the all-trans and 13-cis form. However, we estimated by use of the marker band $\left(1,178 \mathrm{~cm}^{-1}\right)$ that the 13 -cis form contribution under our illumination conditions is less than $20 \%$ (see below). Almost all vibrational bands described in this chapter originate from the photoreaction of the all-trans form.

Figure 7. The $\mathrm{ASR}_{\mathrm{K}}$ minus $\mathrm{ASR}$ (a) and the $\mathrm{BR}_{\mathrm{K}}$ minus $\mathrm{BR}$ (b) spectra in the $1,800-850 \mathrm{~cm}^{-1}$ region measured at $\mathrm{pH} 7$ and $77 \mathrm{~K}$ upon hydration with $\mathrm{H}_{2} \mathrm{O}$ (solid line) and $\mathrm{D}_{2} \mathrm{O}$ (dotted line), respectively. In the hydrated film, ASR molecules are oriented randomly, while BR molecules are highly oriented. Spectrum in (b) is reproduced from Kandori et al. [28], where the sample window is tilted by $53.5^{\circ}$. One division of the y-axis corresponds to 0.005 absorbance units. This figure is reprinted with permission from Furutani et al. [20]. Copyright 2005 American Chemical Society.

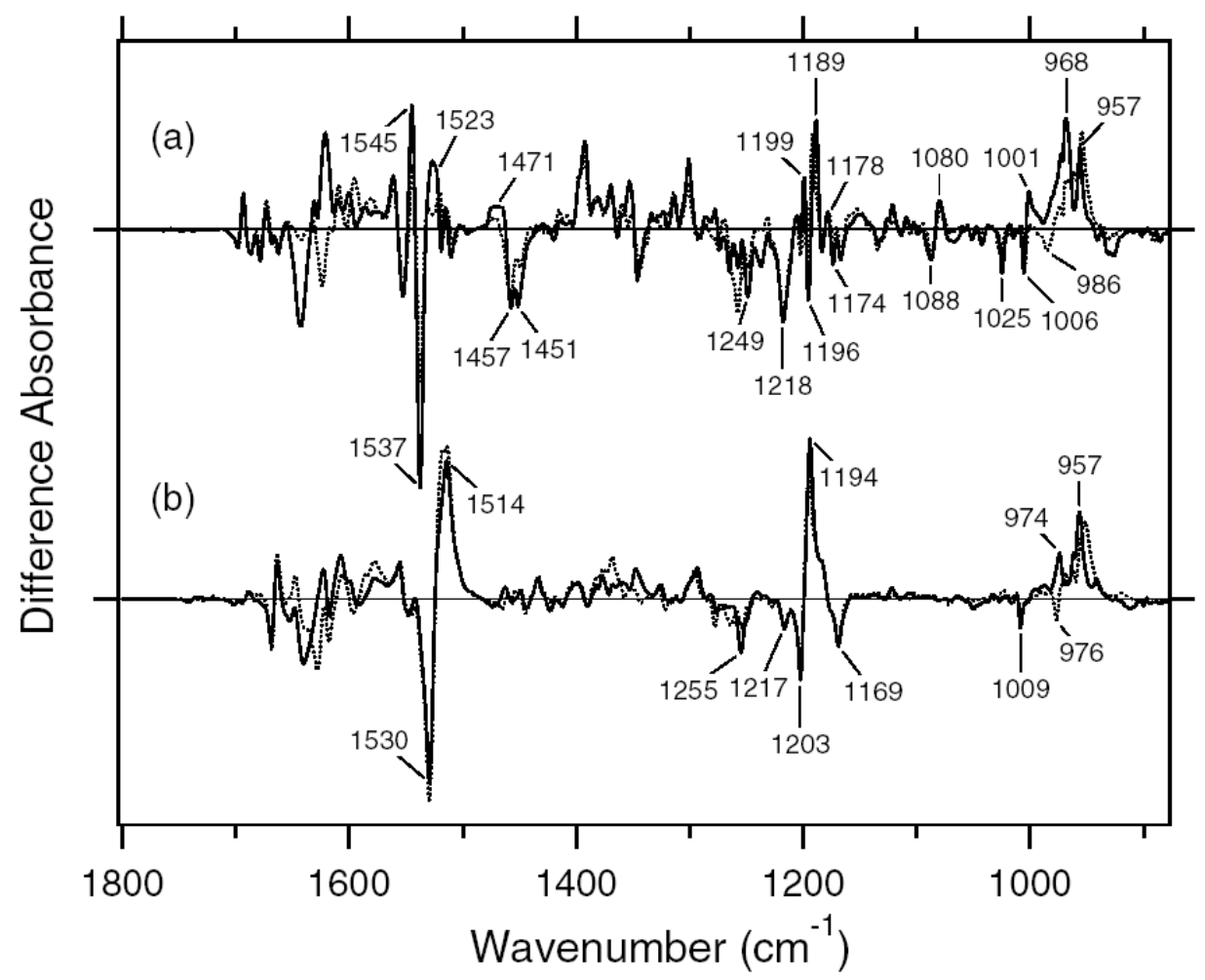

The negative band at $1,537 \mathrm{~cm}^{-1}$ corresponds to the ethylenic stretching vibration of the all-trans chromophore in ASR, which exhibits an absorption maximum at $549 \mathrm{~nm}$ [16]. The frequency is in good agreement with the well-known linear correlation between the ethylenic stretching frequencies 
and absorption maxima for various retinal proteins [29]. In the case of BR, the bands at $1,530(-) / 1,514(+) \mathrm{cm}^{-1}$ correspond to the ethylenic stretching vibrations of the unphotolyzed and $\mathrm{K}$ intermediate $\left(\mathrm{BR}_{\mathrm{K}}\right)$ states, respectively (Figure $7 \mathrm{~b}$ ). On the other hand, two positive bands appeared at 1,545 and $1,523 \mathrm{~cm}^{-1}$ for ASR (Figure 7a). According to the ethylenic stretching frequencies and absorption maxima correlation we predicted the ethylenic stretch of $\operatorname{ASR}_{\mathrm{K}}(589 \mathrm{~nm})$ to be at $1,525 \mathrm{~cm}^{-1}$. Therefore, the $1,523 \mathrm{~cm}^{-1}$ band is likely to be the latter, and the band at $1,545 \mathrm{~cm}^{-1}$ can possibly be assigned to the amide II mode. A similar observation was made for halorhodopsin [30], where the $\mathrm{K}$ intermediate exhibits two positive bands at 1,538 and $1,514 \mathrm{~cm}^{-1}$ with a negative band at $1,525 \mathrm{~cm}^{-1}$.

Remarkable spectral differences between ASR and BR were seen in the 1,500-1,450 $\mathrm{cm}^{-1}$ region. Two negative bands at 1,457 and $1,451 \mathrm{~cm}^{-1}$ and a positive band at $1,471 \mathrm{~cm}^{-1}$ were observed for ASR (Figure 7a). Among these three bands, the $1,457 \mathrm{~cm}^{-1}$ band is insensitive to H-D exchange, whereas the bands at 1,471 and $1,451 \mathrm{~cm}^{-1}$ are reduced to the half the intensity in $\mathrm{D}_{2} \mathrm{O}$. On the other hand, such strong bands are absent for BR (Figure 7b). This frequency region corresponds to the imide II vibrations of proline.

\subsection{Comparison of the Vibrational Bands of the Retinal Chromophore between ASR and BR}

The C-C stretching vibrations of retinal in the $1,290-1,100 \mathrm{~cm}^{-1}$ region are sensitive to the local structure of the chromophore. In Figure $8 b$, the negative bands at 1,217, 1,169, 1,254, and 1,203 $\mathrm{cm}^{-1}$ were assigned to the $\mathrm{C} 8-\mathrm{C} 9, \mathrm{C} 10-\mathrm{C} 11, \mathrm{C} 12-\mathrm{C} 13$, and $\mathrm{C} 14-\mathrm{C} 15$ stretching vibrations of $\mathrm{BR}$, respectively [31]. These frequencies are characteristic of the all-trans retinal protonated Schiff base, though the frequencies are higher because of the charge delocalization of the retinal molecule in BR. Upon formation of $\mathrm{BR}_{\mathrm{K}}$, retinal isomerizes to the 13-cis form, resulting in the appearance of a strong positive band at $1,194 \mathrm{~cm}^{-1}$, which is assigned to $\mathrm{C} 10-\mathrm{C} 11$ and $\mathrm{C} 14-\mathrm{C} 15$ stretching vibrations [32]. A more complex spectral feature was observed for ASR in the 1,290-1,100 $\mathrm{cm}^{-1}$ region (Figure 8a). One reason is that the photoreaction of the 13-cis form to its photoproduct contributes to these spectra. It is known that a positive band at $\sim 1,180 \mathrm{~cm}^{-1}$ is a marker band of such a reaction in BR [33]. Similarly, in this study for ASR, we found that the bands at $1,183(-) / 1,178(+) \mathrm{cm}^{-1}$ increase in intensity when illumination wavelengths are changed. Thus, we interpreted that these bands originate from the photoreaction of the 13-cis form in ASR as well as in BR. In other words, we established the illumination conditions to maximally reduce the bands at $1,183(-) / 1,178(+) \mathrm{cm}^{-1}$ in this study.

In the case of the all-trans form of ASR, the negative bands at 1,218, 1,174 (and/or 1,167), 1,249, and $1,196 \mathrm{~cm}^{-1}$ were tentatively assigned to the $\mathrm{C} 8-\mathrm{C} 9, \mathrm{C} 10-\mathrm{C} 11, \mathrm{C} 12-\mathrm{C} 13$, and $\mathrm{C} 14-\mathrm{C} 15$ stretching vibrations, respectively (Figure 8a). These frequencies are similar to those of BR (each frequency difference is $<10 \mathrm{~cm}^{-1}$ ), supporting the fact that the retinal configuration of ASR in the dark-adapted state is all-trans. However, the relatively large difference in C12-C13 $\left(6 \mathrm{~cm}^{-1}\right)$ and C14-C15 $\left(7 \mathrm{~cm}^{-1}\right)$ stretching vibrations suggests that the retinal structure near the Schiff base region is somehow different in ASR and BR. In addition, the intensity of the band at $1,218 \mathrm{~cm}^{-1}$ is three times larger than that of BR, which also suggests different retinal structure around the C8-C9 bond. Upon formation of $\mathrm{ASR}_{\mathrm{K}}$, the retinal molecule is considered to isomerize to the 13-cis form in analogy to the case of BR. However, unlike BR, there are three positive bands at $1,199,1,189$, and $1,149 \mathrm{~cm}^{-1}$. The $1,199 \mathrm{~cm}^{-1}$ band is not sensitive to H-D exchange, suggesting the origin as a C-C stretching vibration in the polyene chain of 
the retinal molecule. The bands at 1,189 and $1,149 \mathrm{~cm}^{-1}$ are upshifted upon hydration with $\mathrm{D}_{2} \mathrm{O}$, suggesting that they are influenced by the Schiff base vibration. The $1,189 \mathrm{~cm}^{-1}$ band can be assigned to the C14-C15 stretching vibration, while the $1,149 \mathrm{~cm}^{-1}$ band is difficult to identify at present. The downshift of the C14-C15 stretching vibration from 1,196 to $1,189 \mathrm{~cm}^{-1}$ upon formation of $\mathrm{ASR}_{\mathrm{K}}$ suggests that the retinal configuration is 13 -cis in $\mathrm{ASR}_{\mathrm{K}}$. Splitting into two negative bands at 1,174 and $1,167 \mathrm{~cm}^{-1}$ may suggest the presence of a positive band at $1,171 \mathrm{~cm}^{-1}$, which can be assigned to the C10-C11 stretching vibration.

Figure 8. The $\mathrm{ASR}_{\mathrm{K}}$ minus $\mathrm{ASR}$ (a) and the $\mathrm{BR}_{\mathrm{K}}$ minus $\mathrm{BR}$ (b) spectra in the $1,290-1,100 \mathrm{~cm}^{-1}$ region, which correspond to $\mathrm{C}-\mathrm{C}$ stretching vibrations and $\mathrm{N}-\mathrm{H}$ in-plane bending vibrations of the retinal chromophore. The sample was hydrated with $\mathrm{H}_{2} \mathrm{O}$ (solid lines) or $\mathrm{D}_{2} \mathrm{O}$ (dotted lines). One division of the $\mathrm{y}$-axis corresponds to 0.004 absorbance units. This figure is reprinted with permission from Furutani et al. [20]. Copyright 2005 American Chemical Society.

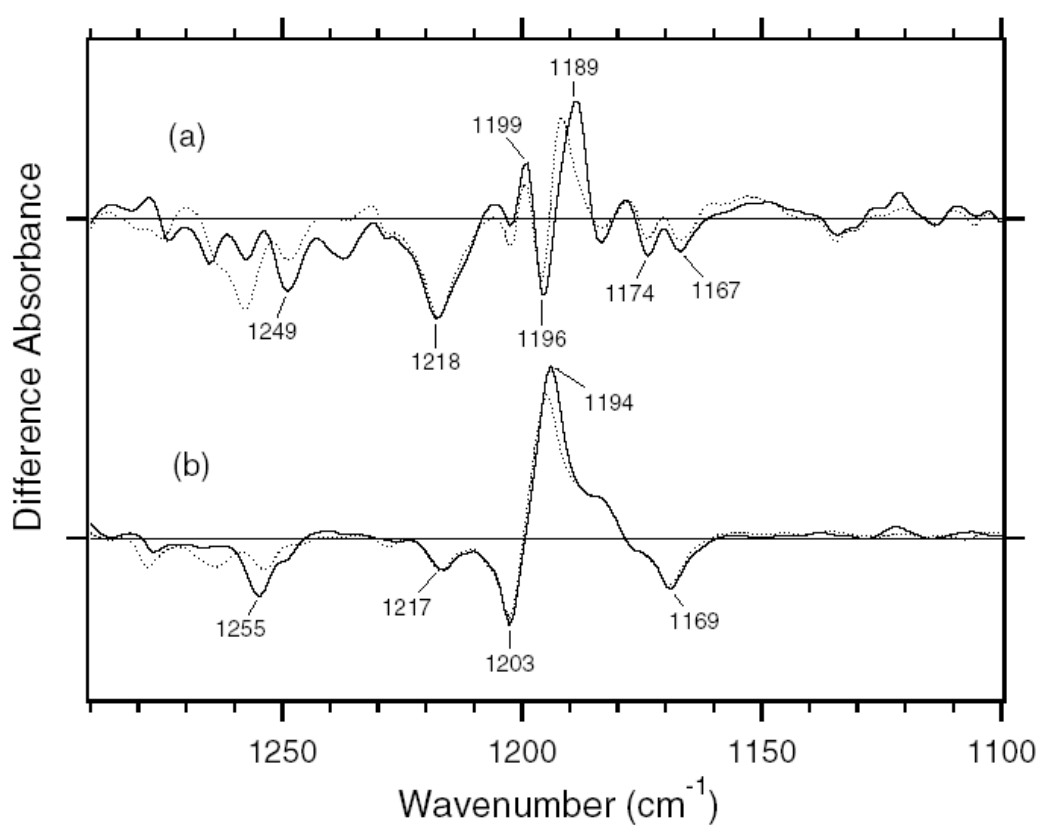

The H-D exchangeable band at $1,255 \mathrm{~cm}^{-1}$ was assigned to one of the modes containing the N-H in-plane bending vibration of the Schiff base of BR [34], while similar negative bands appear at $1,249 \mathrm{~cm}^{-1}$ in the spectra of ASR. The band disappearing upon hydration with $\mathrm{D}_{2} \mathrm{O}$ can be assigned to the modes of the Schiff base. The neighboring negative band at $1,237 \mathrm{~cm}^{-1}$ is also sensitive to deuteration and seen only in ASR, but its origin remains unknown. The result suggests that the hydrogen bonding environment of the Schiff base of ASR is similar to that of BR.

The difference spectra in the $1,110-890 \mathrm{~cm}^{-1}$ region are expanded in Figure 9. Hydrogen-out-of-plane (HOOP), N-D in-plane bending, and methyl rocking vibrations are observed here, and the presence of strong HOOP modes represents the distortion of the retinal molecule at the corresponding position. The most intense $\mathrm{HOOP}$ band in the $\mathrm{BR}_{\mathrm{K}}$ minus $\mathrm{BR}$ difference spectra (Figure 9b) was observed at $957 \mathrm{~cm}^{-1}$ (in $\mathrm{H}_{2} \mathrm{O}$ ) and $951 \mathrm{~cm}^{-1}$ (in $\mathrm{D}_{2} \mathrm{O}$ ), which were assigned to the C15-H HOOP vibration of $\mathrm{BR}_{\mathrm{K}}$ [34]. The origins of the bands at 941, 962, and $974 \mathrm{~cm}^{-1}$ remain 
unknown, but they may be assigned to HOOP vibrations. On the other hand, the weak negative band at $911 \mathrm{~cm}^{-1}$ was assigned to the $\mathrm{C} 15-\mathrm{H}$ and N-H HOOP vibrations of the original state of BR [35]. These results have been interpreted as an increase in the retinal distortion around the Schiff base upon the retinal isomerization in BR. In the case of ASR, similar but slightly upshifted bands were observed. The positive bands at 1,001, 973, 968, and $957 \mathrm{~cm}^{-1}$ of $\mathrm{ASR}_{\mathrm{K}}$ (Figure 9a) possibly correspond to those at 974, 962, 957, and $941 \mathrm{~cm}^{-1}$ of $\mathrm{BR}_{\mathrm{K}}$, respectively (Figure 9b). The negative bands at 932 and $927 \mathrm{~cm}^{-1}$ have probably the same origin as that at $911 \mathrm{~cm}^{-1}$ in $\mathrm{BR}$, which was assigned to the $\mathrm{C} 15-\mathrm{H}$ and N-H HOOP vibrations [35].

The negative band at $976 \mathrm{~cm}^{-1}$ and the positive band at $969 \mathrm{~cm}^{-1}$ in Figure $9 \mathrm{~b}$ were assigned to the $\mathrm{N}-\mathrm{D}$ in-plane bending vibrations of $\mathrm{BR}$ and $\mathrm{BR}_{\mathrm{K}}$, respectively [34]. The $1,009 \mathrm{~cm}^{-1}$ band is insensitive to $\mathrm{H}-\mathrm{D}$ exchange and was assigned to the methyl rocking vibration of the retinal in BR. The band at $1,006 \mathrm{~cm}^{-1}$ in Figure $9 \mathrm{a}$ can also be assigned to the methyl rocking vibration in ASR similarly. On the other hand, the bands at 1,088(-), 1,080(+), and 1,025(-) $\mathrm{cm}^{-1}$ are highly characteristic of the $\mathrm{ASR}_{\mathrm{K}}$ minus ASR difference spectra, and never observed in other archaeal-type rhodopsins such as BR, $p \mathrm{pR}$, and NR [34,36,37]. According to the literature, the antisymmetric $\mathrm{NC}_{3}$ stretchings of tertiary amines appear in the 1,250-1,000 $\mathrm{cm}^{-1}$ region [38]. Thus, these bands may originate from the skeletal vibrations of Pro206 as well as those at $1,471(+), 1,457(-)$, and $1,451(-) \mathrm{cm}^{-1}$ (Figure $7 \mathrm{a}$ ).

Figure 9. The $\mathrm{ASR}_{\mathrm{K}}$ minus $\mathrm{ASR}$ (a) and the $\mathrm{BR}_{\mathrm{K}}$ minus $\mathrm{BR}$ (b) spectra in the $1,110-890 \mathrm{~cm}^{-1}$ region, which correspond to hydrogen-out-of-plane (HOOP) vibrations of the retinal chromophore. The sample was hydrated with $\mathrm{H}_{2} \mathrm{O}$ (solid lines) or $\mathrm{D}_{2} \mathrm{O}$ (dotted lines). One division of the y-axis corresponds to 0.002 absorbance units. This figure is reprinted with permission from Furutani et al. [20]. Copyright 2005 American Chemical Society.

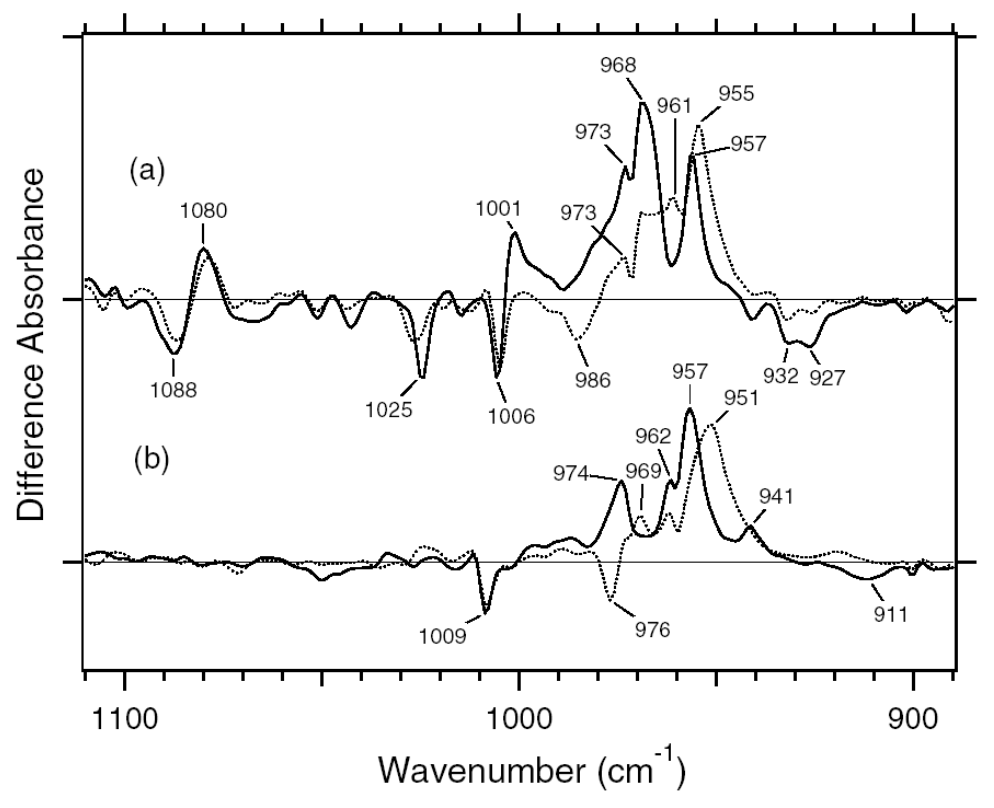

$\mathrm{C}=\mathrm{N}$ stretching vibrations of the protonated retinal Schiff base are observed in the $1,650-1,600 \mathrm{~cm}^{-1}$ region (Figure 10). The $\mathrm{C}=\mathrm{N}$ stretching vibrations are sensitive to $\mathrm{H}-\mathrm{D}$ exchange, and the difference in frequency has been considered as the probe for its hydrogen bonding strength $[39,40]$, that is, the larger 
the difference is, the stronger the hydrogen bond is. The $\mathrm{C}=\mathrm{NH}$ and $\mathrm{C}=\mathrm{ND}$ stretching vibrations of $\mathrm{BR}$ were observed at 1,641 and $1,628 \mathrm{~cm}^{-1}$, while those of $\mathrm{BR}_{\mathrm{K}}$ were at 1,608 and $1,606 \mathrm{~cm}^{-1}$, respectively [41]. The former difference in frequency is $13 \mathrm{~cm}^{-1}$, and the latter is $2 \mathrm{~cm}^{-1}$, suggesting that the protonated Schiff base forms a hydrogen bond in BR that is broken upon retinal isomerization. The $\mathrm{C}=\mathrm{N}$ stretches were observed at 1,642 $(\mathrm{C}=\mathrm{NH})$ and $1,624 \mathrm{~cm}^{-1}(\mathrm{C}=\mathrm{ND})$ in ASR, and its difference is $18 \mathrm{~cm}^{-1}$, suggesting that the hydrogen bonding strength is stronger than that of BR. On the other hand, it is difficult to assign the positive bands because of the more complicated spectral features. There are two sets of candidates for the $\mathrm{C}=\mathrm{N}$ stretching vibrations of $\mathrm{ASR}_{\mathrm{K}}$. One set is the bands at 1,621 $(\mathrm{C}=\mathrm{NH})$ and $1,610 \mathrm{~cm}^{-1}(\mathrm{C}=\mathrm{ND})$, while another set is the bands at $1,600(\mathrm{C}=\mathrm{NH})$ and $1,595 \mathrm{~cm}^{-1}(\mathrm{C}=\mathrm{ND})$. The differences in frequency are 11 and $5 \mathrm{~cm}^{-1}$ for the former and latter, respectively. If the former is the case, the hydrogen bond may not be broken upon retinal isomerization in ASR. Conclusive assignment of the $\mathrm{C}=\mathrm{N}$ stretching of $\mathrm{ASR}_{\mathrm{K}}$ needs stable isotope labeling on the Schiff base, which is shown in next chapter. On the other hand, the $\mathrm{N}-\mathrm{D}$ stretching vibration of the Schiff base in $\mathrm{D}_{2} \mathrm{O}$ provides more direct information about the hydrogen bond of the Schiff base, as described below.

Figure 10. The $\mathrm{ASR}_{\mathrm{K}}$ minus $\mathrm{ASR}$ (a) and the $\mathrm{BR}_{\mathrm{K}}$ minus $\mathrm{BR}$ (b) spectra in the $1,760-1,570 \mathrm{~cm}^{-1}$ region, most of which are ascribable for vibrations of the protein moiety. The underlined peaks are $\mathrm{C}=\mathrm{N}$ stretching vibrations of the chromophore. The sample was hydrated with $\mathrm{H}_{2} \mathrm{O}$ (solid lines) or $\mathrm{D}_{2} \mathrm{O}$ (dotted lines). One division of the $\mathrm{y}$-axis corresponds to 0.003 absorbance units. This figure is reprinted with permission from Furutani et al. [20]. Copyright 2005 American Chemical Society.

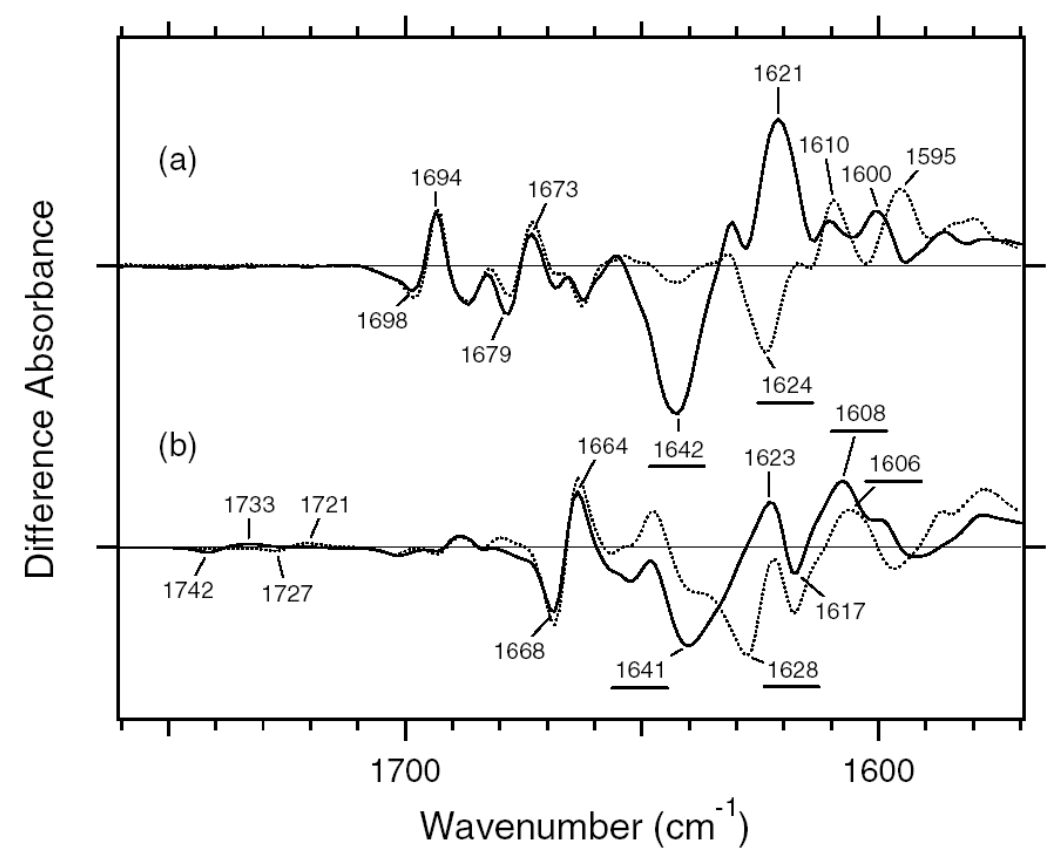

2.3. Comparison of the $C=O$ Stretching Vibrations of Carboxylate, Carbonyl, and Amide Groups of the Protein Moiety between ASR and BR

In the $\mathrm{BR}_{\mathrm{K}}$ minus $\mathrm{BR}$ difference spectra (Figure 10b), the bands at 1,742 and 1,733 $\mathrm{cm}^{-1}$ were assigned to the $\mathrm{C}=\mathrm{O}$ stretching vibrations of the protonated Asp115, which are downshifted upon 
hydration with $\mathrm{D}_{2} \mathrm{O}$ [42]. In contrast, there is no band in the same frequency region of the ASR spectra (Figure 10a), implying that Asp and Glu residues are located far from the retinal molecule even if they are protonated. ASR has a glutamine residue at the corresponding position of Asp115 in BR, whose vibrational bands are probably observed at 1,698(-) and 1,694(+) $\mathrm{cm}^{-1}$ (Figure 10a). Similar bands were also observed at $1,704(-)$ and $1,700(+) \mathrm{cm}^{-1}$ in the difference spectra of $p \mathrm{pR}$, which has an asparagine residue at the corresponding position [43]. These observations suggest that the structural changes around Asp115 in BR are similar among ASR, BR, and $p \mathrm{pR}$.

The band pairs at $1,668(-) / 1,664(+) \mathrm{cm}^{-1}$ and at $1,623(+) / 1,617(-) \mathrm{cm}^{-1}$ were assigned to the amide I $\mathrm{C}=\mathrm{O}$ stretching vibrations. The former was assigned to the amide I of $\alpha_{\text {II }}$ helix [44] and the latter to the amide I of Val49 [45]. In the case of ASR, a band pair at $1,679(-) / 1,673(+) \mathrm{cm}^{-1}$ could be similar in origin to the bands at $1,668(-) / 1,664(+) \mathrm{cm}^{-1}$ in BR. It should be noted that the spectral changes of amide I vibrations at $<1,660 \mathrm{~cm}^{-1}$ are much smaller in ASR than in BR, which is clearly seen in $\mathrm{D}_{2} \mathrm{O}$. This suggests that the structural changes of the peptide backbone in ASR upon retinal isomerization are very small. On the other hand, the structural perturbation of Pro206 was suggested for ASR.

\subsection{Comparison of the X-D Stretching Vibrations between ASR and BR}

X-D stretching vibrations of protein and water molecules appear in the 2,750-1,950 $\mathrm{cm}^{-1}$ region (Figure 11). A spectral comparison between the samples hydrated with $\mathrm{D}_{2} \mathrm{O}$ and $\mathrm{D}_{2}{ }^{18} \mathrm{O}$ identifies O-D stretching vibrations of water molecules which change their frequencies upon retinal photoisomerization. Green-labeled bands in Figure 11 can be assigned to the O-D stretching vibrations of water because of the isotope shift. In BR, six negative peaks at 2,690, 2,636, 2,599, 2,321, 2,292, and 2,171 $\mathrm{cm}^{-1}$ were earlier assigned to vibrations of water molecules (Figure 11b) [26,46]. The bands are widely distributed over the possible frequency range for stretching vibrations of water. Since the frequencies of the negative peaks at 2,321,2,292, and $2,171 \mathrm{~cm}^{-1}$ are much lower than those of fully hydrated tetrahedral water molecules [46], the hydrogen bonds of those water molecules must be very strong, possibly indicating their association with negative charges. Indeed, we assigned the $2,171 \mathrm{~cm}^{-1}$ band to the O-D group of a water molecule associated with deprotonated Asp85 [25]. This water molecule, called water 402 in the crystal structure of BR (PDB entry 1C3W), is located between the Schiff base and Asp85 (Figure 4). A previous QM/MM calculation of the Schiff base region of BR also supported the existence of an extremely strong hydrogen bond between water 402 and Asp85 [47]. Water stretching vibrations of $\mathrm{BR}_{\mathrm{K}}$ tend to be higher in frequency, implying that the overall hydrogen bonding becomes weaker upon photoisomerization.

In contrast, interestingly only three negative peaks at 2,690,2,640, and 2,608 $\mathrm{cm}^{-1}$ could be assigned to the O-D stretching vibrations of water in ASR (Figure 11a). The bands at 2,701, 2,653, and $2,573 \mathrm{~cm}^{-1}$ were assigned as water stretching vibrations of $\mathrm{ASR}_{\mathrm{K}}$. It should be emphasized that there are no water bands in the $<2,400 \mathrm{~cm}^{-1}$ region, which is a significant difference from the published results for $\mathrm{BR}$ and $p \mathrm{pR}$. In the case of $p \mathrm{pR}$, two pairs of peaks were observed in the $<2,400 \mathrm{~cm}^{-1}$ region, located at 2,369(+)/2,307(-) $\mathrm{cm}^{-1}$ and at 2,274(+)/2,215(-) $\mathrm{cm}^{-1}$ [47]. Since ASR has a bridged water molecule between the Schiff base and Asp75 (Figure 4) as well as BR and $p$ pR, one may expect similar 
water bands at $<2,400 \mathrm{~cm}^{-1}$. However, that is not the case for ASR. We will discuss the structural reason for the lack of strongly hydrogen bonded water molecules below.

The frequency region shown in Figure 11 also contains X-D stretching vibrations other than water molecules. In the $\mathrm{BR}_{\mathrm{K}}$ minus $\mathrm{BR}$ spectrum, the bands at 2,507(-)/2,466(+) $\mathrm{cm}^{-1}$ labeled in purple and the underlined bands at 2,466(+), 2,171(-), and 2,124(-) $\mathrm{cm}^{-1}$ were assigned to the O-D stretching vibrations of Thr89 [48,49] (the corresponding residue in ASR is Thr79) and the N-D stretching vibrations of the retinal Schiff base [41], respectively. Thus, the negative $2,171 \mathrm{~cm}^{-1}$ band contains both the O-D stretch of water and the N-D stretch of the Schiff base. In the ASR $_{K}$ minus ASR spectrum, there are 10 bands other than water bands: 2,547(-), 2,537(+), 2,508(-), 2,470(-), 2,446(+), 2,383(+), $2,336(-), 2,258(-), 2,163(-)$, and $2,125(-) \mathrm{cm}^{-1}$. The bands at 2,547(-)/2,537(+) $\mathrm{cm}^{-1}$ are attributed to the H-D unexchangeable S-H stretching vibration of a cysteine residue as described below. The bands at 2,508(-)/2,470(+) $\mathrm{cm}^{-1}$ can be assigned to the O-D stretching vibrations of Thr79 in analogy to BR. The O-D frequencies of Thr79 in ASR and $\operatorname{ASR}_{\mathrm{K}}\left(2,508\right.$ and 2,470 $\left.\mathrm{cm}^{-1}\right)$ are almost identical to those of Thr89 in BR and $\mathrm{BR}_{\mathrm{K}}\left(2,507\right.$ and 2,466 cm $\left.\mathrm{cm}^{-1}\right)$, respectively, indicating that the strength of hydrogen bonding between Thr79 and Asp75 is identical to that between Thr89 and Asp85 in BR.

Figure 11. Comparison of the difference infrared spectra of ASR (a) and BR (b) hydrated with $\mathrm{D}_{2} \mathrm{O}$ (red lines) or $\mathrm{D}_{2}{ }^{18} \mathrm{O}$ (blue lines) in the $2,730-1,950 \mathrm{~cm}^{-1}$ region. Green-labeled frequencies correspond to those identified as water stretching vibrations. Purple-labeled frequencies are O-D stretches of Thr89 [48,49], while the underlined frequencies are N-D stretches of the Schiff base [41]. Spectrum in (b) is reproduced from Tanimoto et al. [26], where the sample window is tilted by $53.5^{\circ}$. One division of the y-axis corresponds to 0.0007 absorbance units. This figure is reprinted with permission from Furutani et al. [20]. Copyright 2005 American Chemical Society.

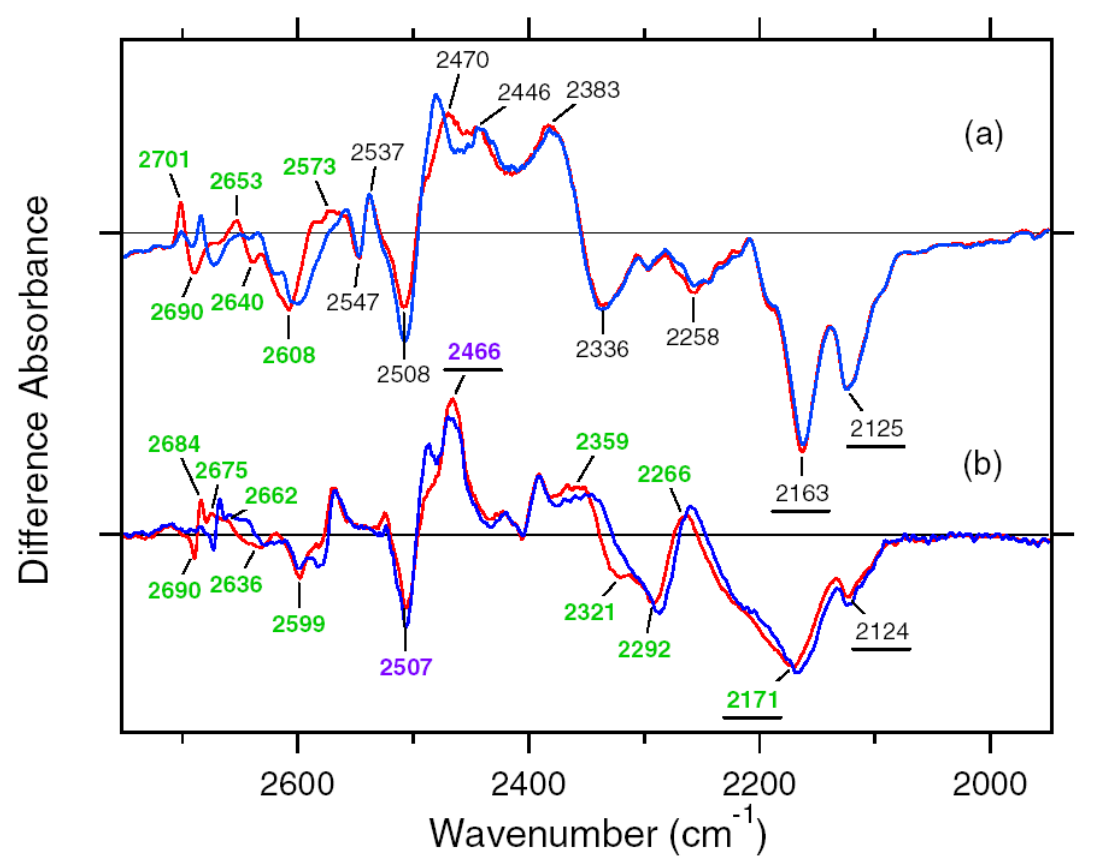

Though not assigned directly by use of the labeled protein, the bands at 2,163 and $2,125 \mathrm{~cm}^{-1}$ are likely to originate from N-D stretching of the Schiff base, whose frequencies are very similar to those in 
BR (2,171 and 2,124 $\left.\mathrm{cm}^{-1}\right)$. This fact indicates similar hydrogen bonding strengths between ASR and BR. The slightly lower frequency of the strong band $\left(2,163 \mathrm{~cm}^{-1}\right.$ in ASR and $2,171 \mathrm{~cm}^{-1}$ in BR) may correspond to the results obtained for the $\mathrm{C}=\mathrm{N}$ stretching vibrations as shown before (Figure 10). The analysis of the $\mathrm{C}=\mathrm{N}$ stretching vibrations of $\mathrm{ASR}_{\mathrm{K}}$ suggested two possibilities for the hydrogen bonding strength of the Schiff base. Figure 10a clearly shows the presence of the negative bands at 2,163 and 2,125 $\mathrm{cm}^{-1}$, implying that the N-D stretch is upshifted in $\mathrm{ASR}_{\mathrm{K}}$. We infer that one of the bands at $2,470,2,446$, and $2,383 \mathrm{~cm}^{-1}$ can be assigned to the N-D stretch in $\mathrm{ASR}_{\mathrm{K}}$. Thus, we can safely conclude that the hydrogen bond of the Schiff base in ASR becomes much weaker upon retinal photoisomerization as well as in BR.

\subsection{S-H Stretching Vibrations of the Cysteine Residues}

Figure 12 shows the $\mathrm{ASR}_{\mathrm{K}}$ minus ASR spectra in the 2,590-2,500 $\mathrm{cm}^{-1}$ (top panel) and $1,890-1,800 \mathrm{~cm}^{-1}$ (bottom panel) regions, which correspond to $\mathrm{S}-\mathrm{H}$ and $\mathrm{S}-\mathrm{D}$ stretching vibrations of cysteine residues, respectively. There are a negative band at $2,547 \mathrm{~cm}^{-1}$ and a positive band at $2,538 \mathrm{~cm}^{-1}$, while no band is observed in the S-D stretching upon hydration with $\mathrm{D}_{2} \mathrm{O}$.

Figure 12. The $\mathrm{ASR}_{\mathrm{K}}$ minus ASR spectra in the 2,590-2,500 $\mathrm{cm}^{-1}$ (the upper panel) and $1,890-1,800 \mathrm{~cm}^{-1}$ (the lower panel) region, which correspond to $\mathrm{S}-\mathrm{H}$ and $\mathrm{S}-\mathrm{D}$ stretching vibrations of cysteine residues, respectively. The sample was hydrated with $\mathrm{H}_{2} \mathrm{O}$ (solid lines) or $\mathrm{D}_{2} \mathrm{O}$ (dotted lines). One division of the $\mathrm{y}$-axis corresponds to 0.0001 absorbance units. This figure is reprinted with permission from Furutani et al. [20]. Copyright 2005 American Chemical Society.

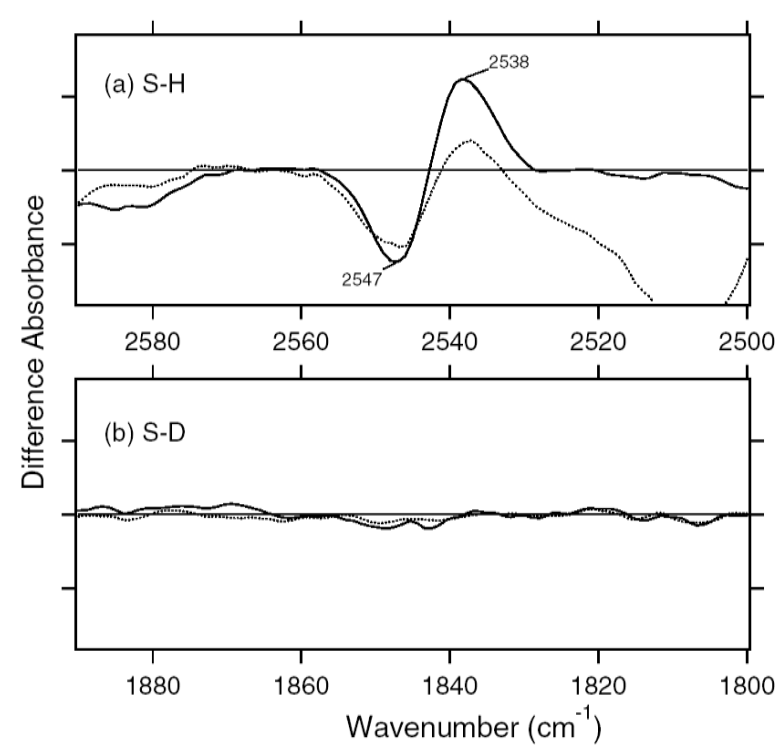

In fact, $\mathrm{S}-\mathrm{H}$ stretching vibrations in $\mathrm{D}_{2} \mathrm{O}$ are observed in Figure $11 \mathrm{a}\left(2,547\right.$ and $\left.2,537 \mathrm{~cm}^{-1}\right)$. The S-H stretching frequency of cysteine appears in the 2,580-2,525 $\mathrm{cm}^{-1}$ region. Thus, the frequency change from 2,547 to $2,538 \mathrm{~cm}^{-1}$ suggests that the cysteine forms a considerably strong hydrogen bond upon retinal isomerization. The non $\mathrm{H}$-D-exchangeable nature of the cysteine $\mathrm{S}-\mathrm{H}$ group presumably results from either the hydrophobic environment or the strong hydrogen bond. 
The lower-frequency shift in ASR is the opposite of the cysteine signal in the $\mathrm{NR}_{\mathrm{K}}$ minus NR spectra [37]. In addition, the H-D exchange is different between ASR and NR. These facts suggest that the cysteine residues are located in different environments and their hydrogen bonds change differently. There are three cysteine residues in ASR, Cys134 and Cys137 in helix E and Cys203 in helix G. Not all of them are conserved in archaeal-type rhodopsin, but Cys134 and Cys137 are located at a position similar to that of Cys170 in NR, which is conserved in halorhodopsin. The X-ray crystal structure of ASR also revealed that only the S-H group of Cys203 is directed to the inside of the protein. From these results, the observed band can be assigned to the S-H stretching of Cys203.

\subsection{Unique Structure of the All-trans Form of ASR}

In this study, we measured the $\mathrm{ASR}_{\mathrm{K}}$ minus ASR spectra by means of low-temperature FTIR spectroscopy. For this purpose, ASR was expressed in E. coli, and the wild-type protein was reconstituted into PC liposomes. It is noted that the ASR molecule is not embedded in the native membrane, which could modify the FTIR spectra. For instance, H-D exchange could be different between PC liposomes and the native membrane, a fact that should be elucidated in the future. However, this study focuses on the structural changes near the retinal upon photoisomerization, and the light-induced difference FTIR spectra are not significantly affected by different lipid environments.

Despite the presence of the 13-cis form, the obtained spectra are predominantly due to the photoreaction of the all-trans form, and the spectra were compared with those of BR. These results clearly show that the all-trans to 13-cis photoisomerization takes place in ASR like in BR, though the C-C stretching and HOOP vibrations are somehow different. The protonated Schiff base forms a strong hydrogen bond in ASR, presumably with the bridged water (Figure 4), and the hydrogen bond is cleaved by the rotation of the N-H (N-D) group, as in BR. We also observed S-H stretches of a cysteine residue which is insensitive to hydration with $\mathrm{D}_{2} \mathrm{O}$. We observed small amide $\mathrm{I}$ bands and large bands that can be ascribed to imide II $\left[1,471(+), 1,457(-)\right.$, and $\left.1,451(-) \mathrm{cm}^{-1}\right]$ and $\mathrm{NC}_{3}[1,088(-)$ and $\left.1,080(+) \mathrm{cm}^{-1}\right]$ stretchings of proline residues. A previous resonance Raman spectroscopic study showed that the imide II vibration of the X-Pro bond appears at around $1,450 \mathrm{~cm}^{-1}$ [50]. BR has three Pro residues in the transmembrane region, Pro50, Pro91, and Pro186 (Figure 5). The previous FTIR study suggested that the environment around these proline residues changes upon retinal isomerization via observation of the isotope effect of $\left[{ }^{15} \mathrm{~N}\right]$ proline in the $1,450-1,420 \mathrm{~cm}^{-1}$ region [51]. It should be noted that spectral changes are much smaller in BR than in ASR in this frequency region. In the case of ASR, there are additional three Pro residues (Figure 5). It is Pro206, a corresponding residue of Asp212 in BR (Figure 4). Figure 4 shows that the peptide $\mathrm{C}=\mathrm{O}$ group of Pro206 forms a hydrogen bond with the peptide amide (N-H group) of Lys210, which connects to a retinal chromophore. Thus, retinal photoisomerization strongly perturbs the peptide C-N bond of Pro206 in ASR, presumably leading to the appearance of these unusually intense bands in the $1,500-1,450 \mathrm{~cm}^{-1}$ region. It should be noted, however, that we can conclude this argument only when these bands are assigned by use of $\left[{ }^{15} \mathrm{~N}\right]$ proline-labeled ASR.

A significant difference between ASR and BR was seen for water bands. We have so far observed the O-D stretching vibrations of water molecules under strongly hydrogen bonded conditions in the $\mathrm{BR}_{\mathrm{K}}$ minus $\mathrm{BR}$ and $p \mathrm{pR}_{\mathrm{K}}$ minus $p \mathrm{pR}$ difference spectra $[26,36,46]$. The $\mathrm{X}$-ray crystal structures of $\mathrm{BR}$ and 
$p \mathrm{pR}$ reported the presence of a bridged water molecule between the Schiff base and its counterion (Asp85 in BR and Asp75 in $p \mathrm{pR}$ ) [7,52,53]. Therefore, the hydrogen bond of the water is expected to be strong, and such strongly hydrogen bonded water molecules were observed in the FTIR studies. The water molecules possess O-D stretches at 2,400-2,100 $\mathrm{cm}^{-1}$ in $\mathrm{D}_{2} \mathrm{O}[23,27]$. Since ASR has a bridged water molecule between the Schiff base and Asp75 (Figure 4) as well as BR and $p$ pR, one may expect similar water bands at $<2,400 \mathrm{~cm}^{-1}$. However, that was not the case for ASR. Therefore, the structural reason for the lack of strongly hydrogen bonded water molecules has to be explained on the basis of the structural background. Since both structures of ASR and BR are known (Figure 4), we will try to explain the reason here.

Our analysis of the Schiff base mode $(\mathrm{C}=\mathrm{N}$ stretch and $\mathrm{N}-\mathrm{D}$ stretch) in ASR showed that the hydrogen bonding strength of the Schiff base is similar in ASR and BR. This observation is consistent with the similar distance between the Schiff base nitrogen and water oxygen $(2.6 \AA$ for ASR and $2.9 \AA$ for BR). A slightly stronger hydrogen bond in ASR than in BR is also consistent with the distance that is shorter in ASR. In contrast, water bands in ASR were entirely different from those in BR, although the distance between the water oxygen and the oxygen of the counterion are similar $(2.7 \AA$ for ASR and $2.6 \AA$ for BR). The O-D stretch of the bridged water in BR is located at $2,171 \mathrm{~cm}^{-1}$ (Figure $11 \mathrm{~b}$ ), whereas that in ASR is probably one of the bands at 2,690, 2,640, and 2,608 $\mathrm{cm}^{-1}$ (Figure 11a). How is such difference observed between ASR and BR? It may be explained by the difference in the geometry of the hydrogen bond. Figure 13 shows that the $\mathrm{N}-\mathrm{O}_{\text {water }}-\mathrm{O}_{\text {Asp75 }}$ (the Schiff base nitrogen, the water oxygen, and the oxygen of Asp75, respectively) angle in ASR is $83^{\circ}$. The corresponding $\mathrm{N}-\mathrm{O}_{\text {water }}-\mathrm{O}_{\text {Asp85 }}$ angle in $\mathrm{BR}$ is $106^{\circ}$ (Figure 13). As the consequence, if the water oxygen fully accepts the hydrogen bond of the Schiff base, the O-H group of water points toward the oxygen of Asp85 in BR, but not toward that of Asp75 in ASR (Figure 13). Such a small difference in angle possibly determines the hydrogen bonding strength of water molecules.

Figure 13. Schematic drawing of hydrogen bonds of the water molecule locating between the protonated Schiff base and its counterion. A part of all-trans retinal is depicted, $\beta$-ionon ring and ethylenic part from C6 to $\mathrm{C} 12$ are omitted. The numbers are the angle of the $\mathrm{N}-\mathrm{O}-\mathrm{O}$ atoms derived from the crystal structures of ASR and BR (PDB entries are 1XIO and $1 \mathrm{C} 3 \mathrm{~W}$, respectively). Hydrogen bonds are indicated by the dashed lines with their strength. This figure is reprinted with permission from Furutani et al. [20]. Copyright 2005 American Chemical Society.

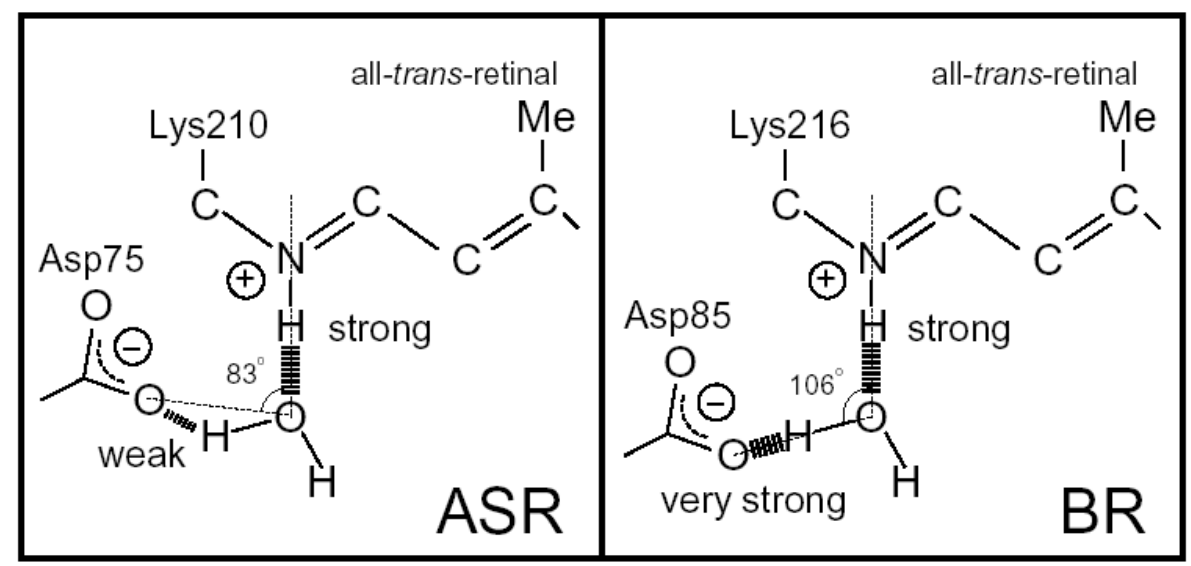


On the basis of our FTIR studies of BR mutants and other rhodopsins, we have found an interesting correlation between strongly hydrogen bonded water molecules and proton pump activity. Among various BR mutant proteins we have studied, only D85N and D212N lack strongly hydrogen bonded water molecules. Other BR mutants possess their O-D stretches at $\angle 2,400 \mathrm{~cm}^{-1}$, which include T46V, R82Q, R82Q/D212N, T89A, D96N, D115N, Y185F, and E204Q [54]. Among these mutants, only D85N and D212N do not pump protons. Therefore, strongly hydrogen bonded water molecules are only found in the proteins exhibiting proton pumping activities. The correlation between proton pumping activity and strongly hydrogen bonded water molecules is true not only for BR mutants but also for various rhodopsins. Whether rhodopsins possess strongly hydrogen bonded water molecules has been examined systematically. We found that BR and pharaonis phoborhodopsin $[26,36,46]$, both of which pump protons, possess such water molecules (O-D stretch at $<2,400 \mathrm{~cm}^{-1}$ in $\mathrm{D}_{2} \mathrm{O}$ ). In contrast, strongly hydrogen bonded water molecules were not observed for halorhodopsin [55], Neurospora rhodopsin [37], and bovine rhodopsin [56]. It is known that none of them pumps protons. Such comprehensive studies of archaeal and visual rhodopsins have thus revealed that strongly hydrogen bonded water molecules are only found in the proteins exhibiting proton pumping activities. Taken together, these results for ASR suggest that the strong hydrogen bonds of water molecules and their transient weakening may be essential for the proton pumping function of rhodopsins.

\section{FTIR Study of the Photoisomerization Processes in the 13-cis and All-trans Forms of Anabaena Sensory Rhodopsin at $77 \mathrm{~K}$}

We then extended the low-temperature spectroscopic study at $77 \mathrm{~K}$ to the 13-cis, 15-syn form of ASR (13C-ASR). HPLC analysis revealed that light-adapted ASR with $>560 \mathrm{~nm}$ light at $4{ }^{\circ} \mathrm{C}$ possesses 78\% 13C-ASR, while dark-adapted ASR has AT-ASR predominantly (97\%). Then, we established the illumination conditions to measure the difference spectra between 13C-ASR and its $\mathrm{K}$ state without subtracting the difference between AT-ASR and its K state. Spectral comparison between 13C-ASR and AT-ASR provided useful information on structure and structural changes upon retinal photoisomerization in ASR. In particular, previous X-ray crystallographic study of ASR reported the same protein structure for 13C-ASR and AT-ASR [16], whereas the present FTIR study revealed that protein structural changes upon retinal photoisomerization were significantly different between 13C-ASR and AT-ASR. The differences were seen for HOOP modes of the retinal chromophore, amide I, cysteine S-H stretch, the Schiff base N-D stretch, and water O-D stretch modes. These must trigger different global protein structural changes in each photoreaction cycle leading to the observed photochromic behavior.

Dark-adapted ASR is predominantly in the all-trans form, while the light adaptation process increases concentration of the 13-cis form [16,57]. This is in contrast to the case of BR, where light adaptation leads to a complete conversion into the all-trans form [58]. In this study, we attempted to establish the illumination conditions to accumulate the 13-cis form for DM-solubilized and PC-liposome-based ASR samples, using HPLC column chromatography. Panels a and b of Figure 14 show that the dark-adapted ASR (solid lines) possesses $95.5 \%$ and $97.1 \%$ all-trans form for the DM-solubilized and PC-liposome-based samples, respectively. On the other hand, illumination of ASR 
with $>560 \mathrm{~nm}$ light for $1 \mathrm{~min}$ at $4{ }^{\circ} \mathrm{C}$ results in accumulation of 13C-ASR. HPLC analysis showed that the light-adapted ASR possesses $78.1 \%$ and $77.9 \%$ of the 13-cis form for the DM-solubilized and PC-liposome-based samples, respectively. Thus, the isomeric composition was not influenced by the reconstitution. Dark adaptation was a slow process, with half-time $>1 \mathrm{~h}$ at $4{ }^{\circ} \mathrm{C}$ (data not shown). It should be noted that Sineshchekov et al. estimated the ratio of all-trans and 13-cis form to be 67:33 in the dark-adapted ASR and 20:80 in the light-adapted ASR [57]. The different value in the dark-adapted state may originate from the lipids for reconstitution (E.coli membrane in [57]).

Figure 14. HPLC of chromophores extracted from ASR in DM micelles (a) and in PC liposomes (b). The detection beam was set at $360 \mathrm{~nm}$. After the extraction, retinal oxime exists in 15-syn and 15-anti form. In the shown range of retention times, only the 15-syn form appears. We used area of both 15-syn and 15-anti forms for calculation of isomeric ratios. Dark-adapted ASR (solid lines) is in the all-trans form predominantly [AT-ASR; $95.5 \pm 0.8 \%$ in (a) and $97.1 \pm 0.1 \%$ in (b)], while light-adapted ASR (dotted lines) possesses more of the 13-cis form [13C-ASR; 78.1 $\pm 1.2 \%$ in (a) and $77.9 \pm 1.7 \%$ in (b)]. This figure is reprinted with permission from Kawanabe et al. [59]. Copyright 2006 American Chemical Society.

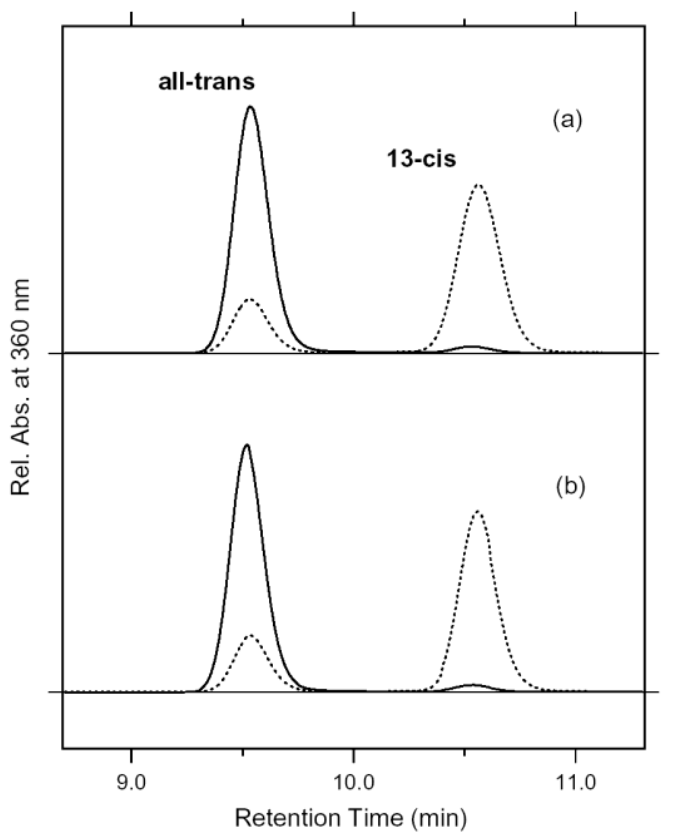

A hydrated film of ASR in PC liposomes was light-adapted as described above and then cooled to $77 \mathrm{~K}$, followed by illumination at $501 \mathrm{~nm}$. Figure 15a shows the light minus dark difference FTIR spectra of the light-adapted ASR. Vibrational bands at 1,218(-), 1,199(+), 1,196(-), and 1,189(+) $\mathrm{cm}^{-1}$ also appear in the AT-ASR $\mathrm{K}_{\mathrm{K}}$ minus AT-ASR (Figure 15c) [20], indicating that the conversion of ATASR to AT-ASR $\mathrm{K}_{\mathrm{K}}$ is included in the spectrum of Figure 15a. On the other hand, Figure 15a possesses additional strong peaks at $1,184(-)$ and $1,178(+) \mathrm{cm}^{-1}$, suggesting the involvement of the photoreaction of 13C-ASR. In the previous study for AT-ASR, we illuminated AT-ASR $\mathrm{K}_{\mathrm{K}}$ at $>590 \mathrm{~nm}$ for the photoreversion to AT-ASR [20]. In the present study, subsequent illuminations at $>560$ and $501 \mathrm{~nm}$ yielded the spectra shown in Figure 15b (dotted and solid lines, respectively). Lack of the 
bands at $1,218,1,199,1,196$, and $1,189 \mathrm{~cm}^{-1}$ strongly suggests that the spectra should not contain the photoreaction of AT-ASR. In other words, the solid line in Figure $15 \mathrm{~b}$ corresponds to the $13 \mathrm{C}-\mathrm{ASR}_{\mathrm{K}}$ minus 13C-ASR spectrum. In fact, the spectrum of Figure 15a is well constructed from the solid lines in Figure $15 \mathrm{~b}, \mathrm{c}$ (data not shown). In this way, we obtained the $13 \mathrm{C}-\mathrm{ASR}_{\mathrm{K}}$ minus $13 \mathrm{C}$-ASR difference FTIR spectra without any subtraction of spectral contribution from AT-ASR.

Figure 15. Difference FTIR spectra in the $1,240-1,130 \mathrm{~cm}^{-1}$ region measured at $77 \mathrm{~K}$ (in $\mathrm{H}_{2} \mathrm{O}$ ), where the spectra before illumination were subtracted from those after illumination. Light-adapted ASR that contains 13C-ASR (78\%) and AT-ASR (22\%) was first illuminated with $501 \mathrm{~nm}$ light for $1 \mathrm{~min}$ (a). Then, illumination at >560 nm for $1 \mathrm{~min}$ converted a part of the photoproducts in (a) to the original state (dotted line in b). Subsequent illumination with $501 \mathrm{~nm}$ light yields the difference spectrum (solid line in b), which is a mirror image of the dotted spectrum. Repeated illuminations at $>560 \mathrm{~nm}$ and at $501 \mathrm{~nm}$ yield the identical spectra. (c) The AT-ASR $\mathrm{K}_{\mathrm{K}}$ minus AT-ASR spectra are reproduced from Furutani et al. [20]. This figure is reprinted with permission from Kawanabe et al. [59]. Copyright 2006 American Chemical Society.

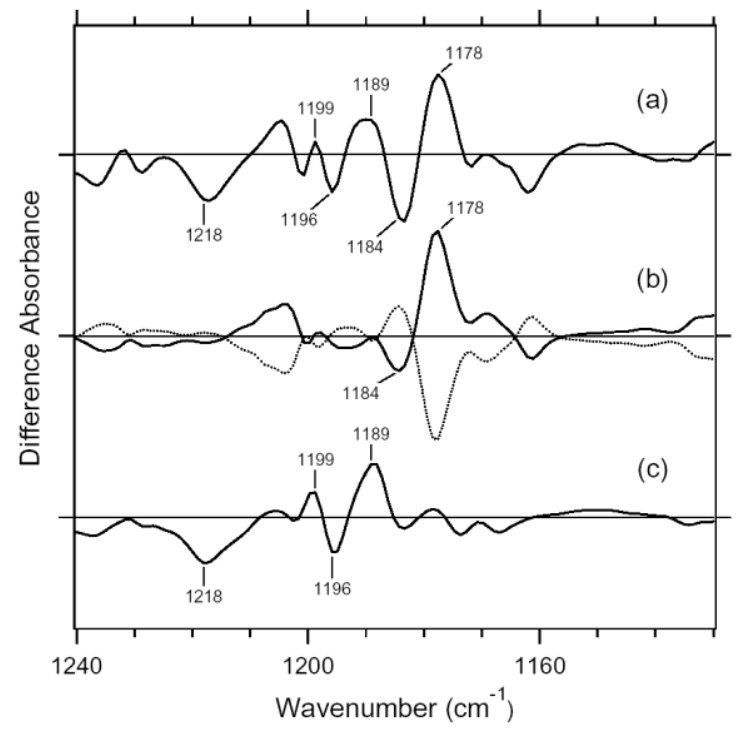

It is likely that the photoequilibrium between AT-ASR and AT-ASR $\mathrm{K}_{\mathrm{K}}$ is not changed between illuminations at $501 \mathrm{~nm}$ and at $>560 \mathrm{~nm}$, so that further illumination with $501 \mathrm{~nm}$ and >560 nm yielded the difference spectra between $13 \mathrm{C}$-ASR and $13 \mathrm{C}-\mathrm{ASR}_{\mathrm{K}}$. In this way, we obtained the $13 \mathrm{C}-\mathrm{ASR}_{\mathrm{K}}$ minus 13C-ASR difference FTIR spectra without any subtraction of spectral contribution from AT-ASR.

3.1. Comparison of the Difference Infrared Spectra of the Photoreactions of 13C-ASR and AT-ASR at $77 \mathrm{~K}$ in the $1,770-870 \mathrm{~cm}^{-1}$ Region

Figure 16 shows the $13 \mathrm{C}-\mathrm{ASR}_{\mathrm{K}}$ minus $13 \mathrm{C}$-ASR (a) and the AT-ASR $\mathrm{K}_{\mathrm{K}}$ minus AT-ASR spectra (b), which were measured at $77 \mathrm{~K}$ upon hydration with $\mathrm{H}_{2} \mathrm{O}$ (solid lines) and $\mathrm{D}_{2} \mathrm{O}$ (dotted lines). In Figure 16a, the negative band at $1,539 \mathrm{~cm}^{-1}$ corresponds to the ethylenic stretching vibration of the 13-cis chromophore in ASR, which exhibits the absorption maximum at $537 \mathrm{~nm}$ [16]. The frequency 
is in good agreement with the well-known linear correlation between the ethylenic stretching frequencies and absorption maxima for various retinal proteins [29]. Illumination results in the spectral downshift to $1,527 \mathrm{~cm}^{-1}$, indicating formation of the red-shifted $\mathrm{K}$ intermediate $\left(13 \mathrm{C}-\mathrm{ASR}_{\mathrm{K}}\right)$.

C-C stretching vibrations of the retinal in the $1,300-1,150 \mathrm{~cm}^{-1}$ region are sensitive to the local structure of the chromophore. In the $13 \mathrm{C}-\mathrm{ASR}_{\mathrm{K}}$ minus $13 \mathrm{C}$-ASR spectrum in $\mathrm{H}_{2} \mathrm{O}$, peaks are observed at $1,277(+), 1,258(-), 1,204(+), 1,184(-), 1,178(+)$, and 1,161(-) $\mathrm{cm}^{-1}$ (Figure 16a, solid line).

In the case of the 13-cis form of BR, appearance of a peak pair at $1,185(-)$ and $1,177(+) \mathrm{cm}^{-1}$ was regarded as a marker of the formation of the all-trans photoproduct [33]. Similar bands at 1,184(-) and $1,178(+) \mathrm{cm}^{-1}$ for $13 \mathrm{C}$-ASR strongly suggest that $13 \mathrm{C}-\mathrm{ASR}_{\mathrm{K}}$ possesses the all-trans chromophore produced by photoisomerization of the $\mathrm{C} 13=\mathrm{C} 14$ bond. As in the case of $\mathrm{BR}$, the $1,184(-) / 1,178(+) \mathrm{cm}^{-1}$ bands are insensitive to the H-D exchange (Figure 16a, dotted line), being thus assignable to $\mathrm{C} 10-\mathrm{C} 11$ stretching vibration [33]. Strong positive peaks at 1,277 and $1,204 \mathrm{~cm}^{-1}$ in $\mathrm{H}_{2} \mathrm{O}$ and at $1,231 \mathrm{~cm}^{-1}$ in $\mathrm{D}_{2} \mathrm{O}$ were also observed for the 13-cis form of BR, where positive peaks at $1,205 \mathrm{~cm}^{-1}$ in $\mathrm{H}_{2} \mathrm{O}$ and at $1,234 \mathrm{~cm}^{-1}$ in $\mathrm{D}_{2} \mathrm{O}$ were assigned to be $\mathrm{C} 14-\mathrm{C} 15$ stretching vibrations [33]. Therefore, corresponding peaks at $1,204 \mathrm{~cm}^{-1}$ in $\mathrm{H}_{2} \mathrm{O}$ and at $1,231 \mathrm{~cm}^{-1}$ in $\mathrm{D}_{2} \mathrm{O}$ are assignable to the C14-C15 stretching vibration of $13 \mathrm{C}-\mathrm{ASR}_{\mathrm{K}}$. Spectral coincidence between BR and ASR implies similar chromophore structures of their 13-cis forms and respective K states. Hydrogen-out-of-plane (HOOP), $\mathrm{N}-\mathrm{D}$ in-plane bending, and methyl rocking vibrations are observed in the $1,110-890 \mathrm{~cm}^{-1}$ region, and the presence of strong HOOP modes represents distortions of the retinal molecule [60]. The AT-ASR $\mathrm{K}_{\mathrm{K}}$ minus AT-ASR spectra exhibit two strong peaks at 968 and $957 \mathrm{~cm}^{-1}$ (Figure 16b).

Figure 16. The $13 \mathrm{C}-\mathrm{ASR}_{\mathrm{K}}$ minus $13 \mathrm{C}-\mathrm{ASR}$ (a) and the $\mathrm{AT}-\mathrm{ASR}_{\mathrm{K}}$ minus AT-ASR (b) spectra ( $\mathrm{pH} 7$ ) in the $1,800-800 \mathrm{~cm}^{-1}$ region measured at $77 \mathrm{~K}$ upon hydration with $\mathrm{H}_{2} \mathrm{O}$ (solid line) and $\mathrm{D}_{2} \mathrm{O}$ (dotted line), respectively. The spectra in panel $\mathrm{b}$ are reproduced from Furutani et al. [20]. One division of the y-axis corresponds to 0.007 absorbance units. This figure is reprinted with permission from Kawanabe et al. [59]. Copyright 2006 American Chemical Society.

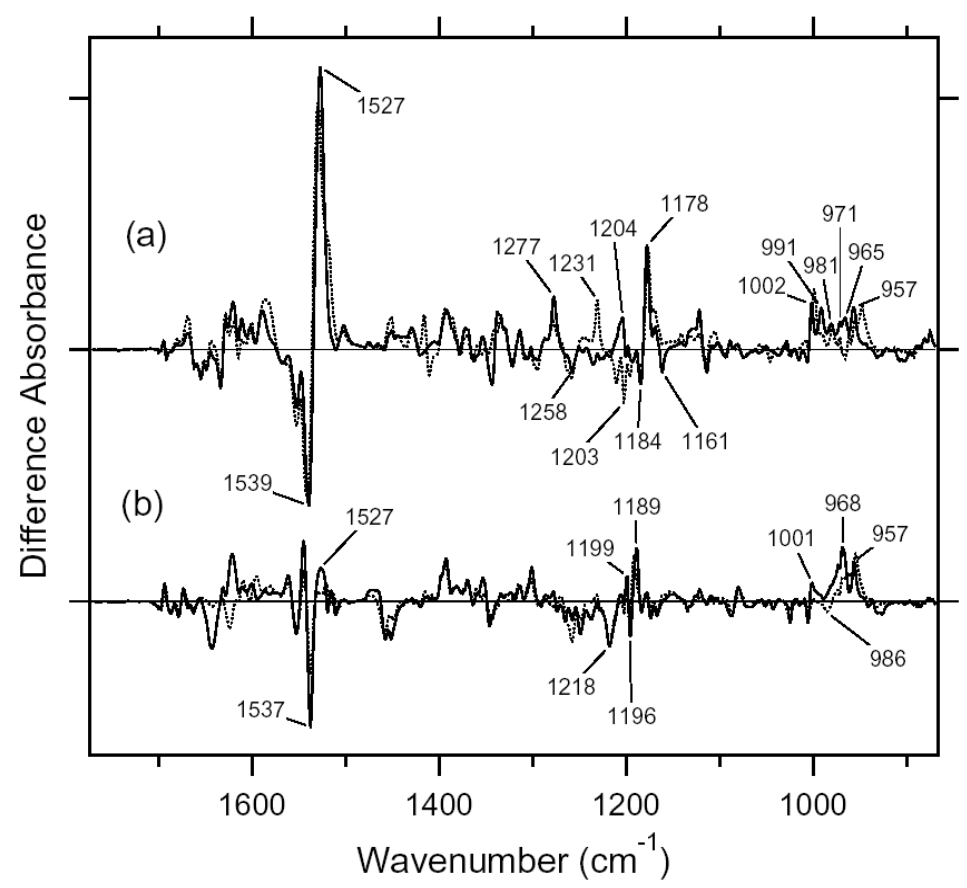


In contrast, many positive bands were observed in the $13 \mathrm{C}-\mathrm{ASR}_{\mathrm{K}}$ minus $13 \mathrm{C}$-ASR spectra, whose frequencies are at 1,002, 991, 981, 971, 965, and $957 \mathrm{~cm}^{-1}$ (Figure 16a). This observation suggests that the chromophore of $13 \mathrm{C}-\mathrm{ASR}_{\mathrm{K}}$ is more distorted along the polyene chain than that of AT-ASR .

Figure 17 shows the $13 \mathrm{C}-\mathrm{ASR}_{\mathrm{K}}$ minus $13 \mathrm{C}$-ASR (a) and the AT-ASR $\mathrm{K}_{\mathrm{K}}$ minus AT-ASR spectra (b) in the $1,750-1,550 \mathrm{~cm}^{-1}$ region. Amide I vibrations appear in this frequency region together with the $\mathrm{C}=\mathrm{N}$ stretching vibration of the protonated retinal Schiff base. In general, the former is insensitive to the H-D exchange, whereas the latter exhibits downshift in $\mathrm{D}_{2} \mathrm{O}$. In the case of AT-ASR, a prominent peak pair at $1,642(-)$ and $1,621(+) \mathrm{cm}^{-1}$ is assignable to the $\mathrm{C}=\mathrm{N}$ stretchings of AT-ASR and AT-ASR $\mathrm{K}$, respectively, because of the spectral shifts to $1,624(-)$ and $1,610(+) \mathrm{cm}^{-1}$ in $\mathrm{D}_{2} \mathrm{O}$ (Figure 17b). In fact, we observed the downshift of the bands at 1,642(-) and $1,621(+) \mathrm{cm}^{-1}$ by $10 \mathrm{~cm}^{-1}$ for $\left[\zeta_{-}{ }^{15} \mathrm{~N}\right]$ lysine-labeled ASR, indicating that they originate from the $\mathrm{C}=\mathrm{N}$ stretching vibrations (data not shown). It should be noted that the spectral changes of amide I vibrations at $1,660-1,630 \mathrm{~cm}^{-1}$ are small in AT-ASR $\mathrm{K}_{\mathrm{K}}$ minus AT-ASR, which is clearly seen in $\mathrm{D}_{2} \mathrm{O}$ (Figure $17 \mathrm{~b}$, dotted line), suggesting that no structural changes of the peptide backbone occur in AT-ASR upon retinal isomerization. The spectral features are quite different for 13C-ASR. Figure 17a shows the presence of the H-D exchange independent bands in the 1,660-1,630 $\mathrm{cm}^{-1}$ region, located at 1,669(+), 1,662(-), 1,655(-), 1,649(-), $1,644(+), 1,634(-)$ and $1,628(+) \mathrm{cm}^{-1}$. This suggests perturbation of the peptide backbone upon retinal photoisomerization of $13 \mathrm{C}$-ASR. In particular, the peaks at 1,662, 1,655, and 1,649 $\mathrm{cm}^{-1}$ are ascribable to the amide I vibrations of the $\alpha$-helix. Helical perturbation may be correlated with many peaks of the HOOP vibrations in $13 \mathrm{C}-\mathrm{ASR}_{\mathrm{K}}$.

Figure 17. The $13 \mathrm{C}-\mathrm{ASR}_{\mathrm{K}}$ minus $13 \mathrm{C}-\mathrm{ASR}$ (a) and the $\mathrm{AT}-\mathrm{ASR}_{\mathrm{K}}$ minus AT-ASR (b) spectra ( $\mathrm{pH} 7$ ) in the $1,750-1,550 \mathrm{~cm}^{-1}$ region, mostly representing vibrations of the protein moiety. The sample was hydrated with $\mathrm{H}_{2} \mathrm{O}$ (solid lines) or $\mathrm{D}_{2} \mathrm{O}$ (dotted lines). One division of the y-axis corresponds to 0.0025 absorbance units. This figure is reprinted with permission from Kawanabe et al. [59]. Copyright 2006 American Chemical Society.

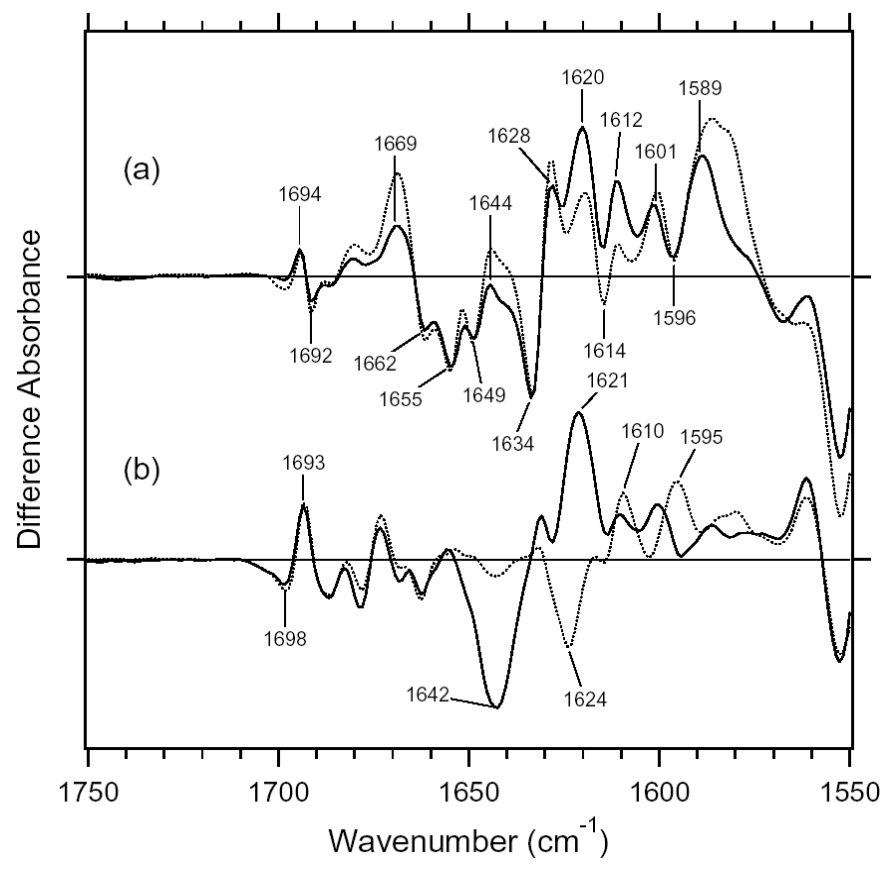


Unlike in AT-ASR (Figure 17b), the $13 \mathrm{C}-\mathrm{ASR}_{\mathrm{K}}$ minus 13C-ASR spectra (Figure 17a) do not show $\mathrm{H}-\mathrm{D}$ exchange dependent bands clearly. This indicates that the $\mathrm{C}=\mathrm{N}$ stretching vibrations are not clearly observed in the spectra. Reproducible differences between $\mathrm{H}_{2} \mathrm{O}$ and $\mathrm{D}_{2} \mathrm{O}$ samples in Figure 17a suggest that the $\mathrm{C}=\mathrm{N}$ stretching vibrations are present in this frequency region. In fact, bands at $1,640-1,620 \mathrm{~cm}^{-1}$ were sensitive to $\left[\zeta{ }^{15} \mathrm{~N}\right]$ lysine labeling (not shown). However, the absence of clear peaks of the $\mathrm{C}=\mathrm{N}$ stretch requests spectral analysis using double difference spectra. The $\mathrm{C}=\mathrm{N}$ stretching vibrations have been regarded as an important marker, because the difference in frequency between $\mathrm{H}_{2} \mathrm{O}$ and $\mathrm{D}_{2} \mathrm{O}$ samples probes hydrogen-bonding strength of the Schiff base [39,40]. In the present study, however, we discuss the hydrogen-bonding strength of the Schiff base by use of the N-D stretching in $\mathrm{D}_{2} \mathrm{O}$ (see below), which is the more direct probe [41].

In the carboxylic $\mathrm{C}=\mathrm{O}$ stretching frequency region $\left(>1,700 \mathrm{~cm}^{-1}\right)$, there are no bands for $13 \mathrm{C}$ - and

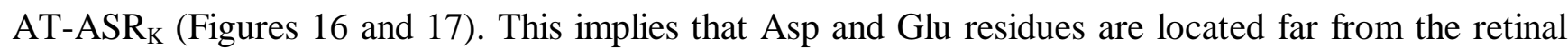
even if they are protonated. In the $\mathrm{BR}_{\mathrm{K}}$ minus $\mathrm{BR}$ difference spectra, the bands at 1,742(-) and 1,733(+) $\mathrm{cm}^{-1}$ were assigned to the $\mathrm{C}=\mathrm{O}$ stretching vibrations of the protonated Asp115 [42]. ASR has a glutamine residue (Gln109) at the corresponding position, whose vibrational bands are probably observed at 1,698(-) and 1,693(+) $\mathrm{cm}^{-1}$ for AT-ASR (Figure 17b). Similar bands were also observed at 1,704(-) and 1,700(+) $\mathrm{cm}^{-1}$ in the difference spectra of $p \mathrm{pR}$, which has an asparagine residue at the corresponding position [43]. Therefore, it can be suggested that the structural changes occurring around Asp115 in BR are common for ASR, BR, and $p$ pR. Figure 17a shows the bands at 1,694(+) and $1,692(-) \mathrm{cm}^{-1}$ for $13 \mathrm{C}$-ASR, which can be assigned to the $\mathrm{C}=\mathrm{O}$ stretch of $\mathrm{Gln} 109$. It is likely that the $\mathrm{C}=\mathrm{O}$ stretching vibrations of Asp115 in BR are dependent on the isomeric form as well.

Figure 18. The $13 \mathrm{C}-\mathrm{ASR}_{\mathrm{K}}$ minus $13 \mathrm{C}-\mathrm{ASR}$ (a) and the $\mathrm{AT}-\mathrm{ASR}_{\mathrm{K}}$ minus AT-ASR (b) spectra ( $\mathrm{pH} 7$ ) in the 2,580-2,500 $\mathrm{cm}^{-1}$ region, which correspond to $\mathrm{S}-\mathrm{H}$ stretching vibrations of cysteine residues. The sample was hydrated with $\mathrm{H}_{2} \mathrm{O}$. One division of the $\mathrm{y}$-axis corresponds to 0.0003 absorbance units. This figure is reprinted with permission from Kawanabe et al. [59]. Copyright 2006 American Chemical Society.

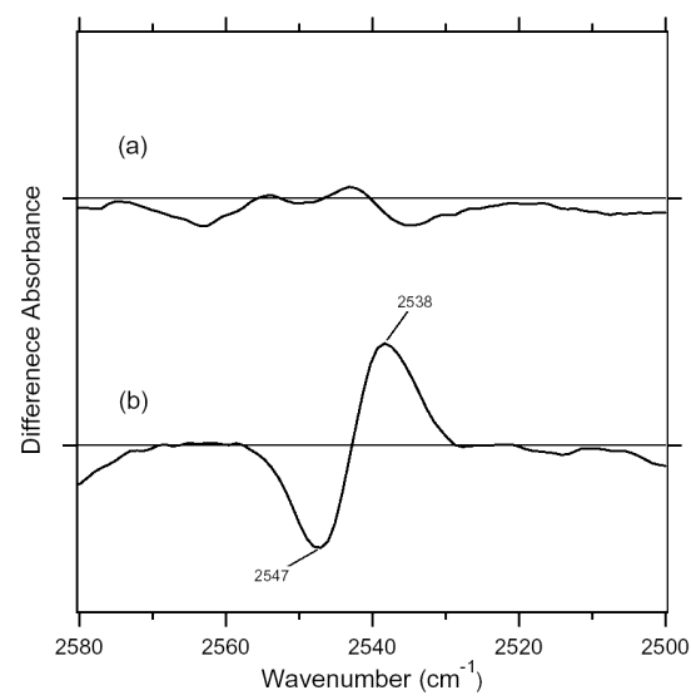




\subsection{S-H Stretching Vibrations of Cysteine Residues}

Figure 18 shows the $13 \mathrm{C}-\mathrm{ASR}_{\mathrm{K}}$ minus $13 \mathrm{C}$-ASR (upper panel) and $\mathrm{AT}-\mathrm{ASR}_{\mathrm{K}}$ minus AT-ASR (lower panel) spectra in the $2,580-2,500 \mathrm{~cm}^{-1}$ region, which corresponds to $\mathrm{S}-\mathrm{H}$ stretching vibration of cysteine. As we already reported, there is a negative band at $2,547 \mathrm{~cm}^{-1}$ and a positive band at $2,538 \mathrm{~cm}^{-1}$ for AT-ASR (Figure 18b). In contrast, no spectral changes were observed for 13C-ASR, indicating that the 13-cis to all-trans isomerization in ASR does not alter the local structure of cysteines (Figure 18a). We suggested that the observed vibrational bands may be assignable to the $\mathrm{S}-\mathrm{H}$ stretching of Cys203 in previous section [20].

\subsection{Assignment of the N-D Stretching Vibrations in 13C-ASR and AT-ASR}

X-D stretching vibrations of protein and water molecules appear in the 2,750-2,000 $\mathrm{cm}^{-1}$ region for the films hydrated with $\mathrm{D}_{2} \mathrm{O}$ (Figure 19). The solid line of Figure $19 \mathrm{c}$ shows the AT-ASR $\mathrm{K}_{\mathrm{K}}$ minus AT-ASR spectrum reported earlier [20]. On the other hand, the 13C-ASR ${ }_{K}$ minus $13 \mathrm{C}-\mathrm{ASR}$ spectrum (solid line of Figure 19a) is also obtained previously [59].

Figure 19. The $13 \mathrm{C}-\mathrm{ASR}_{\mathrm{K}}$ minus $13 \mathrm{C}-\mathrm{ASR}$ (a) and the $\mathrm{AT}-\mathrm{ASR}_{\mathrm{K}}$ minus AT-ASR (c) spectra (pH 7) in the 2,750-2,000 $\mathrm{cm}^{-1}$ region for $\left[\zeta{ }^{15} \mathrm{~N}\right]$ lysine-labeled (dotted line) and unlabeled (solid line) ASR. Double difference spectra in (a) and (c) (solid line minus dotted line) are shown in (b) and (d), respectively. The samples were hydrated with $\mathrm{D}_{2} \mathrm{O}$, and spectra were measured at $77 \mathrm{~K}$. One division of the y-axis corresponds to 0.0007 absorbance units. These figures are reprinted with permission from Kawanabe et al. [59]. Copyright 2006 American Chemical Society.

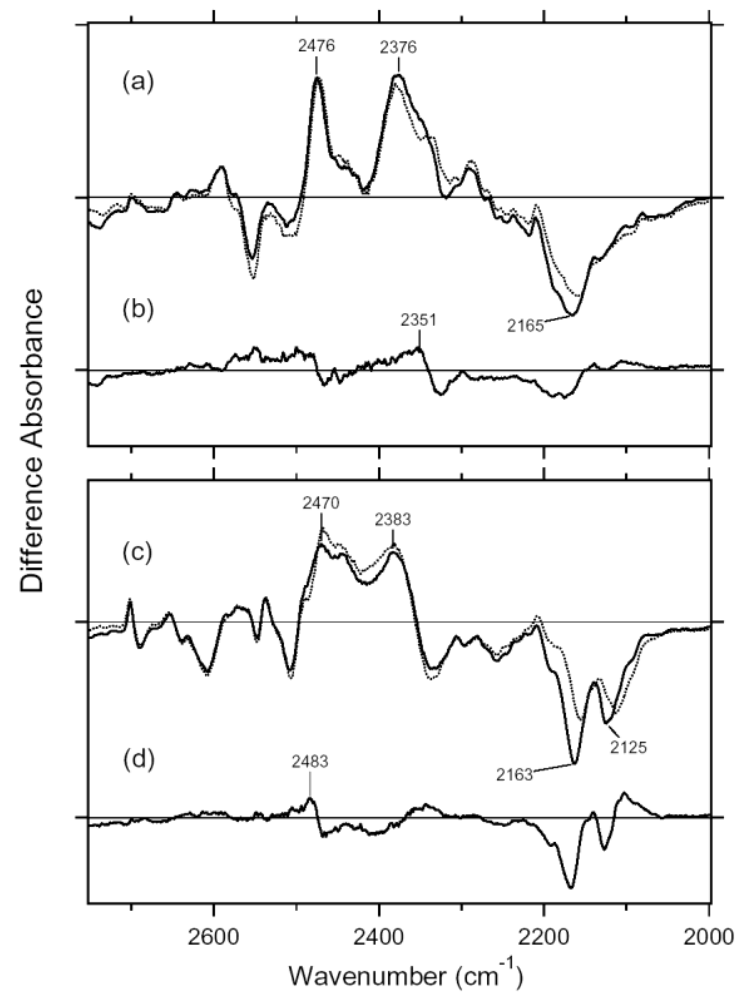


Since the N-D stretching vibrations of the Schiff base should be present in this frequency region, we then attempted to assign them by use of the $\left[\zeta_{-}{ }^{15} \mathrm{~N}\right]$ lysine-labeled ASR sample. Figure 19a compares the $13 \mathrm{C}-\mathrm{ASR}_{\mathrm{K}}$ minus $13 \mathrm{C}$-ASR spectra between $\left[\zeta_{-}{ }^{15} \mathrm{~N}\right]$ lysine-labeled (dotted line) and unlabeled (solid line) ASR. Clear isotope-induced spectral downshift was observed for intense positive and negative bands at 2,376 and $2,165 \mathrm{~cm}^{-1}$, respectively. Other bands are identical between $\left[\zeta_{-}{ }^{15} \mathrm{~N}\right]$ lysinelabeled and unlabeled 13C-ASR. Thus, we are able to conclude that the N-D stretching vibrations of the Schiff base are present in this frequency region. It should however be noted that the strong positive peak at $2,376 \mathrm{~cm}^{-1}$ probably contains other vibrations because the isotope effect was observed in the broad range of 2,370-2,320 $\mathrm{cm}^{-1}$ (Figure 19a).

In fact, the AT-ASR $\mathrm{K}_{\mathrm{K}}$ minus AT-ASR spectra contain such peak at 2,383 $\mathrm{cm}^{-1}$ as well (Figure 19c), which may originate from amide A vibrations. By use of double difference spectra from the data shown in Figure 19a, we determined that the N-D stretching vibration of the Schiff base in $13 \mathrm{C}-\mathrm{ASR}_{\mathrm{K}}$ is located at $2,351 \mathrm{~cm}^{-1}$ (Figure 19b).

Figure $19 \mathrm{c}$ compares the AT-ASR $\mathrm{K}_{\mathrm{K}}$ minus AT-ASR spectra between $\left[\zeta_{-}{ }^{15} \mathrm{~N}\right]$ lysine-labeled (dotted line) and unlabeled (solid line) ASR. Clear isotope-induced spectral downshift was observed for the two negative bands at 2,163 and $2,125 \mathrm{~cm}^{-1}$, indicating that the bands originate from $\mathrm{N}-\mathrm{D}$ stretching vibrations of the Schiff base in AT-ASR. Additionally, the positive spectral feature at $2,470 \mathrm{~cm}^{-1}$ exhibits isotope shift from $\left[\zeta^{15} \mathrm{~N}\right]$ lysine labeling as well. By use of double difference spectra from the data shown in Figure 19c, we determined that the N-D stretching vibration of the Schiff base in AT$\mathrm{ASR}_{\mathrm{K}}$ is located at $2,483 \mathrm{~cm}^{-1}$ (Figure 19d). The positive peak at 2,470 $\mathrm{cm}^{-1}$ probably contains other vibrations such as the O-D stretching vibrations of Thr79. In BR, the O-D frequencies of Thr89, the homologue of Thr79 in ASR, are 2,507 and 2,466 $\mathrm{cm}^{-1}$ for $\mathrm{BR}$ and $\mathrm{BR}_{\mathrm{K}}$, respectively [49]. A similar positive band was also observed at $2,476 \mathrm{~cm}^{-1}$ for 13C-ASR (Figure 19a).

Thus, by use of $\left[\zeta{ }^{15} \mathrm{~N}\right]$ lysine-labeled ASR, we identified the N-D stretching vibrations of the Schiff base at 2,163 and $2,125 \mathrm{~cm}^{-1}$ for AT-ASR and at $2,165 \mathrm{~cm}^{-1}$ for $13 \mathrm{C}$-ASR. This indicates that the hydrogen-bonding strength is very similar for the two isomeric forms, being slightly stronger in AT-ASR. The X-ray crystallographic structure reported the presence of a water molecule in contact with the Schiff base, making it a good candidate for the hydrogen-bonding acceptor [60]. Similarity of the hydrogen bonding in AT-ASR and 13C-ASR is consistent with the X-ray structure.

We also identified the N-D stretching vibration of the Schiff base at $2,483 \mathrm{~cm}^{-1}$ for AT-ASR $\mathrm{K}_{\mathrm{K}}$ and at $2,351 \mathrm{~cm}^{-1}$ for $13 \mathrm{C}-\mathrm{ASR}_{\mathrm{K}}$. Upshifted N-D frequencies indicate that retinal isomerization weakens the hydrogen bond of the Schiff base for both AT-ASR and 13C-ASR. Nevertheless, unlike in the unphotolyzed states, the difference in frequencies for the $\mathrm{K}$ states implies the different isomerization outcomes for AT-ASR and 13C-ASR. In case of AT-ASR, the upshift of the frequency is $>300 \mathrm{~cm}^{-1}$, indicating that the hydrogen bond is significantly weakened (or broken) in AT-ASR $\mathrm{K}_{\mathrm{K}}$, presumably because of the rotational motion of the Schiff base. In contrast, the upshift of the frequency is about $200 \mathrm{~cm}^{-1}$ for $13 \mathrm{C}$-ASR. This fact suggests that the rotational motion of the Schiff base that accompanies retinal isomerization is smaller in 13C-ASR than in AT-ASR. 


\subsection{O-D Stretching Vibrations of Water in 13C-ASR and AT-ASR}

A spectral comparison between the samples hydrated with $\mathrm{D}_{2} \mathrm{O}$ and $\mathrm{D}_{2}{ }^{18} \mathrm{O}$ identifies $\mathrm{O}-\mathrm{D}$ stretching vibrations of water molecules which change their frequencies upon retinal photoisomerization. We showed the absence of the water O-D stretch at $<2,500 \mathrm{~cm}^{-1}$ for AT-ASR in previous section [20]. This observation was entirely different from the case of $\mathrm{BR}$, being consistent with the correlation between strongly hydrogen-bonded water molecules and proton pumping activity [27].

In this study, we also looked for the water bands in the $13 \mathrm{C}-\mathrm{ASR}_{\mathrm{K}}$ minus $13 \mathrm{C}-\mathrm{ASR}$ spectrum, but no water bands were found at $<2,500 \mathrm{~cm}^{-1}$ similar to AT-ASR (data not shown). This fact indicates that the bridged water molecule between the protonated Schiff base and Asp75 forms a weak hydrogen bond for both the all-trans and 13-cis form. Figure 20 shows difference FTIR spectra in the 2,750-2,520 $\mathrm{cm}^{-1}$ region, where weakly hydrogen-bonded water molecules are observed. Green-tagged bands in Figure 20 are assignable to the $\mathrm{O}-\mathrm{D}$ stretching vibrations of water because of the isotope shift. Figure $20 \mathrm{~b}$ shows that three negative peaks at 2,690,2,640, and $2,608 \mathrm{~cm}^{-1}$ were assignable to the O-D stretching vibrations of water in AT-ASR, while the bands at 2,701,2,653, and 2,573 $\mathrm{cm}^{-1}$ were assigned as water

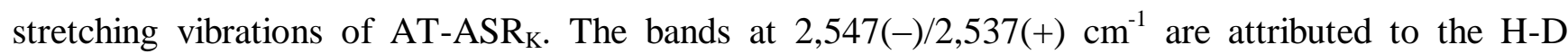
unexchangeable S-H stretching vibration of a cysteine residue as shown in Figure 18b. Figure 20a shows that the bands at 2,660(-) and 2,645 (+) $\mathrm{cm}^{-1}$ exhibit isotope shift of water. In addition, clear isotope shift was seen for the positive band at $2,589 \mathrm{~cm}^{-1}$. The negative band at $2,553 \mathrm{~cm}^{-1}$ also contains water O-D stretch, though the small downshift suggests the presence of vibrations other than that of water. Therefore, two positive and two negative peaks can be assigned as O-D stretches of water in $13 \mathrm{C}-\mathrm{ASR}$.

Figure 20. The $13 \mathrm{C}-\mathrm{ASR}_{\mathrm{K}}$ minus $13 \mathrm{C}-\mathrm{ASR}$ (a) and the $\mathrm{AT}-\mathrm{ASR}_{\mathrm{K}}$ minus AT-ASR (b) spectra ( $\mathrm{pH} 7$ ) in the $2,750-2,520 \mathrm{~cm}^{-1}$ region measured at $77 \mathrm{~K}$. Sample was hydrated with $\mathrm{D}_{2} \mathrm{O}$ (red line) or $\mathrm{D}_{2}{ }^{18} \mathrm{O}$ (blue line). Green-labeled frequencies correspond to those identified as water stretching vibrations. One division of the y-axis corresponds to 0.0004 absorbance units. This figure is reprinted with permission from Kawanabe et al. [59]. Copyright 2006 American Chemical Society.

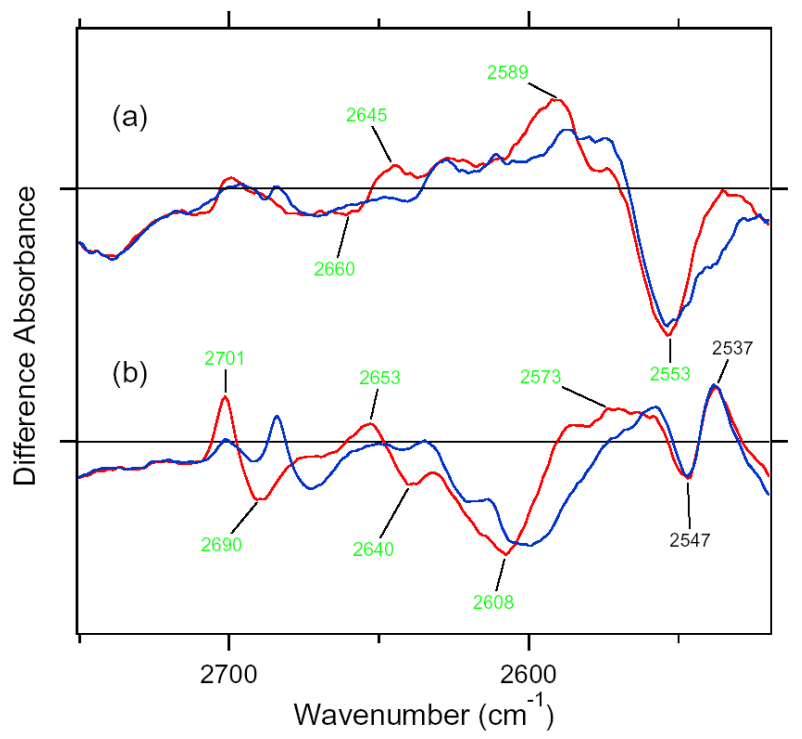




\subsection{Unique Structure of the 13-cis Form of ASR}

In this section, we compared the $13 \mathrm{C}-\mathrm{ASR}_{\mathrm{K}}$ minus $13 \mathrm{C}$-ASR and AT-ASR $\mathrm{K}_{\mathrm{K}}$ minus AT-ASR spectra obtained by means of low-temperature FTIR spectroscopy. The HPLC analysis revealed that the dark-adapted ASR is predominantly in the AT-ASR form (97\%). Then, the optimal conditions of light adaptation to accumulate 13C-ASR were established, resulting in accumulation of $78 \%$ of the 13-cis form. This unique property of ASR raises several questions on how ASR relays the signal to its ASRT and the nature of the signaling state of ASR. If there is a structural difference between 13C-ASR and AT-ASR in the ground state, it might result in different binding affinity of the ASRT for 13C-ASR and AT-ASR. But we cannot exclude a general mechanism in which the M state would be the signaling state as in other sensory rhodopsins. Although the light-adapted ASR contains AT-ASR, the appropriate illumination regime allowed us to obtain the $13 \mathrm{C}-\mathrm{ASR}_{\mathrm{K}}$ minus $13 \mathrm{C}$-ASR spectra without any subtraction of the contribution of the all-trans form (Figure 15). The spectral comparison of 13C-ASR and AT-ASR upon the retinal isomerization at $77 \mathrm{~K}$ led to detection of the structural changes specific for each isomer. In addition, we revealed the hydrogen-bonding strengths of the Schiff base in each state using $\left[\zeta_{-}{ }^{15} \mathrm{~N}\right]$ lysine-labeled ASR.

\subsection{Unphotolyzed State of 13C-ASR}

We identified the N-D stretching vibration of the Schiff base at $2,165 \mathrm{~cm}^{-1}$ for $13 \mathrm{C}$-ASR (Figure 19a). We also identified the N-D stretching vibration of the Schiff base at 2,163 and 2,125 $\mathrm{cm}^{-1}$ for AT-ASR. The similar frequencies in 13C-ASR and AT-ASR indicate that the hydrogen-bonding strength of the Schiff base is nearly identical, being slightly stronger in AT-ASR. In the case of BR, the $\mathrm{N}$-D stretching vibrations of the Schiff base were determined to be at 2,171 and 2,124 $\mathrm{cm}^{-1}$ [41]. X-ray crystallographic structures of ASR and BR reported the presence of a water molecule in contact with the Schiff base [7,16]. Therefore, similar hydrogen-bonding strength for 13C-ASR, AT-ASR, and BR suggests that the water molecule is a good hydrogen-bonding acceptor for the protonated Schiff base.

Interestingly, two peaks were observed for the N-D stretch of the Schiff base of AT-ASR (Figure 19c) and BR [20], while only one peak was observed for that of 13C-ASR (Figure 19a). Origins of the two peaks in BR, $p \mathrm{pR}$, and AT-ASR have not been well understood. Multiple vibrational modes or structural heterogeneity is a possible source of the two N-D stretches. A single peak of the 13-cis form in ASR may be useful for understanding of the nature of this mode.

We previously found that water vibrations are entirely different between AT-ASR and BR, though both possess a water molecule between the Schiff base and its counterion (Asp75 for ASR or Asp85 for BR) [7,16]. Namely, the $\mathrm{N}-\mathrm{O}_{\text {water }}-\mathrm{O}_{\text {counterion }}$ (the Schiff base nitrogen, water oxygen, and oxygen of the counterion) angle is $83^{\circ}$ and $106^{\circ}$ in ASR and BR, respectively. As the consequence, if the water oxygen fully accepts a hydrogen bond of the Schiff base, the O-H group of water points toward the oxygen of Asp85 in BR, but not toward that of Asp75 in ASR. Such a small difference in the angle can possibly determine the hydrogen-bonding strength of water molecules. We did not observe strongly hydrogen-bonded water molecules for 13C-ASR in this study (Figure 20a). This is consistent with the above argument, because the X-ray crystal structure of ASR provides a similar position of the Schiff base, the water, and Asp75 for both isomers at $2.0 \AA$ resolution [16]. 
We observed water O-D stretches of 13C-ASR at 2,660 and 2,553 $\mathrm{cm}^{-1}$ (Figure 20a), which correspond to $\mathrm{O}-\mathrm{H}$ stretches at 3,592 and $3,481 \mathrm{~cm}^{-1}$, respectively, from the spectral analysis of the $\mathrm{O}-\mathrm{H}$ stretching vibrations in $\mathrm{H}_{2} \mathrm{O}$ (not shown). The O-H stretches of AT-ASR corresponding to the O-D stretches at 2,690, 2,640, and 2,608 $\mathrm{cm}^{-1}$ in Figure $20 \mathrm{~b}$ are found at 3,636, 3,558, and 3,530 $\mathrm{cm}^{-1}$, respectively. Since only the water bridging the Schiff base and Asp75 is located close to the chromophore, it is a reasonable postulation that two water bands of ASR originate from O-D (O-H) stretches of this water molecule. In general, a water molecule has two O-H groups, and their frequencies are distributed in the wide $3,700-2,700 \mathrm{~cm}^{-1}$ region depending on their coupling and hydrogen-bonding strength [61]. Gaseous water exhibits asymmetric and symmetric stretching modes at 3,755 and $3,657 \mathrm{~cm}^{-1}$, respectively, and the stretching frequency is lowered as its hydrogen bonding becomes stronger [22]. It must be noted that the hydrogen bonding strengths of the two O-H groups are probably not equivalent in the restricted protein environment, which breaks the $\mathrm{C}_{2 \mathrm{v}}$-type symmetry. In such $\mathrm{C}_{\mathrm{S}}$-type symmetry, one $\mathrm{O}-\mathrm{H}$ is hydrogen bonded and the other $\mathrm{O}-\mathrm{H}$ is unbonded, and their frequencies are widely split. That is the case for the bridged water of BR, where such decoupling of the two stretching modes occurs [54]. Consequently, one O-D stretch of water is at $2,171 \mathrm{~cm}^{-1}$, while another O-D stretch of water is at $2,636 \mathrm{~cm}^{-1}$. We suggested that the former points toward Asp85, while the latter points toward Asp212 [54]. Nonsymmetrical bonding of the water molecule in BR is presumably important for the function $[23,26]$.

Figure 21. (Left) The X-ray structure around retinal Schiff base. Yellow retinal is all-trans form and blue retinal is 13-cis form. (Right) The diagram of the $\mathrm{ASR}_{\mathrm{K}}$ minus ASR infrared spectra in X-D vibration region. It shows only N-D stretch of the Schiff base. This figure is reprinted with permission from TOC of Kawanabe et al [59]. Copyright 2006 American Chemical Society.
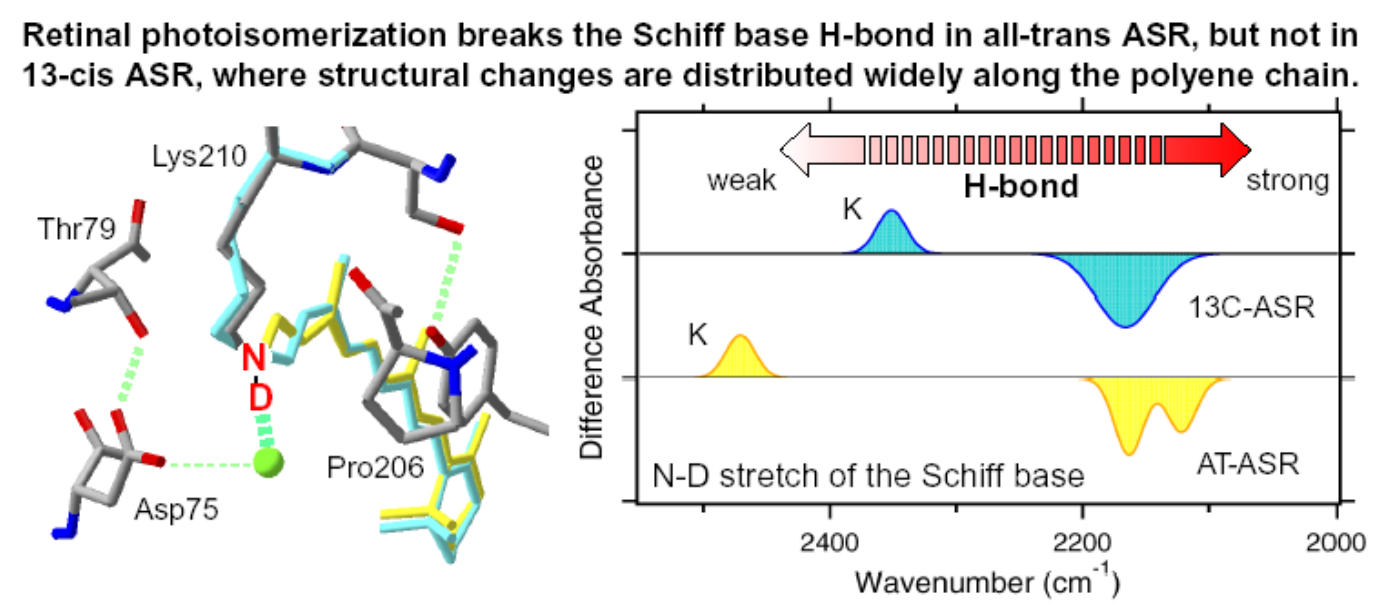

In the case of 13C-ASR, the frequency difference between the O-D stretches is about $100 \mathrm{~cm}^{-1}$. Corresponding $\mathrm{O}-\mathrm{H}$ stretches are also about $100 \mathrm{~cm}^{-1}$ different, being comparable to the gaseous water. Therefore, stretching vibrations of the water molecule are presumably coupled in 13C-ASR, where anti-symmetric and symmetric O-D stretches are located at 2,660 and 2,553 $\mathrm{cm}^{-1}$, respectively. The situation is probably similar for AT-ASR, where two out of the three bands at 2,690, 2,640, 
and 2,608 $\mathrm{cm}^{-1}$ originate from the O-D stretches of the bridging water. The presence of the additional

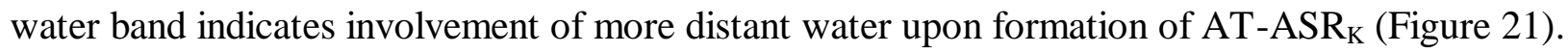

\subsection{Photoisomerization Process of 13C-ASR in Comparison with that of AT-ASR}

Upon light absorption in 13C-ASR, photoisomerization probably takes place at the $\mathrm{C} 13=\mathrm{C} 14$ (double) bond, leading from the 13-cis, 15-syn to the all-trans, 15-syn form. It is generally accepted that the primary $\mathrm{K}$ intermediate is a high-energy state for retinal proteins. Chromophore distortion is one of the characteristic features of such high energy state, and HOOP vibrations monitor the chromophore distortion. The appearance of numerous HOOP modes in $13 \mathrm{C}-\mathrm{ASR}_{\mathrm{K}}$ vs. just two in AT-ASR (Figure 16) implies that the chromophore distortion in $13 \mathrm{C}-\mathrm{ASR}_{\mathrm{K}}$ is distributed more widely along the polyene chain. In other words, chromophore distortion is more localized in the Schiff base region for AT-ASR $\mathrm{K}_{\mathrm{K}}$. Such difference in HOOP modes is presumably correlated with the other observations including amide I, cysteine S-H stretch, the Schiff base N-D stretch, and water O-D stretch modes, as discussed below.

Amide I vibrations of the $\alpha$-helix were clearly observed for the transition from 13C-ASR to $13 \mathrm{C}-\mathrm{ASR}_{\mathrm{K}}$ as shown by the negative bands at $1,662,1,655$, and $1,649 \mathrm{~cm}^{-1}$ in Figure $17 \mathrm{a}$. This is reasonable because the retinal chromophore is surrounded by $\alpha$-helices. In addition, the bands at $1,634(-) / 1,628(+) \mathrm{cm}^{-1}$ are also ascribable to amide I vibration. In contrast, fewer structural changes reported by amide I vibrations were observed for the transition from AT-ASR to AT-ASR $\mathrm{K}_{\mathrm{K}}$ as we showed previously [20]. Instead, it was suggested that imide I vibration, possibly due to Pro206, was greatly altered [20]. Several amide I changes observed only for 13C-ASR are consistent with the picture obtained from the HOOP analysis, suggesting that extensive structural changes take place in $13 \mathrm{C}-\mathrm{ASR}_{\mathrm{K}}$.

No structural perturbation was observed for S-H groups of cysteines in 13C-ASR, whereas there is a negative band at $2,547 \mathrm{~cm}^{-1}$ and a positive band at $2,538 \mathrm{~cm}^{-1}$ for AT-ASR (Figure 18). This indicates that only the all-trans to 13-cis isomerization leads to the alteration of the local structure of a cysteine in ASR. We previously suggested that among the three cysteines of ASR, Cys203 in helix G is the most likely candidate for this band. Cys203 is near Pro206 and close to the Schiff base region. Replacement of Cys 203 by Ala results in a red-shifted $\lambda_{\max }(553 \mathrm{~nm})$ relative to the wildtype ASR (unpublished data). This suggests that the Schiff base region is more perturbed in AT-ASR $R_{K}$ than in $13 C-A_{S R}$. The N-D stretching frequency of the Schiff base in $13 \mathrm{C}-\mathrm{ASR}_{\mathrm{K}}\left(2,351 \mathrm{~cm}^{-1}\right)$ is lower than that in AT-ASR $\mathrm{K}$ $\left(2,483 \mathrm{~cm}^{-1}\right)$, though they are similar between 13C-ASR and AT-ASR. We thus assume that the hydrogen bond of the Schiff base is broken in AT-ASR $\mathrm{K}_{\mathrm{K}}$ but not in $13 \mathrm{C}-\mathrm{ASR}_{\mathrm{K}}$. Consequently, the hydrogen-bonding network is destabilized in AT-ASR $\mathrm{K}_{\mathrm{K}}$, and protein structural changes proceed through the network, where the L, M (deprotonation of the Schiff base), and $\mathrm{O}$ states can be produced from AT-ASR. In contrast, structural perturbation of the Schiff base region is smaller in $13 \mathrm{C}-\mathrm{ASR}_{\mathrm{K}}$, where the structural changes are distributed more widely.

The number of observed water bands was two for 13C-ASR and three for AT-ASR (Figure 20). As discussed above, the two water bands in 13C-ASR are assignable to the water molecule in the Schiff base region. The presence of an additional water band indicates involvement of more distant water upon formation of AT-ASR $\mathrm{K}_{\mathrm{K}}$. The second nearest water molecule in the X-ray structure is located $8.3 \AA$ from the Schiff base nitrogen in the structure of AT-ASR and $8.0 \AA$ in the structure of 13C-ASR [16]. That 
water is located between Trp176 and Phe213 in the cytoplasmic region. The third nearest water molecule in the X-ray structure is located $9.2 \AA$ from the Schiff base nitrogen in the structure of AT-ASR and $9.7 \AA$ in the structure of 13C-ASR [16]. That water is located near Arg72 in the extracellular region. No water molecules are present near the polyene chain. Thus, water signals may also be consistent with the above view that the chromophore of $13 \mathrm{C}-\mathrm{ASR}_{\mathrm{K}}$ is distorted more widely along the polyene chain than that of AT-ASR $\mathrm{K}_{\mathrm{K}}$, which has larger changes in the Schiff base region.

In conclusion, ASR accommodates both all-trans and 13-cis, 15-syn retinal in the ground state according to the X-ray crystal structure [16]. On the other hand, the present FTIR study revealed that protein structural changes upon retinal photoisomerization were significantly different between 13C-ASR and AT-ASR. They must trigger global protein structural changes in each photoreaction cycle, resulting in the photochromic behavior. The photochromic signaling mechanism of ASR has not been found, but we should be able to reveal such mechanism if the AT-ASR and 13C-ASR states differ in the binding affinity of the ASRT. The other possibility is that the M state from the photocycle of AT-ASR, which is conformationally changed, would be the signaling state similar to other sensory rhodopsins.

\section{Photochromism of Anabaena Sensory Rhodopsin}

Rhodopsins convert light into signal or energy, and retinal is their chromophore molecule [62-64]. The retinal forms a protonated Schiff base linkage $\left(\mathrm{C}=\mathrm{NH}^{+}\right)$with a lysine at the seventh helix in original state.

It is well-known that the protein environment of rhodopsins accommodates the retinal chromophore optimally to its functions. For example, the specific chromophore-protein interaction leads wide color tuning in human visual pigments with a common chromophore (11-cis retinal) [65], and protein controls the highly efficient photoisomerization from 11-cis to the all-trans form in visual rhodopsins [66].

Specific control of retinal photochemistry by protein can be also seen in rhodopsins from halophilic archaebacteria such as the light-driven proton pump bacteriorhodopsin (BR) $[5,6,66]$. Unlike visual rhodopsins, $\mathrm{BR}$ accommodates the retinal chromophore as the all-trans,15-anti ( $\left.\mathrm{AT} ; \mathrm{BR}_{\mathrm{AT}}\right)$ and 13-cis, 15-syn (13C; $\mathrm{BR}_{13 \mathrm{C}}$ ) forms (Figure 22a) [67]. $\mathrm{BR}_{\mathrm{AT}}$ and $\mathrm{BR}_{13 \mathrm{C}}$ are in equilibrium in the dark, while only $\mathrm{BR}_{\mathrm{AT}}$ possesses proton-pump activity (Figure 22b). Absorption of light by $\mathrm{BR}_{\mathrm{AT}}$ yields isomerization to the 13-cis, 15-anti form, which triggers a cyclic reaction that comprises the series of intermediates, intermediates, $\mathrm{K}, \mathrm{L}, \mathrm{M}, \mathrm{N}$, and $\mathrm{O}$ [5,6]. During the photocycle, one proton is translocated from the cytoplasmic to extracellualr side.

Photoexcitation of $\mathrm{BR}_{13 \mathrm{C}}$ partially converts it to $\mathrm{BR}_{\mathrm{AT}}$, which is called "light-adaptation", but $\mathrm{BR}_{\mathrm{AT}}$ is not converted into $\mathrm{BR}_{13 \mathrm{C}}$ photochemically. Photocycle of $\mathrm{BR}_{\mathrm{AT}}$ with $100 \%$ yield is advantageous for repeating the proton-pumping cycle. This is also the case for other proton pumps found in eubacteria (proteorhodopsin) [14] and eucaryotes (Leptosphaeria rhodopsin) [68]. In addition, haloarchaeal sensory rhodopsins possess only the AT chromophore in the dark, indicating that its photocycle is important also for light-signal conversion [69,70]. Thus, the photocycle of the AT form with $100 \%$ yield has been the common mechanism for the functional processes of microbial rhodopsins. 
Figure 22. (a). The structure of the retinal chromophore of microbial rhodopsins in the dark. (b). Photo and thermal reaction scheme in a light-driven proton pump bacteriorhodopsin (BR). Only $\mathrm{BR}_{\mathrm{AT}}$ possesses proton-pump activity, and the reaction of $\mathrm{BR}_{\mathrm{AT}}$ is $100 \%$ cyclic without any branching reaction into $\mathrm{BR}_{13 \mathrm{C}}$. Dotted arrows represent thermal reaction in the dark. (c). Photo and thermal reaction scheme in Anabaena sensory rhodopsin (ASR). While $\mathrm{ASR}_{\mathrm{AT}}$ is a predominant species in the dark (dotted arrow), photoexcitation of $\mathrm{ASR}_{\mathrm{AT}}$ and $\mathrm{ASR}_{13 \mathrm{C}}$ yields the reaction of each species, either cyclic or branching, leading to the photocycle or photochromism, respectively. $\mathrm{x}$ and $\mathrm{y}$ are the branching ratio from $\mathrm{ASR}_{\mathrm{AT}}$ and $\mathrm{ASR}_{13 \mathrm{C}}$, respectively. These figures are reprinted with permission from Kawanabe et al [71]. Copyright 2007 American Chemical Society.

(a)

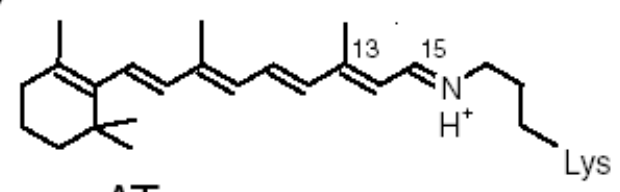

AT (all-trans, 15-anti)

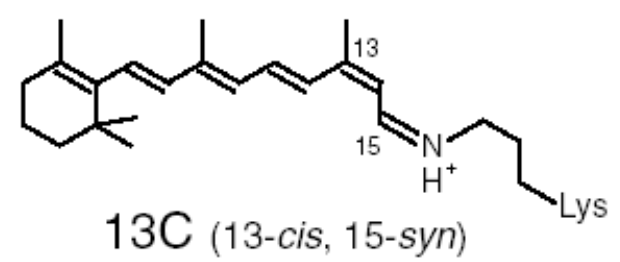

(b)

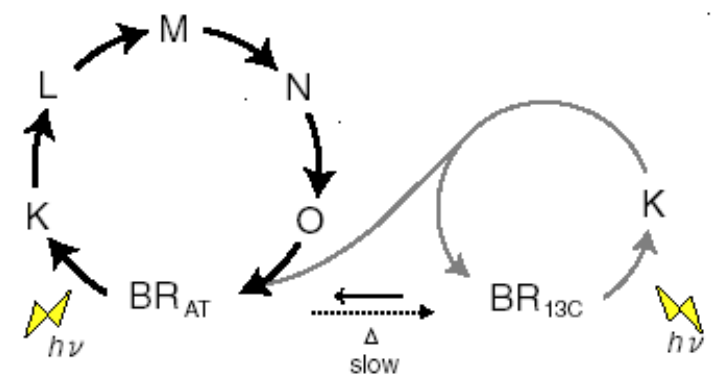

(c)

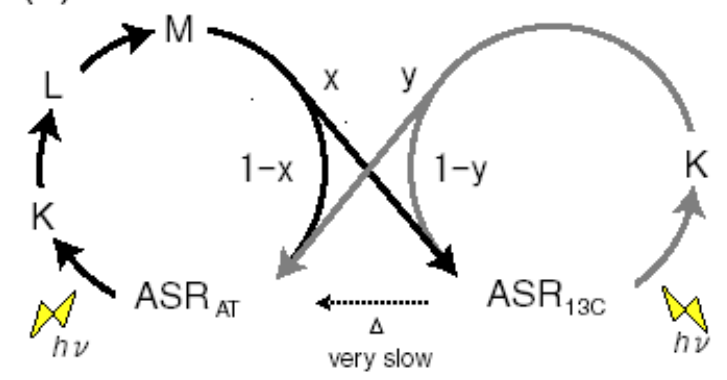

Recently, a microbial rhodopsin has been discovered in Anabaena (Nostoc) PCC7120, which is believed to function as a photoreceptor for chromatic adaptation [15]. In fact, the expected photochromism was found between the AT and 13C forms for Anabaena sensory rhodopsin (ASR) [57]. 
These findings imply strongly branching reactions, from $\mathrm{ASR}_{\mathrm{AT}}$ to $\mathrm{ASR}_{13 \mathrm{C}}$ and from $\mathrm{ASR}_{13 \mathrm{C}}$ to $\mathrm{ASR}_{\mathrm{AT}}$ (Figure 22c), in striking contrast to what is known for microbial rhodopsins. Ideally, the conversion ratios should be unity for photochromic reactions $(x=y=1$ in Figure 22c), but this is exactly the opposite of the properties of pump rhodopsins, such as BR. X-ray crystal structures reported similar chromophore structures and protein environments for $\mathrm{ASR}_{\mathrm{AT}}$ [16] and $\mathrm{BR}_{\mathrm{AT}}$ [7]. Do photochromic reactions indeed take place for $\mathrm{ASR}_{\mathrm{AT}}$ and $\mathrm{ASR}_{13 \mathrm{C}}$ ? In this chapter, we determined the branching ratios ( $\mathrm{x}$ and $\mathrm{y}$ values) for $\mathrm{ASR}_{\mathrm{AT}}$ and $\mathrm{ASR}_{13 \mathrm{C}}$ by means of low-temperature UV-visible spectroscopy. Surprisingly, the obtained $\mathrm{x}$ and $\mathrm{y}$ values were unity, indicating that the photoreactions of $\mathrm{ASR}_{\mathrm{AT}}$ and $\mathrm{ASR}_{13 \mathrm{C}}$ are completely photochromic. The complete photochromic reactions are highly advantageous for the chromatic sensor function of ASR.

\subsection{Photoconversion of $\mathrm{ASR}_{A T}$ (1) Photoreaction at $170 \mathrm{~K}$}

We first examined the branching ratio of $\operatorname{ASR}_{\mathrm{AT}}$ ( $\mathrm{x}$ value in Figure 22c) because previous HPLC analysis revealed that the dark-adapted ASR in PC liposomes contains predominantly (97\%) $\mathrm{ASR}_{\mathrm{AT}}$ [59]. Dark-adapted ASR was illuminated at $170 \mathrm{~K}$, and then warmed to $277 \mathrm{~K}$. The photoconversion yield of $\mathrm{ASR}_{\mathrm{AT}}$ to its intermediates was calculated using the spectra at $170 \mathrm{~K}$, which was compared with the conversion of $\mathrm{ASR}_{\mathrm{AT}}$ to $\mathrm{ASR}_{13 \mathrm{C}}$ at $277 \mathrm{~K}$.

The black line in Figure 23a shows the absorption spectrum of the dark-adapted ASR at $170 \mathrm{~K}$ $\left(\lambda_{\max }=554 \mathrm{~nm}\right.$ ). Illumination at $>580 \mathrm{~nm}$ (red line) or $501 \mathrm{~nm}$ (blue line) resulted in reduction of the peak absorbance and increase of the shorter or longer wavelength tail, indicating the formation of the $\mathrm{L}$ and $\mathrm{K}$ photointermediates, respectively. Figure $23 \mathrm{~b}$ shows the corresponding difference spectra, and positive peaks at 474 and $605 \mathrm{~nm}$ are characteristic absorption of the $\mathrm{L}$ and $\mathrm{K}$ intermediates, respectively. On the other hand, no positive band at about $400 \mathrm{~nm}$ indicates that the $\mathrm{M}$ intermediate is not formed at $170 \mathrm{~K}$.

Since the red and blue spectra in Figure $23 \mathrm{~b}$ contain contribution of the $\mathrm{L}$ and $\mathrm{K}$ intermediates, we next obtained the $\mathrm{K}$ minus $\mathrm{ASR}_{\mathrm{AT}}$ and $\mathrm{L}$ minus $\mathrm{ASR}_{\mathrm{AT}}$ spectra. The $\mathrm{L}$ minus $\mathrm{ASR}_{\mathrm{AT}}$ spectrum was obtained by subtracting the blue spectrum from the red one in Figure 23b, so that the spectral shape at about $600 \mathrm{~nm}$ coincides with that of the absolute spectrum of the dark-adapted ASR (black line in Figure 23a). The red spectrum in Figure 23c represents the L minus ASR $_{\mathrm{AT}}$ spectrum thus obtained. Then, the $\mathrm{L}$ minus $\mathrm{ASR}_{\mathrm{AT}}$ spectrum was subtracted from the blue spectrum in Figure $23 \mathrm{~b}$ so as to resemble that at $130 \mathrm{~K}$ (black dotted line in Figure 23c), where the only photoproduct is the $\mathrm{K}$ intermediate. The blue spectrum in Figure $23 \mathrm{c}$ represents the resulting $\mathrm{K}$ minus $\mathrm{ASR}_{\mathrm{AT}}$ spectrum. Isosbestic points are at $520 \mathrm{~nm}$ between $\mathrm{ASR}_{\mathrm{AT}}$ and $\mathrm{L}$, and at $575 \mathrm{~nm}$ between $\mathrm{ASR}_{\mathrm{AT}}$ and $\mathrm{K}$.

We then determined the absorption spectra of the $\mathrm{K}$ and $\mathrm{L}$ intermediates of $\mathrm{ASR}_{\mathrm{AT}}$ at $170 \mathrm{~K}$. Absorption spectrum of the $K$ intermediate can be obtained from the $K$ minus ASR $_{\mathrm{AT}}$ difference spectrum (blue line in Figure 23c) and photoconversion ratio from $\mathrm{ASR}_{\mathrm{AT}}$ to the $\mathrm{K}$ intermediate. Absorption spectrum of the $\mathrm{L}$ intermediate can be obtained from the $\mathrm{L}$ minus $\mathrm{ASR}_{\mathrm{AT}}$ difference spectrum (red line in Figure 23c) and photoconversion ratio from $\mathrm{ASR}_{\mathrm{AT}}$ to the $\mathrm{L}$ intermediate. Five colored lines in Figures $24 \mathrm{a}$ or b correspond to the calculated spectra of the K intermediate of $\mathrm{ASR}_{\mathrm{AT}}$ or the $\mathrm{L}$ intermediate of $\mathrm{ASR}_{\mathrm{AT}}$ at various percentages of conversion (100-17\% from orange to blue; $32 \%$ for the red line, respectively). 
Figure 23. (Left). (a). Absorption spectra of the dark-adapted ASR (black line), illuminated with >580 $\mathrm{nm}$ (red line) and $501 \mathrm{~nm}$ (blue line) light at $170 \mathrm{~K}$. It should be noted that the dark-adapted ASR corresponds to $\mathrm{ASR}_{\mathrm{AT}}$, because it contains negligible amount of $\mathrm{ASR}_{13 \mathrm{C}}$ $(2.9 \%)$ in the present sample conditions [59]. (b). Light-minus-dark difference absorption spectra of ASR with $>580 \mathrm{~nm}$ (red line) and $501 \mathrm{~nm}$ (blue line) light at $170 \mathrm{~K}$. (c). L minus $\mathrm{ASR}_{\mathrm{AT}}$ (red line) and $\mathrm{K}$ minus $\mathrm{ASR}_{\mathrm{AT}}$ (blue line) difference absorption spectra at $170 \mathrm{~K}$. Black broken line corresponds to the $\mathrm{K}$ minus $\mathrm{ASR}_{\mathrm{AT}}$ spectrum at $130 \mathrm{~K}$, where only the $\mathrm{K}$ intermediate is formed. See text for detail. One division of the y-axis corresponds to 0.1(a) and $0.05(b, c)$ absorbance units. These figures are reprinted with permission from Kawanabe et al. [71]. Copyright 2007 American Chemical Society.

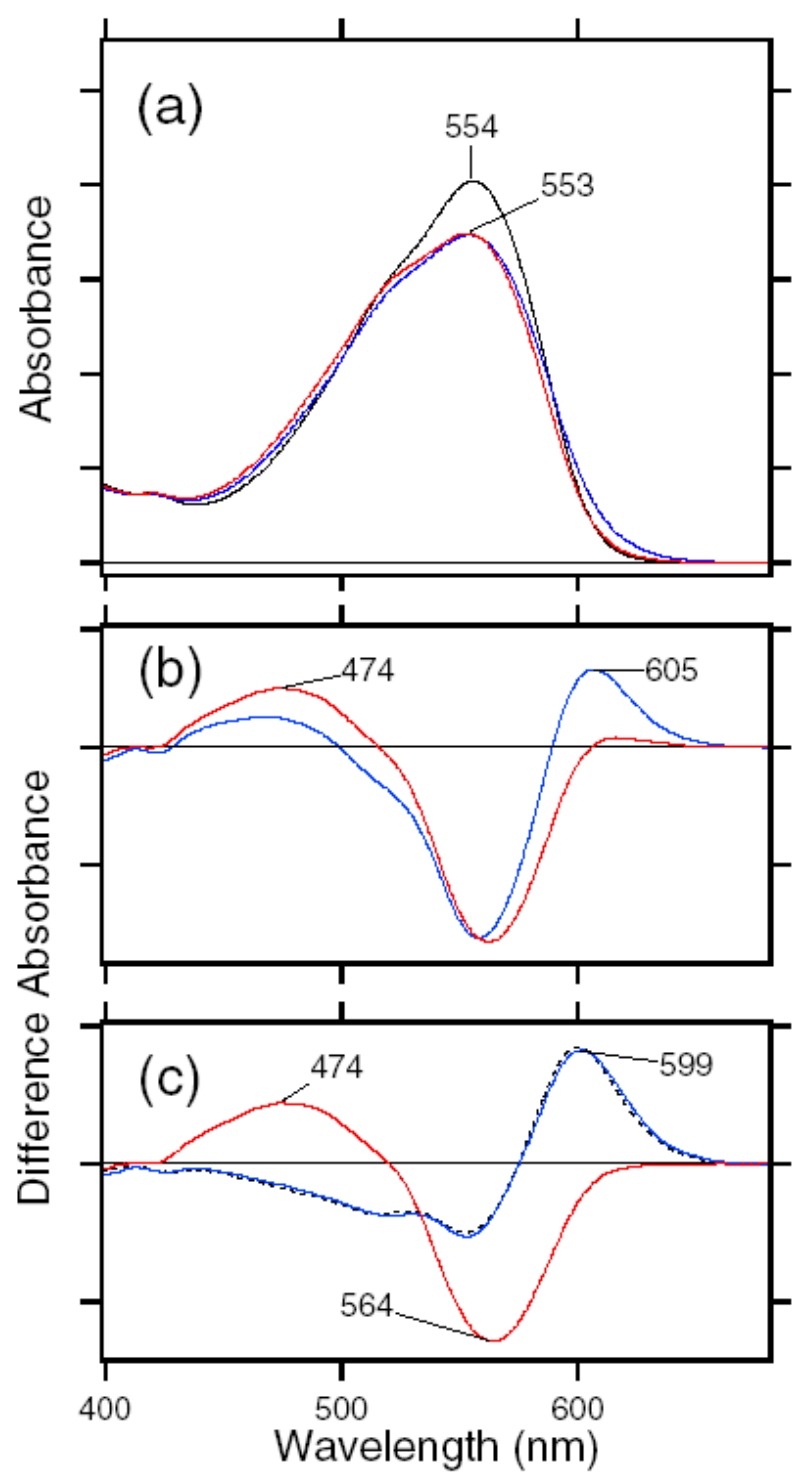


Figure 24. Determination of the absorption spectra of the $\mathrm{K}$ and $\mathrm{L}$ intermediates of $\mathrm{ASR}_{\mathrm{AT}}$ at $170 \mathrm{~K}$. (a,b) Black solid line represents absorption spectrum of $\mathrm{ASR}_{\mathrm{AT}}$ at $170 \mathrm{~K}$. (c) Second derivatives of absorption spectra in Figure 24b, where the corresponding spectra are shown by the same color. (d). Absorption spectra of $\mathrm{ASR}_{\mathrm{AT}}$ (black line), the $\mathrm{K}$ intermediate (blue line) and the $\mathrm{L}$ intermediate (red line) at $170 \mathrm{~K}$. The spectra of the $\mathrm{K}$ and $\mathrm{L}$ intermediates are reproduced from the red spectra in Figure $24 \mathrm{a}$ and $\mathrm{b}$, respectively. One division of the y-axis corresponds to $0.1(\mathrm{a}, \mathrm{b}, \mathrm{d})$ and 0.0002 (c) absorbance units. These figures are reprinted with permission from Kawanabe et al. [71]. Copyright 2007 American Chemical Society.

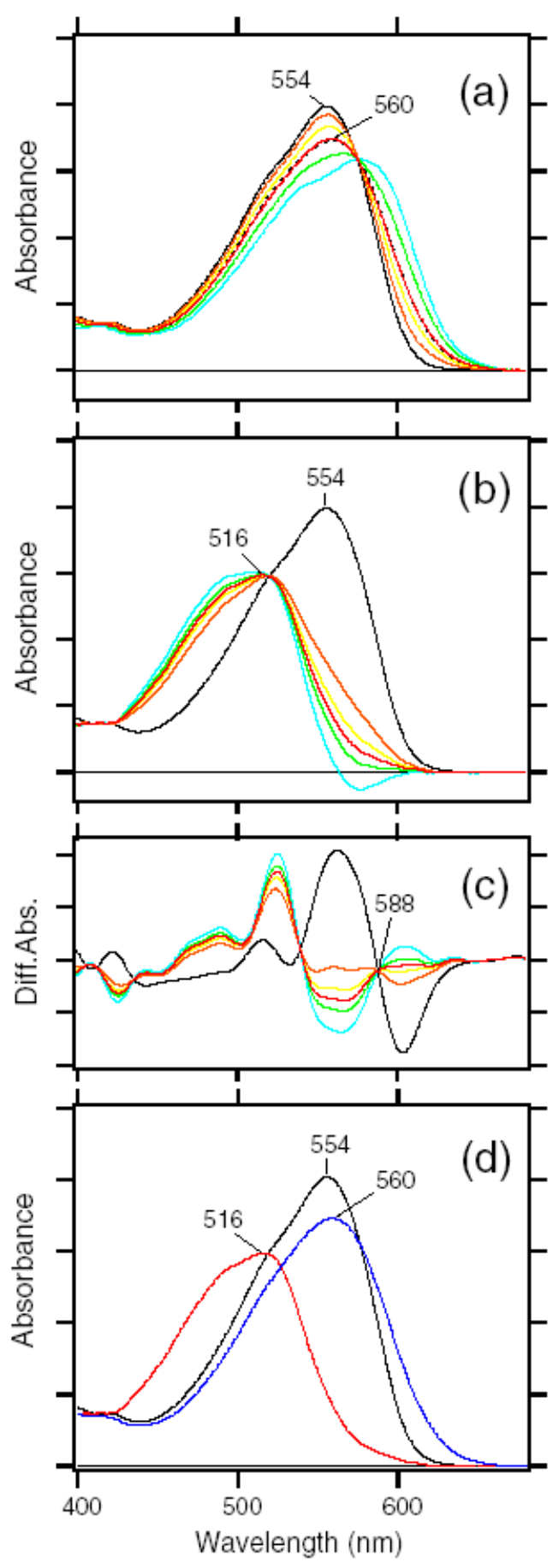


The broken black line in Figure 24a represents the absorption spectrum of the $\mathrm{K}$ intermediate of $\mathrm{ASR}_{\mathrm{AT}}$ at $130 \mathrm{~K}$, which was determined by illuminating $\mathrm{ASR}_{\mathrm{AT}}$ at two wavelengths as described in Figure 25. Since the red spectrum in Figure 24a coincided well with the black broken line, we regard the red one as the absorption spectrum of the $\mathrm{K}$ intermediate of $\mathrm{ASR}_{\mathrm{AT}}$ at $170 \mathrm{~K}$. On the other hand, the absorption spectrum of the $\mathrm{L}$ intermediate was determined from the spectral analysis of the second derivatives of the absorption spectra in Figure 24b. The second derivatives in Figure $24 \mathrm{c}$ show that the red spectrum coincides with the zero line at $>588 \mathrm{~nm}$. We assume that the $\mathrm{L}$ intermediate does not contain spectral component in the second derivative at $>588 \mathrm{~nm}$. Consequently, we regarded the red one in Figure $24 \mathrm{~b}$ as the absorption spectrum of the $\mathrm{L}$ intermediate of $\mathrm{ASR}_{\mathrm{AT}}$ at $170 \mathrm{~K}$. Blue and red spectra in Figure 24d correspond to the absolute spectra of the $\mathrm{K}$ and $\mathrm{L}$ intermediates, respectively.

Figure 25 shows the determination of the absorption spectra of the $\mathrm{K}$ intermediates of $\mathrm{ASR}_{\mathrm{AT}}$ and $\mathrm{ASR}_{13 \mathrm{C}}$ at $130 \mathrm{~K}$. These spectra were calculated for each illumination wavelengths from the spectra in Figure $25 \mathrm{c}$ and $\mathrm{d}$ by taking account of the isomeric compositions of the dark-adapted $\left(97 \% \mathrm{ASR}_{\mathrm{AT}}\right.$ and $\left.3 \% \mathrm{ASR}_{13 \mathrm{C}}\right)$ and light-adapted $\left(22 \% \mathrm{ASR}_{\mathrm{AT}}\right.$ and $\left.78 \% \mathrm{ASR}_{13 \mathrm{C}}\right) \mathrm{ASR}$. The almost identical spectra in Figure $25 \mathrm{c}$ and e indicate that the dark-adapted state can be regarded as $\mathrm{ASR}_{\mathrm{AT}}$. In Figure $25 \mathrm{~g}$ to determine absorption spectrum of an intermediate, the photoconversion ratio from the unphotolyzed state to the intermediate must be obtained. Such a ratio can be obtained by illuminations at two wavelengths if the quantum yields are independent of wavelength [71]. For instance, $\mathrm{ASR}_{\mathrm{AT}}$ is illuminated at $480 \mathrm{~nm}$ or $577 \mathrm{~nm}$ under photoequilibrium conditions:

$$
\begin{aligned}
& \left(1-\mathrm{x}_{1}\right) \operatorname{Abs}\left(\operatorname{ASR}_{\mathrm{AT}}, 480 \mathrm{~nm}\right)=\mathrm{x}_{1} \phi \operatorname{Abs}\left(\operatorname{ASR}_{\mathrm{AT}}(\mathrm{K}), 480 \mathrm{~nm}\right) \\
& \left(1-\mathrm{x}_{2}\right) \operatorname{Abs}\left(\operatorname{ASR}_{\mathrm{AT}}, 577 \mathrm{~nm}\right)=\mathrm{x}_{2} \phi \operatorname{Abs}\left(\operatorname{ASR}_{\mathrm{AT}}(\mathrm{K}), 577 \mathrm{~nm}\right)
\end{aligned}
$$

where $\mathrm{x}_{1}$ and $\mathrm{x}_{2}$ are relative amount of $\operatorname{ASR}_{\mathrm{AT}}(\mathrm{K})$ in the photosteady state mixture, $\phi$ is the relative quantum yield of $\operatorname{ASR}_{\mathrm{AT}}(\mathrm{K})$ to $\mathrm{ASR}_{\mathrm{AT}}$. $\operatorname{Abs}\left(\mathrm{ASR}_{\mathrm{AT}}, 480 \mathrm{~nm}\right)$ and $\operatorname{Abs}\left(\mathrm{ASR}_{\mathrm{AT}}(\mathrm{K}), 480 \mathrm{~nm}\right)$ are the absorbance of $\mathrm{ASR}_{\mathrm{AT}}$ and the $\mathrm{K}$ intermediate at $480 \mathrm{~nm}$, respectively. On the other hand, the following equations are derived from the difference spectra before and after illumination:

$$
\begin{aligned}
& \Delta \operatorname{Abs}(480 \mathrm{~nm})=\mathrm{x}_{1}\left(\operatorname{Abs}\left(\operatorname{ASR}_{\mathrm{AT}}(\mathrm{K}), 480 \mathrm{~nm}\right)-\operatorname{Abs}_{\left.\left(\operatorname{ASR}_{\mathrm{AT}}, 480 \mathrm{~nm}\right)\right)}\right. \\
& \Delta \operatorname{Abs}(577 \mathrm{~nm})=\mathrm{x}_{2}\left(\operatorname{Abs}\left(\operatorname{ASR}_{\mathrm{AT}}(\mathrm{K}), 577 \mathrm{~nm}\right)-\operatorname{Abs}_{\left.\left(\mathrm{ASR}_{\mathrm{AT}}, 577 \mathrm{~nm}\right)\right)}\right.
\end{aligned}
$$

where $\Delta \operatorname{Abs}(480 \mathrm{~nm})$ and $\Delta \operatorname{Abs}(577 \mathrm{~nm})$ are the difference absorbances at 480 and $577 \mathrm{~nm}$, respectively. From the blue $(480 \mathrm{~nm})$ and red $(577 \mathrm{~nm})$ spectra in Figure $25 \mathrm{e}$, absorption spectrum of the $\mathrm{K}$ intermediate of $\mathrm{ASR}_{\mathrm{AT}}$ can be determined by obtaining $\mathrm{x}_{1}$ and $\mathrm{x}_{2}$ values (red solid line in Figure $25 \mathrm{~g}$ ). Red dotted line corresponds to the spectrum of the $\mathrm{K}$ intermediate of $\mathrm{ASR}_{\mathrm{AT}}$ obtained from the green $(548 \mathrm{~nm})$ and red $(577 \mathrm{~nm})$ spectra in Figure 25e. Red solid and dotted spectra in Figure $25 \mathrm{~g}$ are almost identical, implying that quantum yields are wavelength independent. 
Figure 25. Determination of the absorption spectra of the $K$ intermediates of $\operatorname{ASR}_{\mathrm{AT}}$ and $\mathrm{ASR}_{13 \mathrm{C}}$ at $130 \mathrm{~K}$. Black solid line represents the absorption spectra of the dark-adapted (a) and light-adapted (b) ASR at $130 \mathrm{~K}$. Blue, green, and red spectra are those by illumination with a 480, 548, and $577 \mathrm{~nm}$ light through interference filters (sharp colored peaks in the figure), respectively. (c) and (d). Light-minus-dark difference absorption spectra of the dark-adapted (c) and light-adapted (d) ASR by illumination with 480 (blue), 548 (green), and 577 (red) nm lights at $130 \mathrm{~K}$. (e) and (f). K-minus-ASR AT $_{\text {(e) and K-minus-ASR }}$ an $_{\text {(f) }}$ difference absorption spectra by illumination with 480 (blue), 548 (green), and 577 (red) nm lights at $130 \mathrm{~K}$. Blue solid, dotted, and broken lines represent the spectra of the $\mathrm{K}$ intermediate of $\mathrm{ASR}_{13 \mathrm{C}}$ obtained from the blue and red, green and red, and blue and green spectra in $\mathrm{f}$. We regarded the red and blue solid lines as the spectra of the $\mathrm{K}$ intermediates of $\mathrm{ASR}_{\mathrm{AT}}$ and $\mathrm{ASR}_{13 \mathrm{C}}$, respectively. One division of the $\mathrm{y}$-axis corresponds to $0.2(\mathrm{a}, \mathrm{b})$, $0.04(\mathrm{c}, \mathrm{d}, \mathrm{e}, \mathrm{f})$ and $0.1(\mathrm{~g})$ absorbance units. These figures are reprinted with permission from Kawanabe et al. [71]. Copyright 2007 American Chemical Society.

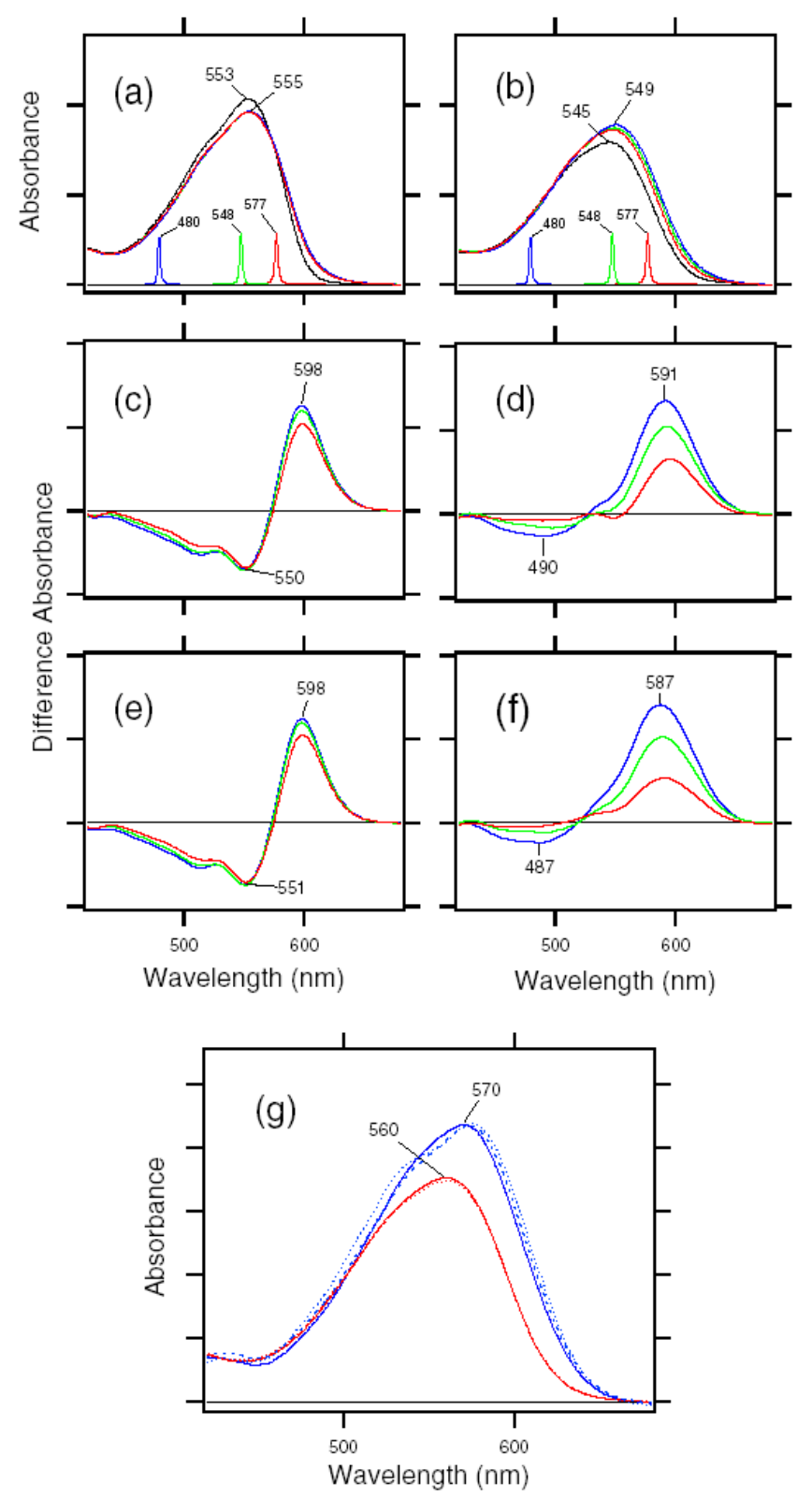


4.2. Photoconversion of $A S R_{A T}$ (2) Thermal Relaxation by warming the Sample from $170 \mathrm{~K}$ to $277 \mathrm{~K}$

We reconstituted the experimentally obtained spectra (dotted black lines in Figures 26a and c) by use of the spectra in Figure 24d. For the illumination at $>580 \mathrm{~nm}$, the dotted black spectrum in Figure 26a coincides well with the sum of $78 \%$ ASR, 5\% K, and 17\% L (green line in Figure 26a), indicating the $22( \pm 2) \%$ conversion to intermediates at $170 \mathrm{~K}$. On the other hand, for the illumination at $501 \mathrm{~nm}$, the dotted black spectrum in Figure 26c is well coincident with the sum of $68 \%$ ASR, $18 \% \mathrm{~K}$, and $14 \% \mathrm{~L}$ (green line in Figure 26c), indicating the $32( \pm 5) \%$ conversion at $170 \mathrm{~K}$. We then warmed these states from 170 to $277 \mathrm{~K}$ so as to complete the thermal reactions of the $\mathrm{K}$ and $\mathrm{L}$ states to their end products, and calculated the conversion yield from the spectra. Dotted black lines in Figures $26 \mathrm{~b}$ and d represent the spectra at $277 \mathrm{~K}$ after illumination at $>580$ and $501 \mathrm{~nm}$, respectively, at $170 \mathrm{~K}$.

Figure 26. (a) and (c). Absorption spectra of the dark-adapted ASR before (black solid lines) and after (black dotted lines) illuminations with $>580 \mathrm{~nm}$ (a) or $501 \mathrm{~nm}$ (c) light at $170 \mathrm{~K}$. Green lines represent the spectra reconstituted using those in Figure 24d. Under the present illumination conditions, $22( \pm 2)$ and $32( \pm 5) \%$ portion were converted into the intermediates in a and c, respectively. (b) and (d). Black solid lines represent absorption spectra of the dark-adapted ASR at $277 \mathrm{~K}$. Red and blue lines correspond to the calculated absorption spectra of $\mathrm{ASR}_{\mathrm{AT}}$ and $\mathrm{ASR}_{13 \mathrm{C}}$ at $277 \mathrm{~K}$, respectively, which were obtained from those of dark- and light-adapted ASR and the HPLC analysis [59]. Black dotted lines represent absorption spectra at $277 \mathrm{~K}$ after illuminations with $>580 \mathrm{~nm}$ (b) or $501 \mathrm{~nm}$ (d) light at $170 \mathrm{~K}$. Under the present illumination conditions, $23( \pm 2)$ and $34( \pm 4) \%$ portion were converted from $\mathrm{ASR}_{\mathrm{AT}}$ to $\mathrm{ASR}_{13 \mathrm{C}}$ at $277 \mathrm{~K}$ in $\mathrm{b}$ and $\mathrm{d}$, respectively. From a-d, the branching ratio ( $\mathrm{x}$ in Figure 22c) is obtained to be $1.02 \pm 0.13$ and $1.10 \pm 0.09$ for illuminations at $>580 \mathrm{~nm}$ and $501 \mathrm{~nm}$, respectively, indicating complete branching reactions from $\mathrm{ASR}_{\mathrm{AT}}$ for both illumination conditions. One division of the y-axis corresponds to 0.1 absorbance units. These figures are reprinted with permission from Kawanabe et al. [71]. Copyright 2007 American Chemical Society.

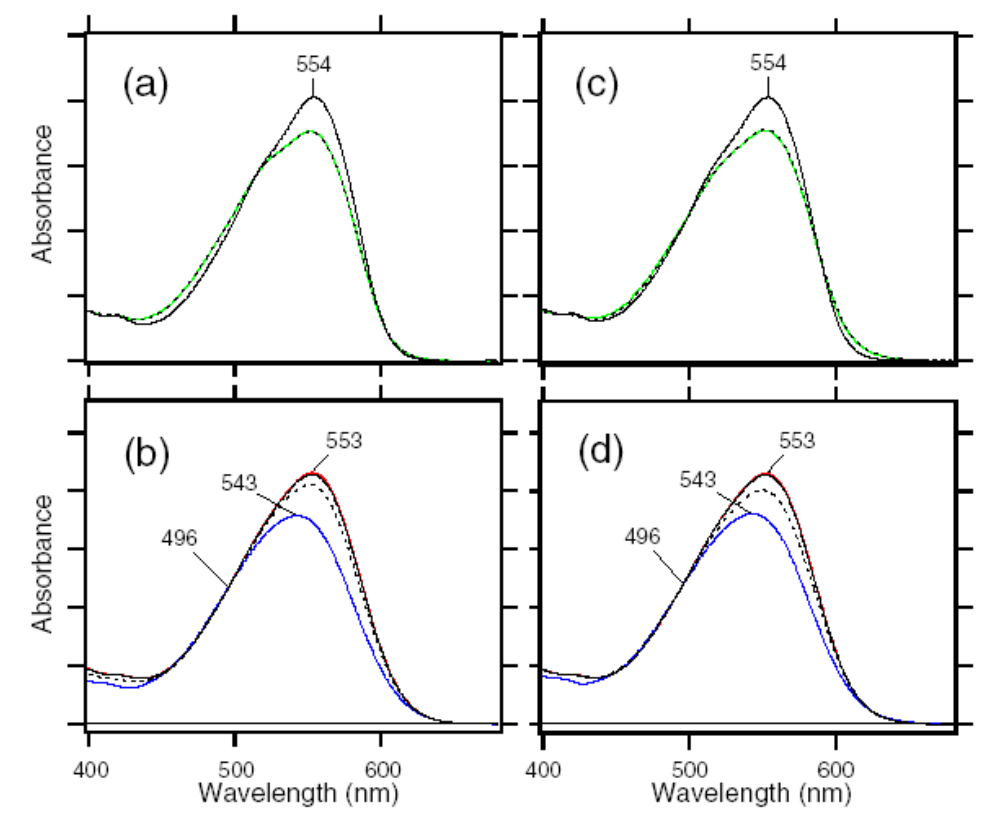


Using the absorption spectra of $\mathrm{ASR}_{\mathrm{AT}}$ and $\mathrm{ASR}_{13 \mathrm{C}}$, the percentages of conversion were calculated to be $23( \pm 2) \%$ and $34( \pm 4) \%$ in Figures $26 \mathrm{~b}$ and d, respectively. The branching ratios ( $\mathrm{x}$ in Figure 22) were thus determined to be $1.02 \pm 0.13$ and $1.10 \pm 0.09$ for illuminations at >580 and $501 \mathrm{~nm}$, respectively. These values demonstrate that the $\mathrm{K}$ and $\mathrm{L}$ intermediates formed from $\mathrm{ASR}_{\mathrm{AT}}$ are completely converted into $\mathrm{ASR}_{13 \mathrm{C}}$ without regaining the initial state in a photocyclic reaction.

By means of low-temperature FTIR spectroscopy, we had previously suggested that the primary photoproduct of $\mathrm{ASR}_{\mathrm{AT}}$ is the 13-cis, 15-anti form as in BR (Figure 27) [20]. In BR, thermal isomerization takes place at the $\mathrm{C} 13=\mathrm{C} 14$ bond with virtually $100 \%$ yield, recovering the original AT state. In contrast, in ASR thermal isomerization is likely to occur at the $\mathrm{C} 15=\mathrm{N}$ bond following photoisomerization of $\mathrm{ASR}_{\mathrm{AT}}$, which converts to the $13 \mathrm{C}$ state with $100 \%$ yield (Figure 27).

Figure 27. Structural changes of the all-trans, 15-anti chromophore during photoreactions.

The all-trans, 15-anti form, either in $\mathrm{BR}_{\mathrm{AT}}$ or $\mathrm{ASR}_{\mathrm{AT}}$, is first photoconverted to the 13-cis, 15-anti form, followed by thermal isomerization at $\mathrm{C} 13=\mathrm{C} 14$ or $\mathrm{C} 15=\mathrm{N}$ position in $\mathrm{BR}$ or ASR, respectively. Such thermal relaxations lead to $100 \%$ photocyclic and photochromic reactions for $\mathrm{BR}_{\mathrm{AT}}$ and $\mathrm{ASR}_{\mathrm{AT}}$, respectively. This figure is reprinted with permission from Kawanabe et al. [71]. Copyright 2007 American Chemical Society.

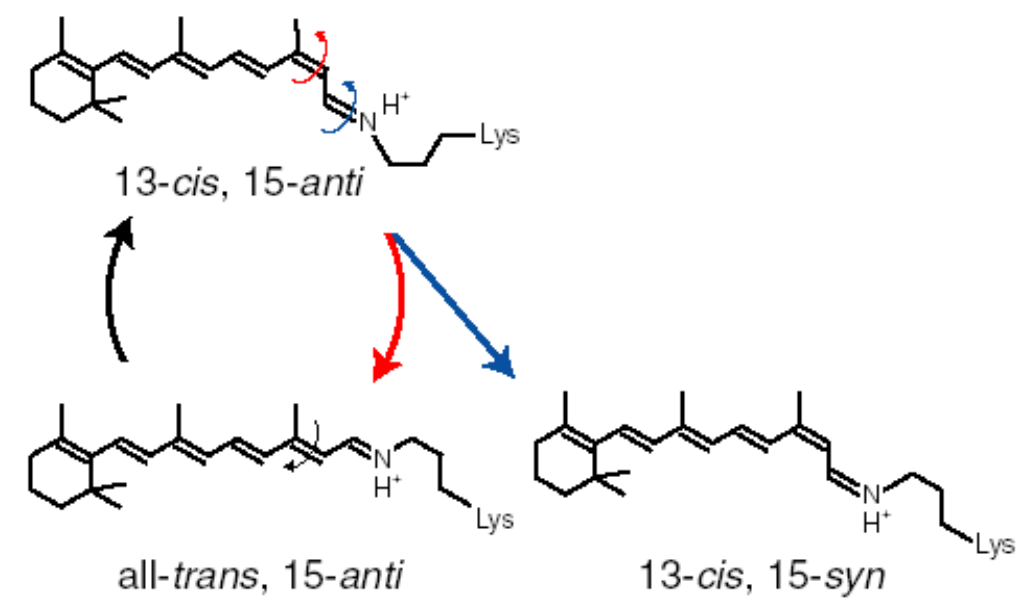

\subsection{Photoconversion of $A S R_{13 C}$ (1) Relative Photoconversion Yields of $A S R_{A T}$ and $A S R_{13 C}$ at $277 \mathrm{~K}$}

What is the branching ratio (y value in Figure 22c) from $\mathrm{ASR}_{13 \mathrm{C}}$ ? Unlike $\mathrm{ASR}_{\mathrm{AT}}$ that is present as nearly the only state in dark-adapted ASR, $\mathrm{ASR}_{13 \mathrm{C}}$ is present in a mixture with $\mathrm{ASR}_{\mathrm{AT}}$. Therefore, we attempted to determine the branching ratio on the basis of relative photoconversion yields. A previous study showed that the dark-adapted or light-adapted ASRs in PC liposomes possess $97.1 \% \mathrm{ASR}_{\mathrm{AT}}$ and $2.9 \% \mathrm{ASR}_{13 \mathrm{C}}$ or $22.1 \% \mathrm{ASR}_{\mathrm{AT}}$ and $77.9 \% \mathrm{ASR}_{13 \mathrm{C}}$, respectively [59]. Since $\mathrm{ASR}_{\mathrm{AT}}$ has greater extinction than ASR $_{13 \mathrm{C}}$ at $500-600 \mathrm{~nm}$ (Figures $26 \mathrm{~b}$ and d), illumination of the dark-adapted ASR yields an absorption decrease in this wavelength region. In contrast, illumination of light-adapted ASR results in the increase of absorption at $500-600 \mathrm{~nm}$, as reported previously [16]. The isosbestic point of ASR $_{\mathrm{AT}}$ and $\mathrm{ASR}_{13 \mathrm{C}}$ is located at $496 \mathrm{~nm}$ (Figures $26 \mathrm{~b}$ and d). 
In Figure 28, we illuminated the dark-adapted and light-adapted ASR with a $496 \mathrm{~nm}$ light at $277 \mathrm{~K}$, and the changes in absorbance at $569 \mathrm{~nm}$ (difference absorption maximum between $\mathrm{ASR}_{\mathrm{AT}}$ and $\mathrm{ASR}_{13 \mathrm{C}}$ at $277 \mathrm{~K}$ ) were plotted as the function of illumination time.

Figure 28. Absorption changes at $569 \mathrm{~nm}$ of the dark-adapted (open circles) and lightadapted (open squares) ASR after illumination at the isosbestic point of $\mathrm{ASR}_{\mathrm{AT}}$ and $\operatorname{ASR}_{13 \mathrm{C}}(496 \mathrm{~nm})$ at $277 \mathrm{~K}$. Absorption changes were fitted by single exponentials (solid and broken lines), and the ratio of the initial slope (light-adapted ASR/dark-adapted ASR) was $0.40: 1$. By taking into accounts of the contents of $\mathrm{ASR}_{\mathrm{AT}}$ and $\mathrm{ASR}_{13 \mathrm{C}}$ in each state, the ratio between $\mathrm{ASR}_{13 \mathrm{C}}$-to-ASR $\mathrm{AT}_{\mathrm{AT}}$ and $\mathrm{ASR}_{\mathrm{AT}}$-to- $\mathrm{ASR}_{13 \mathrm{C}}$ was determined to be $0.77( \pm 0.04)$. This figure is reprinted with permission from Kawanabe et al. [71]. Copyright 2007 American Chemical Society.

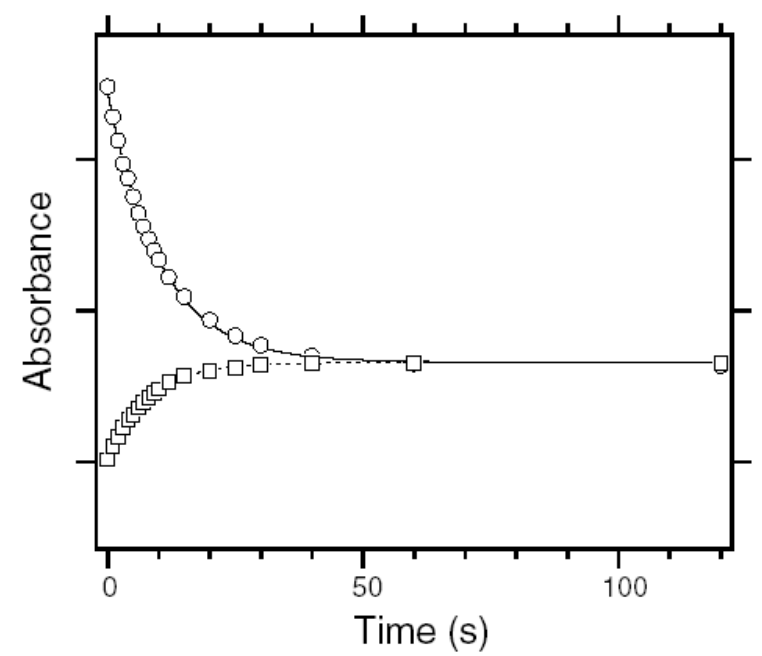

Thermal conversion from $\mathrm{ASR}_{13 \mathrm{C}}$ to $\mathrm{ASR}_{\mathrm{AT}}$ is negligible, because it takes $90 \mathrm{~min}\left(\tau_{1 / 2}\right)$ for $\mathrm{ASR}$ in PC liposomes at $277 \mathrm{~K}$ (data not shown). Absorbance at $569 \mathrm{~nm}$ decreases and increases for the dark-adapted and light-adapted ASR, respectively, and both curves eventually coincide after long illumination (Figure 28). The time courses are well fitted by single exponentials, and each photoconversion yield can be obtained from the initial slope $(t=0)$. By taking into account the contents of $\mathrm{ASR}_{\mathrm{AT}}$ and $\mathrm{ASR}_{13 \mathrm{C}}$ in the dark-adapted and light-adapted forms, we determined the photoconversion yields ratio between $\mathrm{ASR}_{13 \mathrm{C}}$-to- $\mathrm{ASR}_{\mathrm{AT}}$ and $\mathrm{ASR}_{\mathrm{AT}}$-to- $\mathrm{ASR}_{13 \mathrm{C}}$ to be $0.77( \pm 0.04)$ :1. [Sineshchekov et al. estimated a similar photoconversion yield to be $0.3: 1$ from the HPLC analysis of the photosteady state mixture with white or $>520-\mathrm{nm}$ light illumination [57]. While the accurate photoconversion yield is determined by the present method (from the initial slope after illumination at their isosbestic point), such a big difference $(0.77$ vs. 0.3$)$ should be explained. We confirmed that the spectral analysis of the photosteady state, not initial slope, in Figure 28 yields the ratio to be similar (0.75:1). On the other

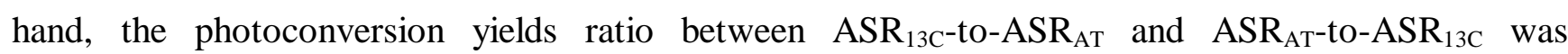
significantly reduced by illumination at longer wavelengths, which is close to the value reported by Sineshchekov et al. [57]. Thus, the photoconversion yields ratio depends on the illumination wavelength; 0.77 by the $496 \mathrm{~nm}$ illumination and about 0.3 by the illumination at $>520 \mathrm{~nm}$. We infer that under the photostationary conditions at $>520 \mathrm{~nm}$, the intermediate state of ASR $_{13 \mathrm{C}}$ is photoexcited, 
presumably forming the original $\mathrm{ASR}_{13 \mathrm{C}}$, while that of $\mathrm{ASR}_{\mathrm{AT}}$ (the $\mathrm{M}$ state) is not. Consequently, $\mathrm{ASR}_{13 \mathrm{C}}$ is accumulated, and the photoconversion yields ratio between $\mathrm{ASR}_{13 \mathrm{C}}$-to-ASR $\mathrm{AT}_{\mathrm{AT}}$ and $\mathrm{ASR}_{\mathrm{AT}}$-to-ASR $\mathrm{ABC}_{13 \mathrm{C}}$ is apparently lowered.] Since the sample is illuminated at the isosbestic point, the ratio is directly correlated with the relative photoconversion yields. Although this value apparently shows a lower branching ratio for $\operatorname{ASR}_{13 \mathrm{C}}$ than for $\operatorname{ASR}_{\mathrm{AT}}(\mathrm{x}=1)$, it should be noted that the photoisomerization quantum yields are not taken into account in this estimate. A lower photoisomerization quantum yield of $\mathrm{ASR}_{13 \mathrm{C}}$ may provide a lower value for $\mathrm{ASR}_{13 \mathrm{C}}$, and it was indeed the case as shown below.

\subsection{Photoconversion of $A S R_{13 C}$ (2) Relative Photoisomerization Quantum Yields of $A S R_{A T}$ and $A S R_{13 C}$} at $130 \mathrm{~K}$

We next compared the relative quantum yields for the photoisomerization of $\mathrm{ASR}_{\mathrm{AT}}$ and $\mathrm{ASR}_{13 \mathrm{C}}$ by comparing the formation of their $\mathrm{K}$ intermediates at $130 \mathrm{~K}$. Since the molar extinction coefficients of their $\mathrm{K}$ intermediates are required for the calculation, we determined the absorption spectra of the $\mathrm{K}$-intermediates of $\mathrm{ASR}_{\mathrm{AT}}$ and $\mathrm{ASR}_{13 \mathrm{C}}$ according to the procedure in Figure 25.

Figure 29. (a). Red and blue broken lines correspond to the absorption spectra of ASR $_{\mathrm{AT}}$ and $\mathrm{ASR}_{13 \mathrm{C}}$ at $130 \mathrm{~K}$, respectively, which were obtained from those of the dark- and lightadapted ASR and the HPLC analysis [59]. Isosbestic point is located at $480 \mathrm{~nm}$. Red and blue solid lines represent absorption spectra of the $\mathrm{K}$ intermediates of $\mathrm{ASR}_{\mathrm{AT}}$ and $\mathrm{ASR}_{13 \mathrm{C}}$ at $130 \mathrm{~K}$, which were obtained according to the procedure in Figure 25. (b). Time-dependent absorbance changes of the dark-adapted (open circles) and light-adapted (open squares) ASR. Each sample was illuminated at the isosbestic point at $130 \mathrm{~K}(480 \mathrm{~nm}$; a), and absorbance changes were monitored at 596 and $590 \mathrm{~nm}$ for the dark-adapted and light-adapted ASR, respectively. These figures are reprinted with permission from Kawanabe et al. [71]. Copyright 2007 American Chemical Society.
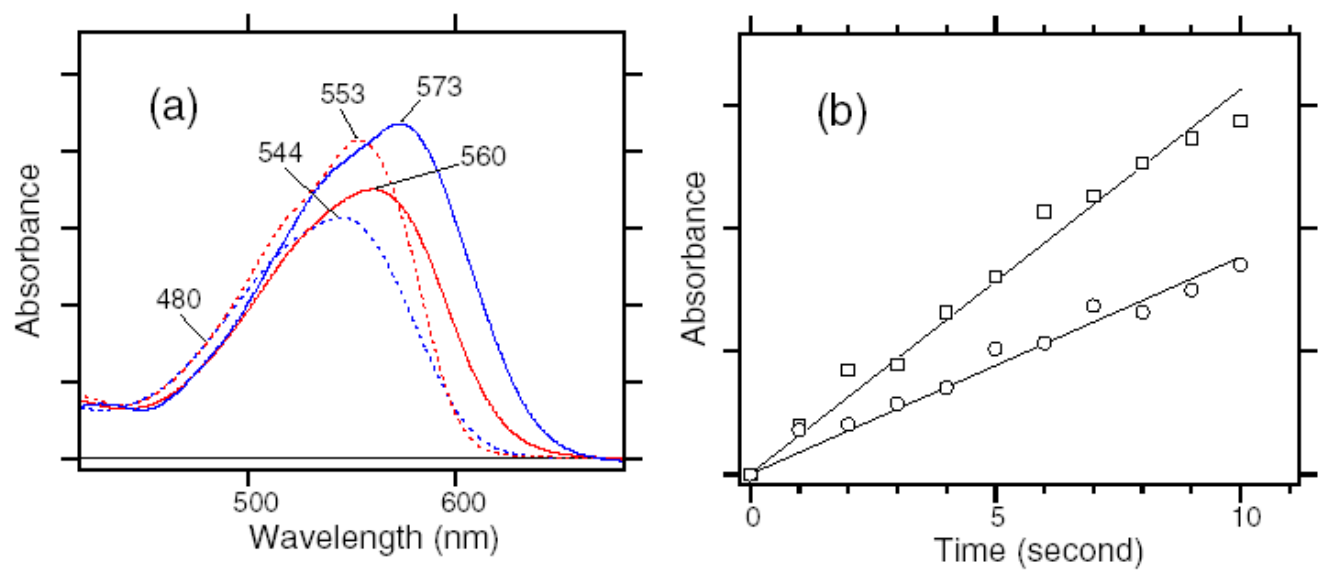

Solid red and blue lines in Figure 29a represent absorption spectra of the $\mathrm{K}$ intermediates of ASR $\mathrm{AT}_{\mathrm{A}}$ and $\mathrm{ASR}_{13 \mathrm{C}}$, respectively. Interestingly, the absorption of the $\mathrm{K}$ state is decreased for $\mathrm{ASR}_{\mathrm{AT}}$ but increased for $\mathrm{ASR}_{13 \mathrm{C}}$ increased. Together with the absorption of $\mathrm{ASR}_{\mathrm{AT}}$ greater than that of $\mathrm{ASR}_{13 \mathrm{C}}$ 
(broken lines in Figure 29a), this suggests that the 13C trans form has a large absorption in the protein pocket of ASR.

We then illuminated the dark-adapted and light-adapted ASR at $480 \mathrm{~nm}$, the isosbestic point of $\mathrm{ASR}_{\mathrm{AT}}$ and $\mathrm{ASR}_{13 \mathrm{C}}$, at $130 \mathrm{~K}$ (Figure 29a). Figure 29b shows time-dependent absorbance changes at each difference absorption maximum (596 and $590 \mathrm{~nm}$ ) of the dark-adapted and light-adapted ASR. The increase of absorbance is greater for the light-adapted ASR, which contains more ASR $_{13 \mathrm{C}}$, and originates also from the larger absorbance of the $\mathrm{K}$ intermediate of $\mathrm{ASR}_{13 \mathrm{C}}$. By considering the molar extinction coefficients of the $\mathrm{K}$ intermediates, the relative quantum yield for the photoisomerization of $\mathrm{ASR}_{13 \mathrm{C}}$ and $\mathrm{ASR}_{\mathrm{AT}}$ was determined to be $0.73( \pm 0.07): 1$. From the data in Figure 26a and $\mathrm{c}$, the branching ratio of $\operatorname{ASR}_{13 \mathrm{C}}$ ( $\mathrm{y}$ in Figure 22) was therefore determined to be $1.06 \pm 0.11$. This value demonstrates that the $\mathrm{K}$ intermediate formed from $\mathrm{ASR}_{13 \mathrm{C}}$ is completely converted into $\mathrm{ASR}_{\mathrm{AT}}$ without regaining the initial state in a photocyclic reaction (Figure 30).

Figure 30. The photoreaction of ASR. Both isomers $\left(\mathrm{ASR}_{\mathrm{AT}}\right.$ and $\left.\mathrm{ASR}_{13 \mathrm{C}}\right)$ convert $100 \%$ yield to another isomer, respectively. This figure is reprinted with permission from Kawanabe et al. [71]. Copyright 2007 American Chemical Society.

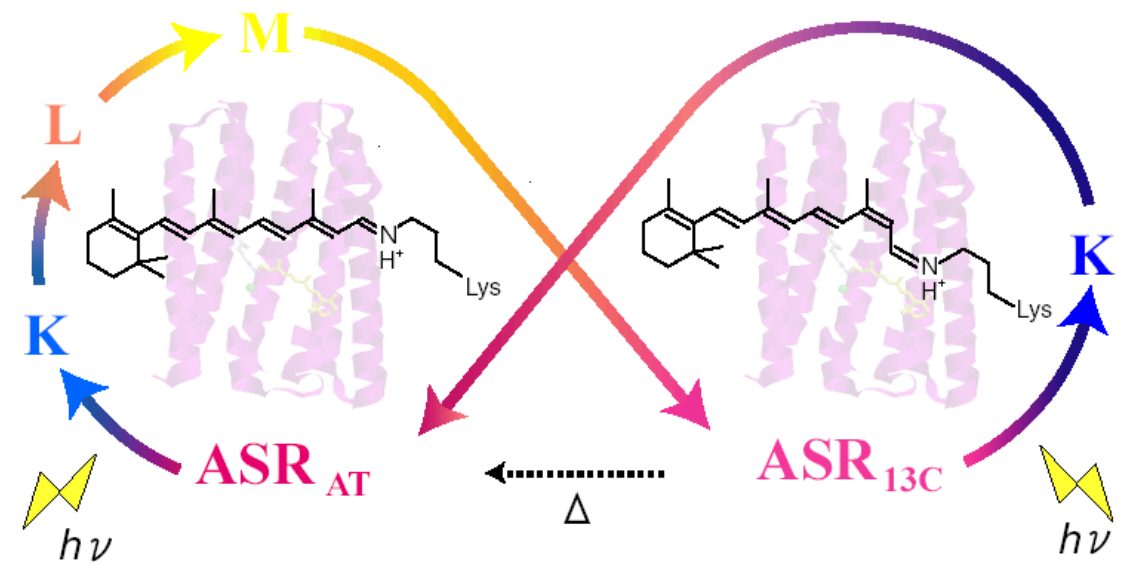

\subsection{Functional Optimization of Photoconversions in Rhodopsins}

The present results reveal that the branching reactions take place with $100 \%$ efficiency, both from $\mathrm{ASR}_{\mathrm{AT}}$ and $\mathrm{ASR}_{13 \mathrm{C}}$. Although the present results were obtained for ASR in liposomes, not in native membranes, this characteristic is highly advantageous for a photochromic sensor. On the other hand, the AT form of BR has $100 \%$ photocyclic efficiency (Figure 27), which is important for the proton pump. Thus, it is concluded that ASR and BR have been optimized for their functions, presumably during evolution. It is intriguing that the structures of the chromophore and its binding pocket are similar between ASR [16] and BR [7], although their amino acid sequences are not highly homologous (60\%). Our FTIR study revealed that hydrogen bond of the Schiff base is similarly strong in ASR and BR, and they are similarly cleaved after retinal photoisomerization [20]. Replacement of aspartate (Asp212 of BR) by proline in ASR (Pro206) is one of the structural differences. Another difference is the hydrogen bonding strength of the water molecule near the Schiff base. BR possesses a strongly hydrogen-bonded water molecule between the Schiff base and its counterion (Asp85), which appears to be a prerequisite 
for proton-pump function [27]. ASR possesses such a water molecule between the Schiff base and its counterion (Asp75) [16], but its hydrogen bond is much weaker [20]. These small differences may be determinants for distinguishing photocyclic or photochromic reactions. Recently, Sudo and Spudich converted BR into a sensory receptor by mutation of three hydrogen-bonding residues [72]. This finding also suggests that distinct functions are determined by small differences. In addition, the $\mathrm{M}$ intermediate is formed during the photoreaction of $\mathrm{ASR}_{\mathrm{AT}}$, like BR, but Asp75 is not protonated [73], presumably because the proton is conducted toward the cytoplasmic domain [74]. Further structural analysis of photoreaction intermediates will provide a better understanding of the mechanism for thermal relaxation of the photoisomerized chromophore.

\section{FTIR Study of the L Intermediate of Anabaena Sensory Rhodopsin: Structural Changes in the Cytoplasmic Region}

The $\mathrm{M}$ intermediate with the deprotonated Schiff base is an important state in proton transport and signal transduction. It has been known that the Schiff base proton is transferred to the counterion (Asp85 in BR) if it is deprotonated. In this case, the proton transfer is toward extracellular side. On the other hand, the previous time-resolved FTIR study of ASR by Shi et al. reported the proton transfer to Asp217 in the cytoplasmic side [74], though Asp75 works as the counterion of the Schiff base in ASR. No proton transfer to Asp75 was also reported by Bergo et al. [73]. This may be reasonable, because another aspartate (Asp212 in BR) is replaced by proline in ASR, and Asp212 plays an important role in the proton transfer in BR [23,75]. On the other hand, Sineshchekov et al. reported that the direction of proton transfer was dependent on the sample conditions, where the direction is toward cytoplasmic and extracellular side for C-terminal truncated and full-length ASR, respectively [76]. According to these results, native full-length ASR in E. coli cells exhibits proton transfer direction the same as in BR.

Thus, the molecular mechanism of ASR activation remains yet unclear. In this study, we applied low-temperature FTIR spectroscopy at $170 \mathrm{~K}$ to the dark-adapted ASR that has predominantly all-trans retinal (97\%) [59]. The obtained $\mathrm{ASR}_{\mathrm{L}}$ minus ASR spectra were similar between the full-length and C-terminally truncated ASR, implying similar protein structural changes for the $\mathrm{L}$ state. The $\mathrm{ASR}_{\mathrm{L}}$ minus ASR spectra were essentially similar to those of BR, but a unique spectral feature was observed in the carboxylic $\mathrm{C}=\mathrm{O}$ stretching region. The bands at $1,722(+)$ and $1,703(-) \mathrm{cm}^{-1}$ were observed at $\mathrm{pH}$, which was reduced at $\mathrm{pH} 7$ and disappeared at $\mathrm{pH}$ 9. The mutation study successfully assigned the bands to the $\mathrm{C}=\mathrm{O}$ stretch of Glu36. Interestingly, Glu36 is located at the cytoplasmic side, and the distance from the retinal Schiff base is about $20 \AA$ (Figure 31). We also observed pH-dependent frequency change of a water stretching vibration, which is located near Glu36. Unique hydrogen-bonding network in the cytoplasmic domain of ASR will be discussed. 
Figure 31. X-ray crystallographic structure of the cytoplasmic region of ASR (PDB entry $1 X I O$ [16]). Top and bottom regions correspond to the cytoplasmic surface and retinal binding pocket, respectively. Green spheres represent water molecules in the cytoplasmic region. Hydrogen-bonds (yellow dashed lines) are inferred from the structure. This figure is reprinted with permission from Kawanabe et al. [77]. Copyright 2008 American Chemical Society.

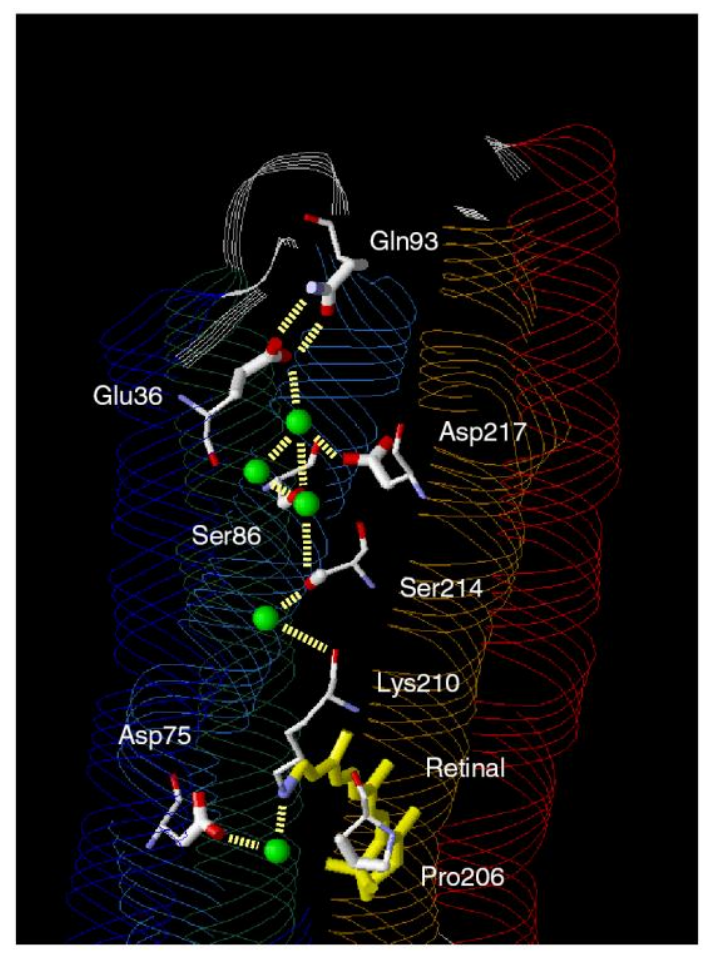

5.1. Comparison of the Difference Infrared Spectra of the L Intermediate of Full-length ASR and Truncated ASR in the 1,800-800 $\mathrm{cm}^{-1}$ Region

The previous photoelectric measurements showed that the direction of charge movement of fulllength ASR was different from that of C-terminally truncated ASR (truncated ASR) for the L and M intermediates, whereas both charge movements were similar for the $\mathrm{K}$ intermediate [76]. This suggests that full-length and truncated ASR have different structural changes in the L and M intermediates. Therefore, we prepared both full-length and truncated ASR, and measured the difference FTIR spectra for the $\mathrm{L}$ intermediate. Figure 32 compares the full-length $\mathrm{ASR}_{\mathrm{L}}$ minus ASR (solid line) and the truncated $\mathrm{ASR}_{\mathrm{L}}$ minus ASR (dotted line) spectra at $170 \mathrm{~K}$ upon hydration with $\mathrm{H}_{2} \mathrm{O}$. As is clearly seen, the spectrum of the full-length ASR is very similar to that of the C-terminally truncated ASR. Thus, the present FTIR spectra for the L intermediate showed no effects of the C-terminal truncation. All data below are shown for the full-length ASR including the mutant proteins. It should be noted that we confirmed similarity of the spectra at $170 \mathrm{~K}$ between full-length and truncated ASR at acidic and alkaline $\mathrm{pH}$ as well, though they could be different at room temperature. 
Figure 32. The full-length (solid line) and truncated (dotted line) $\mathrm{ASR}_{\mathrm{L}}$ minus ASR spectra $(\mathrm{pH} 7)$ in the 1,800-900 $\mathrm{cm}^{-1}$ region. The spectra are measured at $170 \mathrm{~K}$ upon hydration with $\mathrm{H}_{2} \mathrm{O}$. One division of the y-axis corresponds to 0.004 absorbance units. This figure is reprinted with permission from Kawanabe et al [77]. Copyright 2008 American Chemical Society.

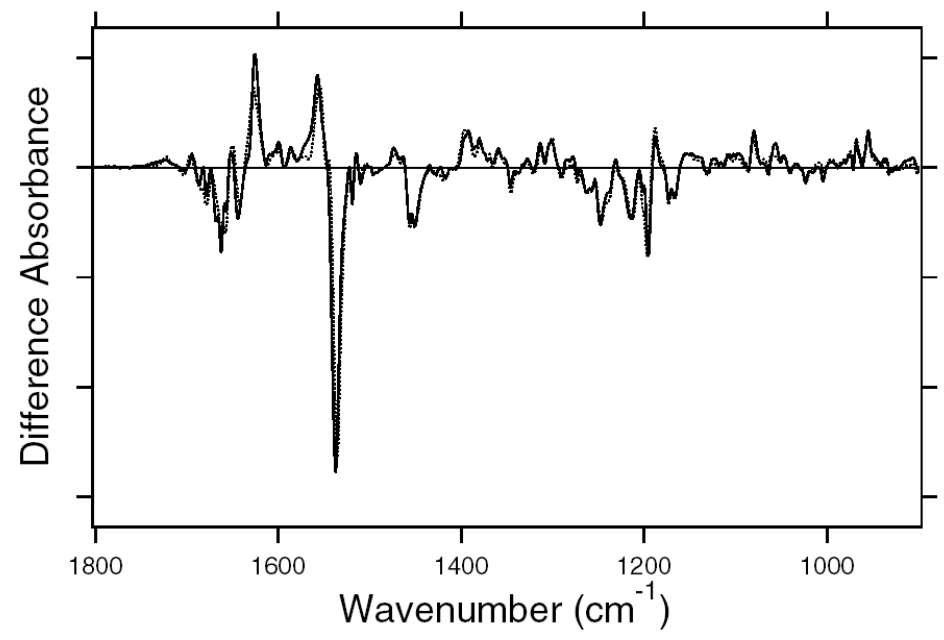

5.2. Comparison of the Difference Infrared Spectra of the L Intermediate of ASR and BR in the 1,800-800 $\mathrm{cm}^{-1}$ Region

Figure 33 compares the $\mathrm{ASR}_{\mathrm{L}}$ minus $\mathrm{ASR}$ (a) and the $\mathrm{BR}_{\mathrm{L}}$ minus $\mathrm{BR}$ spectra (b) at $170 \mathrm{~K}$. The samples were hydrated with $\mathrm{H}_{2} \mathrm{O}$ (solid lines) and $\mathrm{D}_{2} \mathrm{O}$ (dotted lines). In Figure 33a, the negative band at $1,537 \mathrm{~cm}^{-1}$ corresponds to the ethylenic vibration of all-trans retinal in ASR, which exhibits the absorption maximum at $549 \mathrm{~nm}$ [59]. The $\mathrm{ASR}_{\mathrm{K}}$ minus ASR spectrum also showed the negative band at identical frequency [20]. In the case of BR, the ethylenic vibration of the $L$ intermediate is observed at higher frequency $\left(1,550 \mathrm{~cm}^{-1}\right)$ than that of the original state $\left(1,528 \mathrm{~cm}^{-1}\right)$, which corresponds to the blue-shifted absorption maximum of $\mathrm{BR}_{\mathrm{L}}$ [29]. Similarly, illumination of ASR results in the spectral upshift to $1,558 \mathrm{~cm}^{-1}$. Blue-shifted visible absorption of $\mathrm{ASR}_{\mathrm{L}}$ is consistent with our low-temperature UV-visible analysis [71].

C-C stretching vibrations of retinal in the $1,300-1,150 \mathrm{~cm}^{-1}$ region are sensitive to the local structure of the chromophore. Negative bands at 1,255, 1,216, 1,202, and 1,169 $\mathrm{cm}^{-1}$ in Figure $33 \mathrm{~b}$ were assigned to the $\mathrm{C} 12-\mathrm{C} 13, \mathrm{C} 8-\mathrm{C} 9, \mathrm{C} 14-\mathrm{C} 15$, and $\mathrm{C} 10-\mathrm{C} 11$ stretching vibrations of $\mathrm{BR}$, respectively [31]. These bands are typical to all-trans retinal protonated Schiff base but located at higher frequencies corresponding to charge delocalization in the retinal molecule in $\mathrm{BR}$. $\mathrm{BR}_{\mathrm{L}}$ has a 13-cis retinal, resulting in the appearance of a strong positive band at $1,192 \mathrm{~cm}^{-1}$, which is assigned to C10-C11 and C14-C15 stretching vibrations [78]. Essentially similar observation was obtained for ASR. From the similarity in frequency, negative bands at 1,248, 1,215, 1,196, and 1,174 (and/or 1,167) $\mathrm{cm}^{-1}$ can be assigned to C12-C13, C8-C9, C14-C15, and C10-C11 stretching vibrations of ASR (Figure 33a). 
Figure 33. The $\mathrm{ASR}_{\mathrm{L}}$ minus $\mathrm{ASR}$ (a) and the $\mathrm{BR}_{\mathrm{L}}$ minus $\mathrm{BR}$ (b) spectra in the $1,800-900 \mathrm{~cm}^{-1}$ region, which are measured at $\mathrm{pH} 7$ and $170 \mathrm{~K}$ upon hydration with $\mathrm{H}_{2} \mathrm{O}$ (solid line) and $\mathrm{D}_{2} \mathrm{O}$ (dotted line), respectively. One division of the $\mathrm{y}$-axis corresponds to 0.012 absorbance units. This figure is reprinted with permission from Kawanabe et al [77]. Copyright 2008 American Chemical Society.

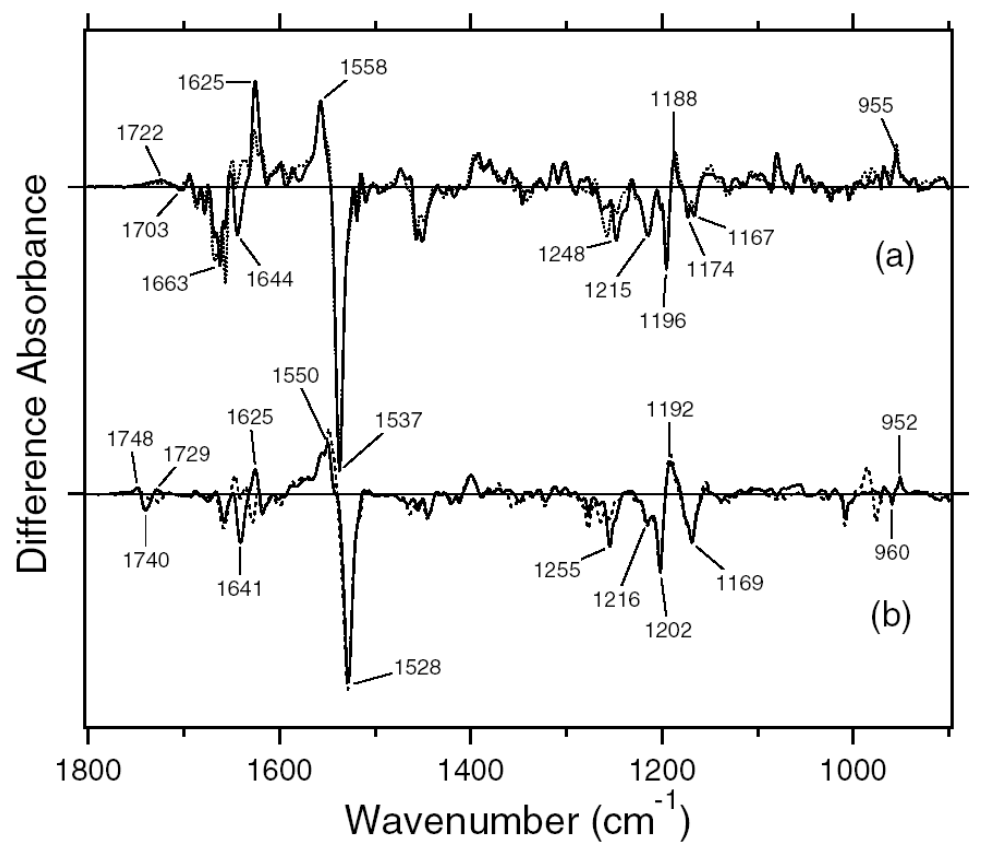

The difference spectra in the $1,110-890 \mathrm{~cm}^{-1}$ region are expanded in Figure 34, where hydrogen-outof-plane (HOOP), N-D in-plane bending and methyl rocking vibrations appear. The presence of strong HOOP modes represents the distortion of the retinal molecule [60]. The $\mathrm{ASR}_{\mathrm{L}}$ minus ASR spectra exhibit two positive peaks at 968 and $955 \mathrm{~cm}^{-1}$, which possibly correspond to the bands at 968 and $951 \mathrm{~cm}^{-1}$ of $\mathrm{BR}_{\mathrm{L}}$, respectively (Figure 34). The bands at $986(+)$ and $976(-) \mathrm{cm}^{-1}$ were assigned to the N-D in-plane bending vibrations of $\mathrm{BR}_{\mathrm{L}}$ and $\mathrm{BR}$, respectively [35]. On the other hand, the $\mathrm{ASR}_{\mathrm{L}}$ minus ASR spectrum does not show clearer $H-D$ exchange dependent bands in this region than the $\mathrm{BR}_{\mathrm{L}}$ minus BR spectrum. The $1,009 \mathrm{~cm}^{-1}$ band in Figure $34 \mathrm{~b}$ is insensitive to the $\mathrm{H}-\mathrm{D}$ exchange and was assigned to the methyl rocking vibration of the retinal in BR. The band at $1,005 \mathrm{~cm}^{-1}$ in Figure $34 \mathrm{a}$ is also assignable to the methyl rocking vibration in ASR. Thus, similar L spectra were observed for ASR and BR.

Amide-I vibrations appear in the $1,700-1,550 \mathrm{~cm}^{-1}$ region together with the $\mathrm{C}=\mathrm{N}$ stretching vibration of the protonated retinal Schiff base (Figure 33). In general, the former is little sensitive to the H-D exchange, whereas the latter exhibits a downshift in $\mathrm{D}_{2} \mathrm{O}$. The bands at $1,641(-)$ and $1,625(+) \mathrm{cm}^{-1}$ were assigned to the $\mathrm{C}=\mathrm{N}$ stretching vibrations of $\mathrm{BR}$ and $\mathrm{BR}_{\mathrm{L}}$, respectively [35]. In the case of ASR, a prominent negative peak at $1,644 \mathrm{~cm}^{-1}$ is assignable to the $\mathrm{C}=\mathrm{N}$ stretch of ASR, because the $\mathrm{D}_{2} \mathrm{O}$-sensitive $1,644 \mathrm{~cm}^{-1}$ band in the $\mathrm{ASR}_{\mathrm{K}}$ minus ASR spectra was identified by use of ${ }^{15} \mathrm{~N}$-lysine labeled ASR [59]. On the other hand, the $\mathrm{C}=\mathrm{N}$ stretch of $\mathrm{ASR}_{\mathrm{L}}$ is not obvious. The positive peak at $1,625 \mathrm{~cm}^{-1}$ is a candidate, whereas the downshifted band was not clearly observed in $\mathrm{D}_{2} \mathrm{O}$ (dotted line in Figure 33a). The H-D independent band at $1,663 \mathrm{~cm}^{-1}$ presumably originates from amide-I vibration. 
The frequency suggests structural changes of a distorted $\alpha$-helix. Since the negative band at $1,663 \mathrm{~cm}^{-1}$ is absent for the $\mathrm{ASR}_{\mathrm{K}}$ minus ASR spectra [20], the structural changes of $\alpha$-helix newly appear in $\mathrm{ASR}_{\mathrm{L}}$.

Figure 34. The $\mathrm{ASR}_{\mathrm{L}}$ minus $\mathrm{ASR}$ (a) and the $\mathrm{BR}_{\mathrm{L}}$ minus $\mathrm{BR}$ (b) spectra in the 1,030-900 $\mathrm{cm}^{-1}$ region, which correspond to hydrogen-out-of-plane (HOOP) vibrations of the retinal chromophore. The sample was hydrated with $\mathrm{H}_{2} \mathrm{O}$ (solid lines) or $\mathrm{D}_{2} \mathrm{O}$ (dotted lines). One division of the $\mathrm{y}$-axis corresponds to 0.0016 absorbance units. This figure is reprinted with permission from Kawanabe et al [77]. Copyright 2008 American Chemical Society.

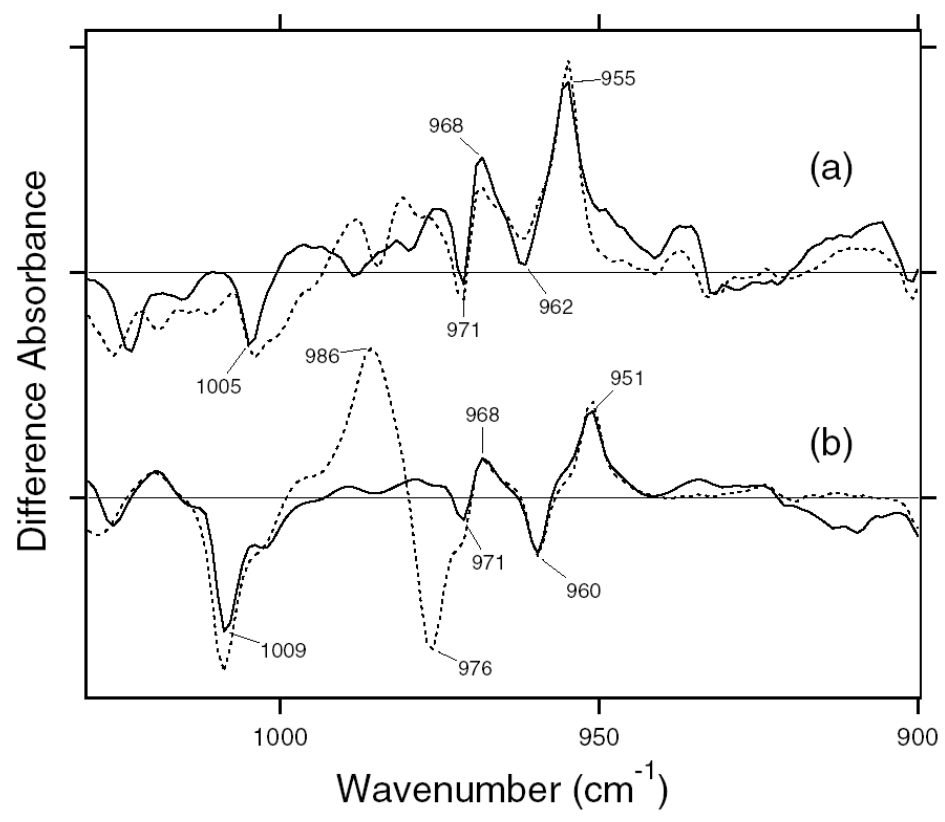

5.3. Comparison of the Difference Infrared Spectra of the L Intermediate in Protonated Carboxylic Acid (1,800-1,700 $\left.\mathrm{cm}^{-1}\right)$ Region

The infrared difference spectra in this frequency region mainly monitor the structural changes of protonated carboxylic acids. In the $\mathrm{BR}_{\mathrm{L}}$ minus $\mathrm{BR}$ difference spectra, the bands at $1,748(+)$ and $1,729(+) \mathrm{cm}^{-1}$ were assigned to the $\mathrm{C}=\mathrm{O}$ stretching vibrations of the protonated Asp96 and Asp115, respectively, while large negative band at $1,740 \mathrm{~cm}^{-1}$ contains the corresponding bands of Asp96 and Asp115 in the unphotolyzed state (Figure 33b) [42]. The corresponding amino acids in ASR are Ser86 and Asn105, so that we did not expect any peaks in this frequency region. Nevertheless, Figure 33a shows a broad positive peak at $1,722 \mathrm{~cm}^{-1}$ as well as a negative feature at $1,703 \mathrm{~cm}^{-1}$, suggesting structural perturbation of carboxylic acids upon formation of $\mathrm{ASR}_{\mathrm{L}}$. It should be noted that the bands do not originate from the contribution of $\mathrm{ASR}_{\mathrm{M}}$, because UV-visible spectroscopy confirmed no formation of $\mathrm{ASR}_{\mathrm{M}}$ at $170 \mathrm{~K}$ [71]. The absence of a clear negative band at around $1,400 \mathrm{~cm}^{-1}$, characteristic of $\mathrm{COO}^{-}$stretching frequency of negatively charged carboxylates, suggests that appearance of the carboxylic $\mathrm{C}=\mathrm{O}$ stretch at $1,722 \mathrm{~cm}^{-1}$ in $\mathrm{ASR}_{\mathrm{L}}$ is not due to the newly protonated species, but rather due to the frequency shift from $1,703 \mathrm{~cm}^{-1}$ in ASR (Figure 33a). 
To further examine the spectral feature in this region, we measured $\mathrm{ASR}_{\mathrm{L}}$ minus ASR spectra at acidic ( $\mathrm{pH}$ 5) and alkaline $(\mathrm{pH} 9) \mathrm{pH}$ in addition to $\mathrm{pH} 7$ (Figure 33a). We also measured the $\mathrm{ASR}_{\mathrm{L}}$ minus ASR spectra of D217N and E36Q mutant proteins to identify the responsible carboxylic acid. Figure 35 clearly shows that $\mathrm{ASR}_{\mathrm{L}}$ is formed at different $\mathrm{pH}$ values $(5,7$, and 9 in a, b, and c, respectively), as well as for the D217N (d) and E36Q (e) mutants. Figure 36 highlights the carboxylic $\mathrm{C}=\mathrm{O}$ stretching region, where all spectra were normalized by use of the negative $1,196 \mathrm{~cm}^{-1}$ band (Figure 35). Although the positive peak at $1,722 \mathrm{~cm}^{-1}$ was broad at pH 7 (Figure 36b), it was enhanced at $\mathrm{pH} 5$ (Figure 36a). In contrast, the $1,722 \mathrm{~cm}^{-1}$ band completely disappeared at $\mathrm{pH} 9$ (Figure 36c). Spectral downshift to $1,717 \mathrm{~cm}^{-1}$ in $\mathrm{D}_{2} \mathrm{O}$ (Figure $36 \mathrm{a}$ ) is typical for carboxylic $\mathrm{C}=\mathrm{O}$ stretching vibrations. Thus, we identify the positive band at $1,722 \mathrm{~cm}^{-1}$ in the $\mathrm{ASR}_{\mathrm{L}}$ minus ASR spectra as a carboxylic $\mathrm{C}=\mathrm{O}$ stretch, whose pKa was estimated between 6 and 7 .

Figure 35. The $\mathrm{ASR}_{\mathrm{L}}$ minus ASR infrared spectra of the wild type at $\mathrm{pH} 5$ (a), pH 7 (b), and $\mathrm{pH} 9$ (c), D217N at pH 5 (d) and E36Q at pH 5 (e) in the 1,800-900 $\mathrm{cm}^{-1}$ region. The spectra are measured at $170 \mathrm{~K}$ upon hydration with $\mathrm{H}_{2} \mathrm{O}$. One division of the $\mathrm{y}$-axis corresponds to 0.009 absorbance units. This figure is reprinted with permission from Kawanabe et al [77]. Copyright 2008 American Chemical Society.

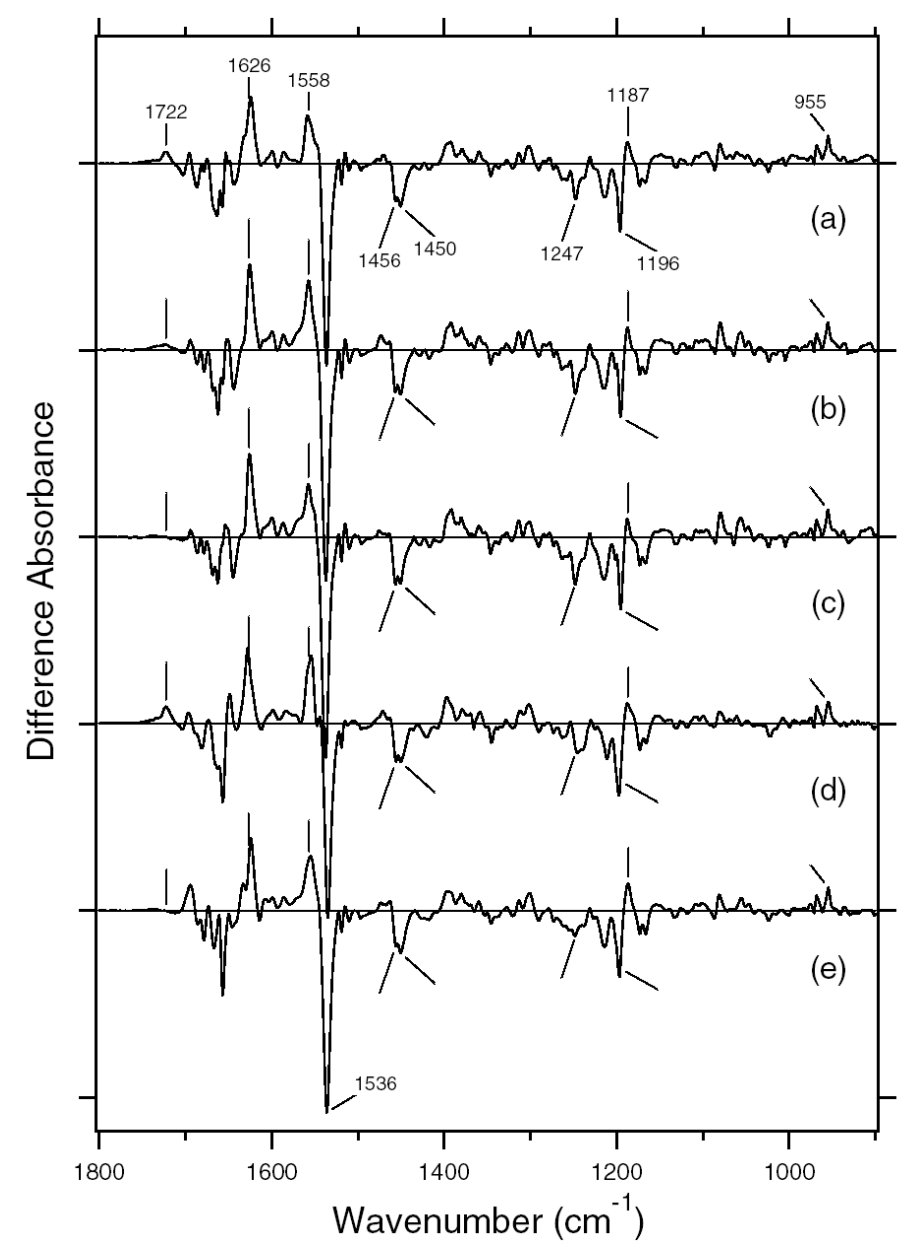

The negative band at $1,703 \mathrm{~cm}^{-1}$ exhibits similar $\mathrm{pH}$ dependence to that of the $1,722 \mathrm{~cm}^{-1}$ band, being enhanced at $\mathrm{pH} 5$, but disappearing at $\mathrm{pH} 9$ (Figure 36a-c). In addition, the $1,703 \mathrm{~cm}^{-1}$ band is downshifted in $\mathrm{D}_{2} \mathrm{O}$ (Figure 36a), though the shifted negative band was not clearly observable because 
of the strong peaks at $1,695(+) / 1,687(-) \mathrm{cm}^{-1}$ (Figure 33a). Similar $\mathrm{pH}$ dependence strongly suggests that the bands at $1,722(+) / 1,703(-) \mathrm{cm}^{-1}$ originate from the same carboxylic group. The absence of $\mathrm{pH}$ dependent bands at around $1,400 \mathrm{~cm}^{-1}$ (Figure 35) also supports this interpretation.

Finally, the remaining question is the location of the carboxylic group responsible for this spectral feature. The previous time-resolved FTIR study observed a positive carboxylic $\mathrm{C}=\mathrm{O}$ stretch at $1,716 \mathrm{~cm}^{-1}$ in the $\mathrm{ASR}_{\mathrm{M}}$ minus ASR spectra, and assigned the band to Asp217 located at the cytoplasmic region, because the band disappeared for D217N, but not for E36Q [74]. Interestingly, the $1,716 \mathrm{~cm}^{-1}$ band in $\mathrm{ASR}_{\mathrm{M}}$ was also $\mathrm{pH}$-dependent, whose $\mathrm{pKa}$ was between 6 and 7, but the $\mathrm{pH}$ dependence was opposite to the present case. Namely, the positive band at $1,716 \mathrm{~cm}^{-1}$ was observed at alkaline $\mathrm{pH}$, but not at acidic $\mathrm{pH}$, and the authors interpreted that Asp217 is protonated at acidic $\mathrm{pH}$ in the unphotolyzed state [74].

They did not observe frequency change of Asp217 at acidic $\mathrm{pH}$, suggesting no structural changes of Asp217 at acidic conditions. Thus, there has been no information about the $\mathrm{C}=\mathrm{O}$ stretching frequency of Asp217 at acidic $\mathrm{pH}$, and Asp217 is a possible candidate for the bands at 1,722(+)/1,703(-) $\mathrm{cm}^{-1}$ in the $\mathrm{ASR}_{\mathrm{L}}$ minus ASR spectra. Here we also measured the spectra of the E36Q mutant, as Glu36 is located near Asp217 (Figure 31). Figures 36d and 36e show the carboxylic $\mathrm{C}=\mathrm{O}$ stretching region in the $\mathrm{ASR}_{\mathrm{L}}$ minus ASR spectra of D217N and E36Q, respectively. Similar difference spectra in other frequency regions ensure the normal formation of $\mathrm{ASR}_{\mathrm{L}}$ for these mutants (Figure 35).

Figure 36. The $\mathrm{ASR}_{\mathrm{L}}$ minus ASR infrared spectra of the wild type at $\mathrm{pH} 5$ (a), pH 7 (b), and $\mathrm{pH} 9$ (c), D217N at pH 5 (d) and E36Q at $\mathrm{pH} 5$ (e) in the 1,740-1,700 $\mathrm{cm}^{-1}$ region. The sample was hydrated with $\mathrm{H}_{2} \mathrm{O}$ (solid lines) or $\mathrm{D}_{2} \mathrm{O}$ (dotted lines). One division of the $\mathrm{y}$-axis corresponds to 0.0008 absorbance units. This figure is reprinted with permission from Kawanabe et al [77]. Copyright 2008 American Chemical Society.

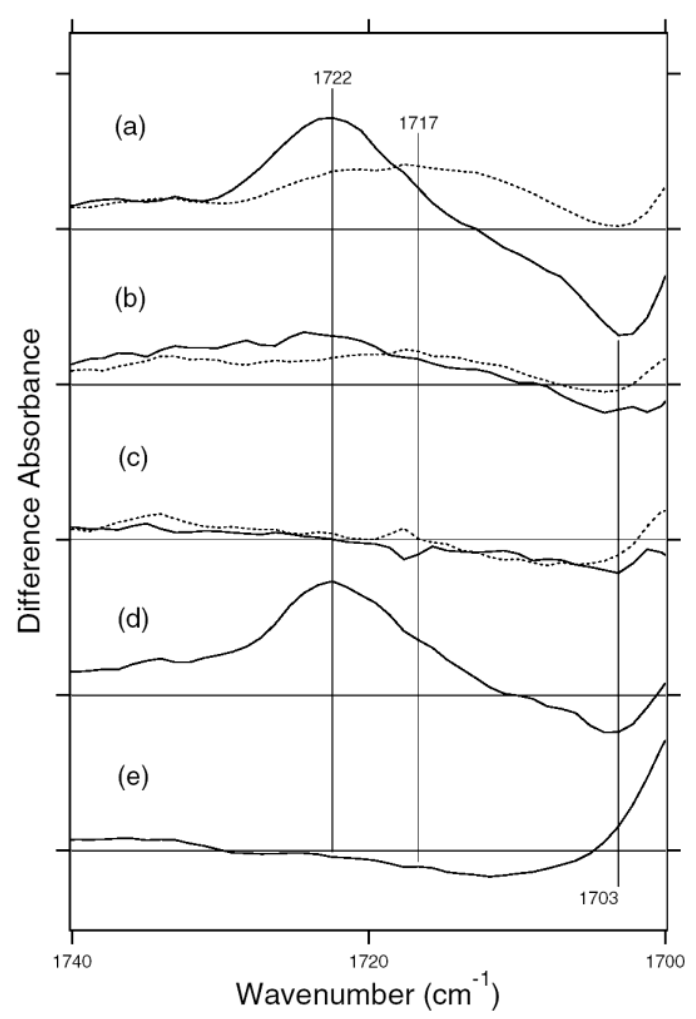


The bands at $1,722(+) / 1,703(-) \mathrm{cm}^{-1}$ were reproduced in Figure 36d, indicating that they do not originate from Asp217. On the other hand, the bands at 1,722(+)/1,703(-) $\mathrm{cm}^{-1}$ completely disappeared for E36Q (Figure 36e). Thus, we assigned the bands to Glu36. It is generally accepted that the $\mathrm{C}=\mathrm{O}$ stretching vibrations appear at lower frequency for Glu than for Asp, but the frequency of Glu36 at $1,703 \mathrm{~cm}^{-1}$ in ASR is particularly unusual. The frequency is very low, indicating that Glu36 forms a strong hydrogen bond in the unphotolyzed state, which is weakened by structural changes upon formation of $\mathrm{ASR}_{\mathrm{L}}$ as shown by the upshift to $1,722 \mathrm{~cm}^{-1}$.

\subsection{Comparison of the Difference Infrared Spectra of the L Intermediate in Water O-D Stretching} Frequency $\left(2,750-2,500 \mathrm{~cm}^{-1}\right)$ Region

The $\mathrm{ASR}_{\mathrm{L}}$ minus ASR spectra clearly show hydrogen-bonding alteration of Glu36. Since there is a water cluster in the cytoplasmic region near Glu36 (Figure 31), detecting water signals in the $\mathrm{ASR}_{\mathrm{L}}$ minus ASR spectrum is important. It was not easy, because $\mathrm{ASR}_{\mathrm{L}}$ decays to the 13-cis form, not to the original all-trans form, and the sample was dark-adapted again before the next measurement. Nevertheless, we successfully measured the spectra in the frequency region of water $\mathrm{O}-\mathrm{D}$ stretching vibrations in $\mathrm{D}_{2} \mathrm{O}$. Figures $37 \mathrm{a}, 37 \mathrm{~b}$, and $37 \mathrm{c}$ show the $\mathrm{ASR}_{\mathrm{L}}$ minus ASR spectra of the wild type at pH 5, 7, and 9, respectively.

Figure 37. The $\mathrm{ASR}_{\mathrm{L}}$ minus ASR infrared spectra of the wild type at pH 5 (a), pH 7 (b), and $\mathrm{pH} 9$ (c), E36Q at pH 5 (d) and pH 9 (e) in the 2,750-2,500 $\mathrm{cm}^{-1}$ region on red spectra. The spectra are measured at $170 \mathrm{~K}$ upon hydration with $\mathrm{D}_{2} \mathrm{O}$. Blue spectrum is hydrated with $\mathrm{D}_{2}{ }^{18} \mathrm{O}$ at $\mathrm{pH} 7$ and red dotted lines are corresponding to WT spectra at each $\mathrm{pH}$. One division of the $\mathrm{y}$-axis corresponds to 0.0009 absorbance units. This figure is reprinted with permission from Kawanabe et al. [77]. Copyright 2008 American Chemical Society.

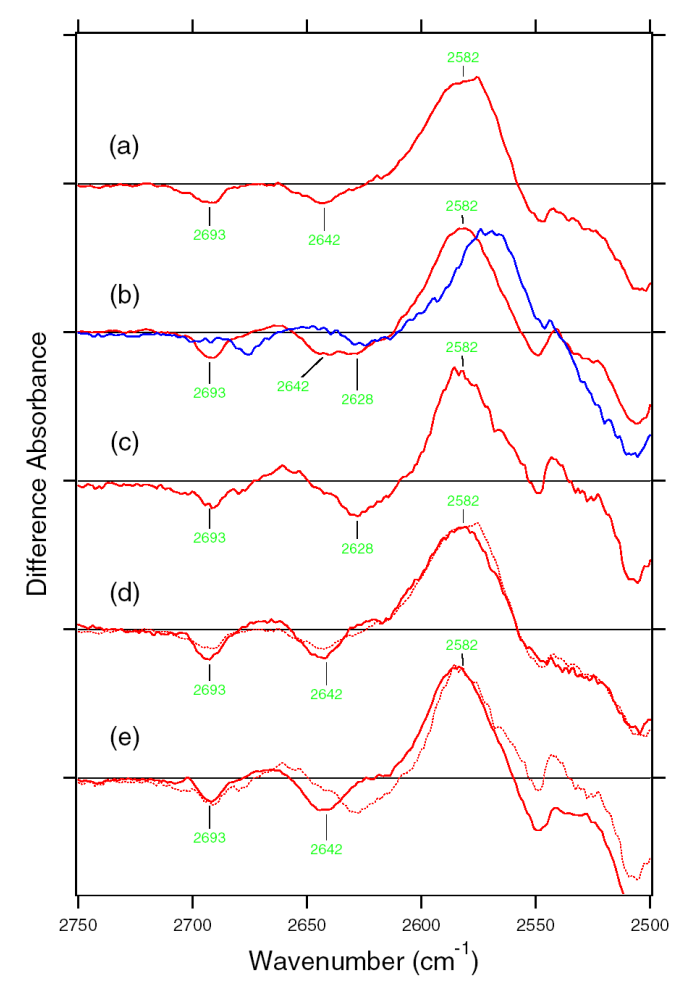


The spectra show a negative peak at $2,693 \mathrm{~cm}^{-1}$ and a positive peak at $2,582 \mathrm{~cm}^{-1}$ at all $\mathrm{pH}$. Since the peaks are downshifted upon hydration with $\mathrm{D}_{2}{ }^{18} \mathrm{O}$, they originate from $\mathrm{O}-\mathrm{D}$ stretching vibrations of water. The negative peaks at 2,642 and $2,628 \mathrm{~cm}^{-1}$ in Figure $37 \mathrm{~b}$ similarly show the isotope shift of water, indicating that they originate from water O-D stretches. Interestingly, a single peak only exists at 2,642 and 2,628 $\mathrm{cm}^{-1}$ for the spectra at $\mathrm{pH} 5$ (Figure 37a) and $\mathrm{pH} 9$ (Figure 37c), respectively. This observation implies that the frequency of the water O-D stretch is $\mathrm{pH}$-dependent, being downshifted at higher $\mathrm{pH}$. The $\mathrm{pKa}$ is located at about 7, which is coincident with that of Glu36 (Figure 36). Similar pKa of the water O-D stretch at $2,650-2,620 \mathrm{~cm}^{-1}$ to that of Glu36 suggests that the water molecule is located near Glu36. This is indeed the case, because the $\mathrm{pH}$ dependence of the water O-D stretch is abolished in E36Q. Figure 37d shows identical spectra between the wild type (dotted line) and E36Q (solid line) at $\mathrm{pH} 5$. However, the frequency shift of the negative band from $2,642 \mathrm{~cm}^{-1}$ to $2,628 \mathrm{~cm}^{-1}$ in the wild type at pH 9 was absent in E36Q (solid line in Figure 37e). This observation suggests that deprotonation of Glu36 at $\mathrm{pH}>7$ is correlated with the frequency shift of the water O-D stretch from $2,642 \mathrm{~cm}^{-1}$ to $2,628 \mathrm{~cm}^{-1}$. The straightforward interpretation is that the water directly interacts with Glu36. It should be noted that the water O-D stretch at 2,650-2,620 $\mathrm{cm}^{-1}$ represents a weak hydrogen bond, and we presumably monitor the free O-D stretch of a water molecule interacting with Glu36.

\subsection{The Structure of $A S R_{L}$}

In this study, we report the $\mathrm{ASR}_{\mathrm{L}}$ minus ASR spectra measured by low-temperature FTIR spectroscopy. Although photoinduced current measurements of $\mathrm{ASR}_{\mathrm{L}}$ and $\mathrm{ASR}_{\mathrm{M}}$ reported the different direction of charge signal between C-terminally truncated and full-length ASR [76], the present FTIR study showed almost identical $\mathrm{ASR}_{\mathrm{L}}$ and ASR spectra for them (Figure 32). While the spectral features were essentially similar to those for $\mathrm{BR}$, a unique feature was obtained for the carboxylic $\mathrm{C}=\mathrm{O}$ stretching frequency region for ASR. The $\mathrm{pH}$-dependent bands were observed at $1,722(+) / 1,703(-) \mathrm{cm}^{-1}$ in the $\mathrm{ASR}_{\mathrm{L}}$ minus ASR spectra, which were assigned to Glu36. $\mathrm{pH}$-dependent water $\mathrm{O}-\mathrm{D}$ stretching vibrations in $\mathrm{D}_{2} \mathrm{O}$ were also observed at 2,642 and 2,628 $\mathrm{cm}^{-1}$ for the unphotolyzed state of ASR at pH 5 and $\mathrm{pH}$ 9, respectively. These bands of pKa were estimated to be between 6 and 7. According to the X-ray structure of ASR, Glu36 is located near the cytoplasmic surface (Figure 31), and the distances from the Schiff base nitrogen of the retinal chromophore to the side-chain oxygens of Glu36 are 19.3 and $20.2 \AA$ [16]. The present study clearly shows that formation of $\mathrm{ASR}_{\mathrm{L}}$ accompanies hydrogen-bonding alteration of Glu36. Since $\mathrm{ASR}_{\mathrm{L}}$ is formed at $170 \mathrm{~K}$, this fact demonstrates structural alteration propagating over $20 \AA$ at such low temperatures.

Spectral feature of the water signal in the $\mathrm{ASR}_{\mathrm{L}}$ minus $\mathrm{ASR}$ spectrum resembles that in the $\mathrm{BR}_{\mathrm{L}}$ minus $\mathrm{BR}$ spectrum at 2,700-2,500 $\mathrm{cm}^{-1}$ [26]. In particular, an intense positive broadband at 2,630-2,550 $\mathrm{cm}^{-1}\left(\mathrm{O}-\mathrm{H}\right.$ stretch at 3,550-3,450 $\left.\mathrm{cm}^{-1}\right)$ has been regarded as a characteristic for the $\mathrm{L}$ state of BR [26]. However, our recent time-resolved FTIR spectroscopy clearly showed the absence of the band for $\mathrm{BR}_{\mathrm{L}}$ at room temperature, and we concluded that such water signal is a low-temperature artifact, or a feature peculiar at low temperature $(170 \mathrm{~K})$ where $\mathrm{L}$ is stable [79]. This may be also true for ASR. However, it should be noted that the cryotrapped L state is considerably relaxed to the original state in BR [80,81], but decays to the subsequent intermediates in ASR by warming [71]. This 
suggests different protein dynamics between ASR and BR, but room-temperature FTIR study of ASR is required for further understanding.

\subsection{Hydrogen-Bonding Structures in the Cytoplasmic Domain of ASR and ASR $R_{L}$}

The present study showed that the pKa of Glu36 is between 6 and 7 in ASR, and its hydrogen bonding is significantly altered upon formation of $\mathrm{ASR}_{\mathrm{L}}$. Previous FTIR study reported the pKa of Asp217 being also between 6 and 7 in ASR [74]. According to these observations, Glu36 and Asp217 are both protonated at low $\mathrm{pH}$, while being both deprotonated at high $\mathrm{pH}$. However, the latter may be unlikely, because Glu36 and Asp217 are located close to each other (Figure 31). Here we propose a possible model for activation of ASR based on these observations and X-ray structure [16], shown in Figure 38. It should be noted that the three water molecules constitute a cluster structure in the region of Glu36 and Asp217 (Figure 31), which has to be taken into the account.

Figure 38. Possible models of hydrogen-bonding alterations in the cytoplasmic region of ASR (see text for details).

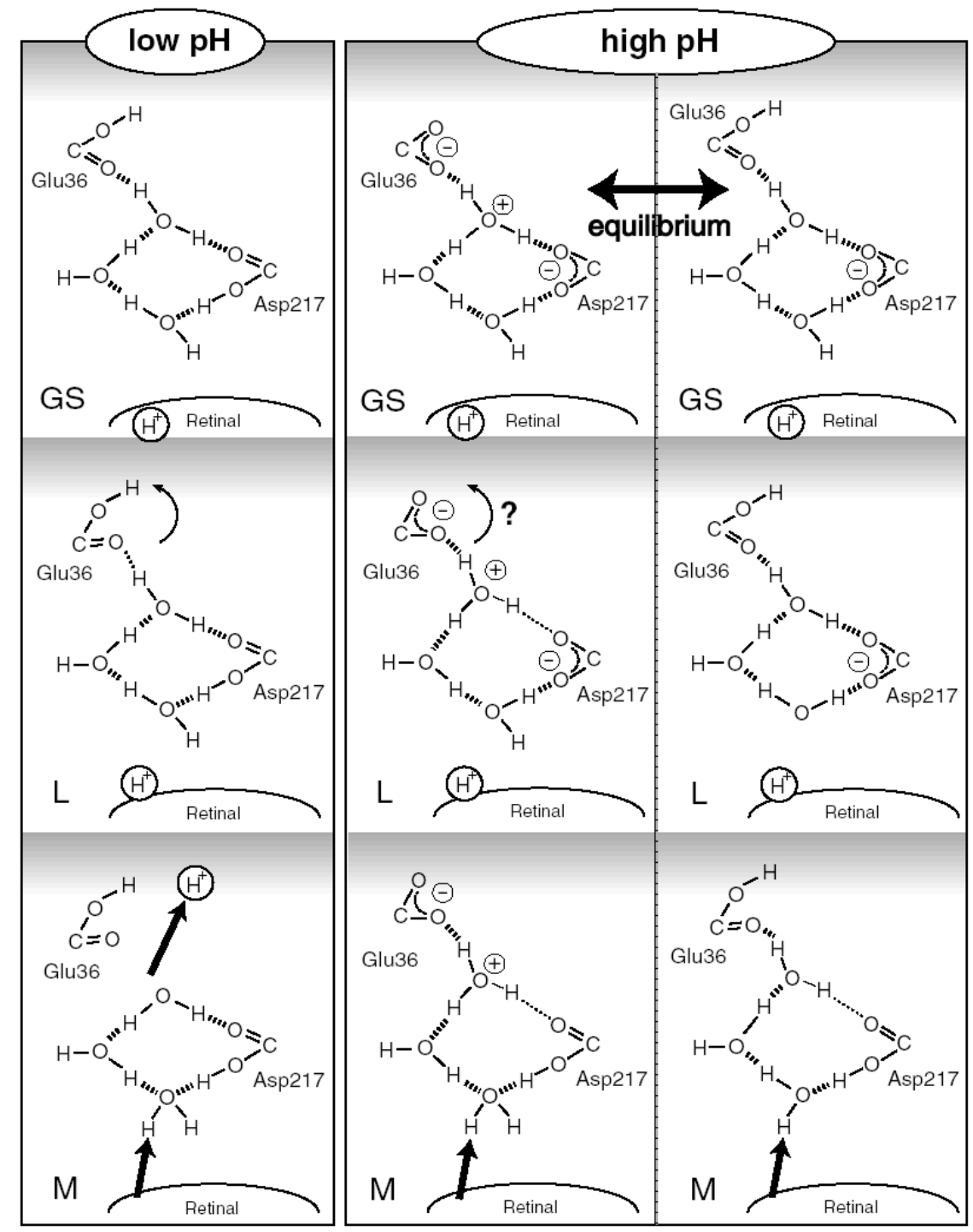


At low pH, Glu36 and Asp217 are both protonated in the unphotolyzed state. Hydrogen bond of Glu36 is remarkably strong $\left(\mathrm{C}=\mathrm{O}\right.$ stretch at $\left.1,703 \mathrm{~cm}^{-1}\right)$, where internal water molecules must play important roles (Figure 38, left panel). Upon $\mathrm{ASR}_{\mathrm{L}}$ formation, the hydrogen bond of Glu36 is weakened $\left(\mathrm{C}=\mathrm{O}\right.$ stretch at $\left.1,722 \mathrm{~cm}^{-1}\right)$. The absence of the bands for Asp217 in the ASR $\mathrm{L}_{\mathrm{L}}$ minus ASR spectra (Figure 36) implies that no hydrogen-bonding alteration of this residue occurs. Therefore, we propose that the interaction between Glu36 and the water cluster is weakened, while that between Asp217 and the water cluster is unchanged (Figure 38, left panel). Then, the L-M transition accompanies proton release to the cytoplasmic aqueous phase through the Glu36-Asp217 region.

At high $\mathrm{pH}$, we first postulate that Asp217 is deprotonated according to the previous results [74]. Then, there are two possibilities on the protonation state of Glu36, either deprotonated (Figure 38, middle panel) or protonated (Figure 38, right panel). Figure 38, middle panel shows both Glu36 and Asp217 deprotonated, and the positively charged water cluster $\left(\mathrm{H}_{3} \mathrm{O}^{+}\right.$or $\left.\mathrm{H}_{7} \mathrm{O}_{3}{ }^{+}\right)$stabilizes the two negative charges. The structure may resemble the proton release group of BR, where water cluster stabilizes Glu194 and Glu204. Experimental evidence of protonated water cluster was first reported by the group of Dr. Gerwert as a continuum band at 2,200-1,800 $\mathrm{cm}^{-1}$ in the room-temperature $\mathrm{BR}_{\mathrm{M}}$ minus BR spectra [82,83], and we recently identified that the continuum band contains water signal [80]. $\mathrm{ASR}_{\mathrm{L}}$ formation does not change the hydrogen-bonding network at the cytoplasmic side, because there is no $\mathrm{pH}$-dependent signal at around $1,400 \mathrm{~cm}^{-1}$ (Figure 35), but hydrogen-bonding interaction may be altered between Glu36 and the water molecules. According to this model, the L-M transition accompanies proton transfer from the Schiff base to Asp217.

Another model at high $\mathrm{pH}$ is based on Glu36 being protonated (Figure 38, right panel). In this case, the $\mathrm{pH}$-dependence can be interpreted not by the direct titration of Glu36, but of other parts of the protein. In addition, hydrogen bond of protonated Glu36 is not changed between ASR and ASR $_{\mathrm{L}}$, so that there are no bands in the $1,740-1,700 \mathrm{~cm}^{-1}$ region (Figure $36 \mathrm{c}$ ). $\mathrm{ASR}_{\mathrm{L}}$ formation does not change the hydrogen bond of Asp217, because there is no $\mathrm{pH}$-dependent signal at around $1,400 \mathrm{~cm}^{-1}$ (Figure 35). The L-M transition accompanies proton transfer from the Schiff base to Asp217. At this moment, we cannot exclude either of the two models at high $\mathrm{pH}$. The latter (Figure 38, right panel) may be less plausible, because it predicts no hydrogen-bonding alterations in the cytoplasmic region between ASR and $\mathrm{ASR}_{\mathrm{L}}$. On the other hand, the two model structures may be in equilibrium. In this regard, detecting water signals in the $\mathrm{ASR}_{\mathrm{L}}$ minus ASR and $\mathrm{ASR}_{\mathrm{M}}$ minus ASR spectra is very important, though it is not easy because of the photochromic nature of ASR [71]. The spectral analysis of water is our future focus.

\subsection{Characteristic Features of Photoreaction in ASR}

By use of low-temperature UV-visible spectroscopy, we recently revealed that the stable photoproduct of the all-trans form is $100 \%$ 13-cis, and that of the 13-cis form is $100 \%$ all-trans [71]. This was entirely unique for archaeal-type rhodopsins, because functionally important states known so far were only derived from the all-trans form, and the photocycle of the all-trans form without branching into the 13-cis stable states has been the common mechanism. The complete photocycling for the proton pump in BR and the complete photochromism for the chromatic sensor of ASR are highly advantageous for their functions. Although the protein structures are similar between ASR and BR [16], 
the present study suggests that the migration of protons to the cytoplasmic side is correlated with the unique photoreactions of ASR.

ASR has Asp75 as a counterion of the retinal chromophore, which corresponds to Asp85 in BR. Nevertheless, the Schiff base proton is transferred not to Asp75 [73,74], but to Asp217 in the cytoplasmic region. What is the mechanism of proton transfer in the opposite direction? The present FTIR spectroscopy of the L intermediate revealed similar structural changes for the chromophores of ASR and BR, suggesting the importance of the surrounding protein moiety. It should be noted that Asp212 in BR is replaced by proline (Pro206) in ASR. Previous studies reported the important role of Asp212 during the $\mathrm{M}$ formation, and we proposed a hydration switch mechanism as the primary cause of proton transfer reaction in BR. In this mechanism, the bridged water molecule between the Schiff base and Asp85 forms a strong hydrogen bond transiently, which leads to the proton transfer to Asp85 [20]. Lack of aspartate at position 206 would be significant for ASR. In this regard, we found the absence of strongly hydrogen-bonded water molecules in ASR [20]. Since there is a positive correlation between the strongly hydrogen-bonded water molecules and the proton pumping activity, weakly hydrogen-bonded water molecules in the Schiff base region may be the key element. Interestingly, the replacement of Pro206 to Asp was not sufficient for ASR to function as a BR-like proton pump [84]. Since the Schiff base proton is transferred to the cytoplasmic side, ASR is a very good model system to study the general mechanism of proton pumps in archaeal-type rhodopsins.

Figure 39. The structural changes of ASR in L-intermediate. (Left) The $\mathrm{ASR}_{\mathrm{L}}$ minus ASR infrared spectra in protonated carboxylic acid region. (Right) The structure of ASR in cytoplasmic surface. Yellow broken lines indicates hydrogen bond, green spheres are water molecules. This figure is reprinted with permission from TOC of Kawanabe et al [77]. Copyright 2008 American Chemical Society.

The structural changes at the cytoplasmic surface in $\mathrm{ASR}_{\mathrm{L}}$
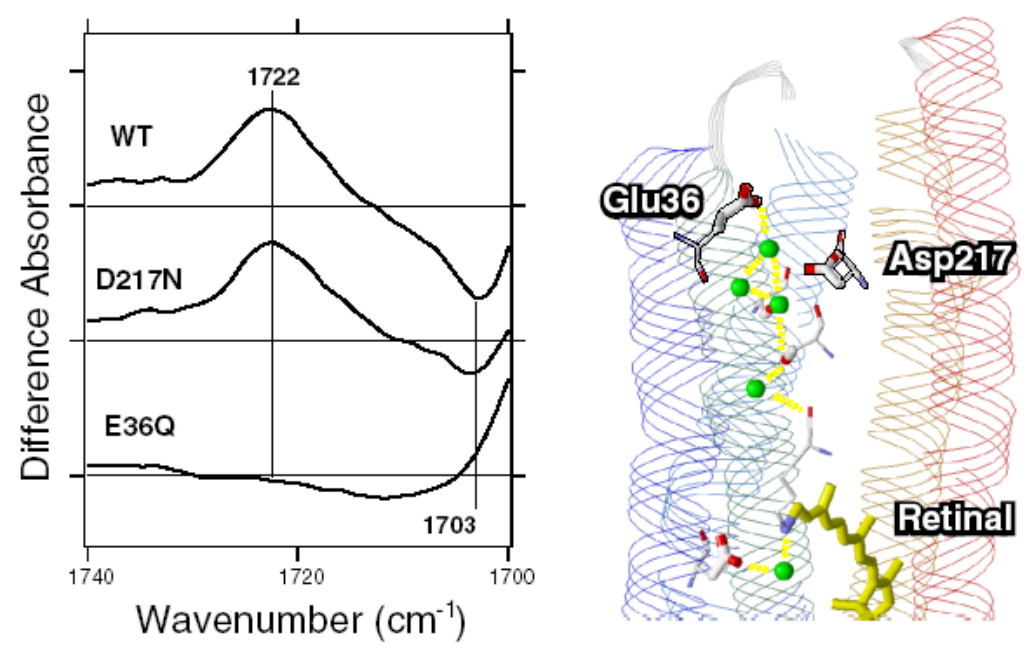

\section{Experimental Section}

ASR Sample Preparation. In the present study, we prepared $C$-terminally truncated and full-length ASR according to the method described previously [15,71,84]. The E36Q and D217N mutants were 
designed based on the full-length ASR, which were produced by a two-step megaprimer PCR method [85], with two oligonucleotides (COSMO, Seoul, Korea): E36Q F-50-CAG TAC CAA TAC CTT GTG GCG ATG- 30 and D217N R-50-GTA AAT TCA GAA AAA CTA AAT C-30. The final PCR products were cloned into plasmid pKJ606 [86], derived from pMS107, by replacing the original insert with XbaI/NotI digestion. After ligation the plasmids were transformed in E. coli strain DH5 $\alpha$. All of the mutations were confirmed by DNA sequencing (COSMO, Seoul, Korea). E. coli strain BL21 (Stratagene) was transformed by introducing pMS107-derivative plasmid [15], which encodes the wildtype, E36Q and D217N opsin, and was grown in 2xYT medium in the presence of ampicillin $(50 \mu \mathrm{g} / \mathrm{ml})$ at $38{ }^{\circ} \mathrm{C}$. Three hours after IPTG induction with addition of $10 \mu \mathrm{M}$ all-trans retinal, pink-colored cells were harvested, sonicated, solubilized by $1 \% \mathrm{DM}$, and purified by a $\mathrm{Ni}^{2+}-\mathrm{NTA}$ column. The purified ASR was then reconstituted into PC liposomes by removing the detergent with Bio-Beads, where the molar ratio of the added PC to ASR was 30:1. The liposomes were washed three times with a buffer [2 $\mathrm{mM}$ sodium phosphate ( $\mathrm{pH}$ 7.0)]. A $40 \mu \mathrm{L}$ aliquot was deposited on a $\mathrm{BaF}_{2}$ window of $18 \mathrm{~mm}$ diameter and dried in a glass vessel that was evacuated by an aspirator. $\left[\zeta^{-15} \mathrm{~N}\right]$ Lysine labeled ASR was prepared as was done for $\left[\zeta_{-}{ }^{15} \mathrm{~N}\right]$ lysine labeled pharaonis phoborhodopsin [87].

\subsection{FTIR Spectroscopy}

FTIR spectroscopy was performed as described previously [36]. Since the all-trans form is most abundant for the dark-adapted ASR [15], ASR films were kept in the dark for 3 days. Completely dark-adapted ASR was hydrated with $\mathrm{H}_{2} \mathrm{O}, \mathrm{D}_{2} \mathrm{O}$, or $\mathrm{D}_{2}{ }^{18} \mathrm{O}$ before measurements. Then, the sample was placed in a cryostat (DN-1,704, Oxford) mounted with the cell for the FTIR spectrometer (FTS-40, Bio-Rad). The cryostat was equipped with a temperature controller (ITC-4, Oxford), and the temperature was regulated with $0.1 \mathrm{~K}$ precision. All the experimental procedures were performed in the dark or under dim red light $(>670 \mathrm{~nm}$ ) before the spectroscopic measurement.

\subsection{Accumulation of $A T-A S R_{K}$.}

Photoreactions of the all-trans and 13-cis forms strongly depend on the illumination wavelength in ASR. It is required that we reduce the extent of the photoreaction of the 13-cis form as much as possible. By using a marker band in the fingerprint $\left(1,200-1,100 \mathrm{~cm}^{-1}\right)$ region, we established the following illumination conditions at $77 \mathrm{~K}$, where difference spectra depicted the photoreaction of the 13 -cis form at $<20 \%$. Illumination with $543 \mathrm{~nm}$ light at $77 \mathrm{~K}$ for 1 min converted ASR to ASR $_{\mathrm{K}}$. $\mathrm{ASR}_{\mathrm{K}}$ was reconverted to ASR upon illumination with $>590 \mathrm{~nm}$ light for $1 \mathrm{~min}$, as evidenced by a mirror image of the difference spectra. Each difference spectrum was calculated from two spectra constructed from 128 interferograms taken before and after the illumination. Twenty-four $\left(\mathrm{H}_{2} \mathrm{O}\right.$ and $\left.\mathrm{D}_{2} \mathrm{O}\right)$ or forty-eight $\left(\mathrm{D}_{2}{ }^{18} \mathrm{O}\right)$ difference spectra were obtained and averaged to produce the $\mathrm{ASR}_{\mathrm{K}}$ minus ASR spectrum. ASR molecules are randomly oriented in the liposome film, which is confirmed by linear dichroism experiments, so we did not apply dichroic measurements using an IR polarizer. The obtained difference spectra were compared with those for BR with the window tilting angle of $53.5^{\circ}$ in the polarized measurement, where all vibrational bands are observed in the highly oriented BR molecule. 


\subsection{Accumulation of $13 C-A S R_{K}$}

We established the following conditions for the light adaptation. Hydrated films were illuminated with >560 nm light (O-58 cutoff filter; Toshiba) from a $1 \mathrm{~kW}$ halogen-tungsten lamp for 1 min at $4{ }^{\circ} \mathrm{C}$. HPLC analysis showed that the light-adapted ASR possesses $78 \% 13$-cis and $22 \%$ all-trans forms. The sample was cooled for $3 \mathrm{~min}$ after the light adaptation to allow for the complete decay of photoproducts.

Light-adapted ASR contains both all-trans and 13-cis forms, thus, its photoreactions strongly depend on the illumination wavelength. We established the following illumination conditions to obtain the $\mathrm{K}$ intermediate of $13 \mathrm{C}$-ASR $\left(13 \mathrm{C}-\mathrm{ASR}_{\mathrm{K}}\right)$ minus $13 \mathrm{C}$-ASR spectra at $77 \mathrm{~K}$. Illumination with $501 \mathrm{~nm}$ light for $1 \mathrm{~min}$ first converted $13 \mathrm{C}$-ASR to $13 \mathrm{C}$-ASR $\mathrm{K}$ together with the conversion of AT-ASR to the $\mathrm{K}$ intermediate of AT-ASR (AT-ASR ${ }_{K}$ ). Nevertheless, subsequent illumination at $>560 \mathrm{~nm}(\mathrm{O}-58$ cutoff filter; Toshiba) reverted only $13 \mathrm{C}-\mathrm{ASR}_{\mathrm{K}}$ to $13 \mathrm{C}$-ASR, whereas no photoreversion was found for AT-ASR ${ }_{K}$. In Section 2 for AT-ASR, we illuminated AT-ASR $\mathrm{K}_{\mathrm{K}}$ at $>590 \mathrm{~nm}$ for the photoreversion to AT-ASR. The present result indicates that illumination at $>560 \mathrm{~nm}$ does not change the

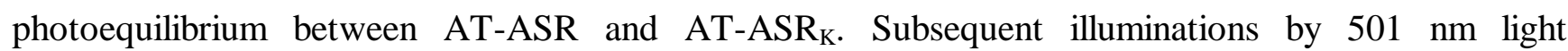
and $>560 \mathrm{~nm}$ light do not induce the spectral features of AT-ASR $\mathrm{K}_{\mathrm{K}}$ minus AT-ASR. Each difference spectrum was calculated from the two spectra constructed from 128 interferograms taken before and after the illumination.

\subsection{Accumulation of $A S R_{L}$}

Illumination with $>580 \mathrm{~nm}$ light at $170 \mathrm{~K}$ for 16 min converted the all-trans ASR to $\mathrm{ASR}_{\mathrm{L}}$. Each difference spectrum was calculated from two spectra constructed from 128 interferograms taken before and after the illumination. Three difference spectra obtained in this way were averaged to produce the $\mathrm{ASR}_{\mathrm{L}}$ minus ASR spectrum. The $\mathrm{BR}_{\mathrm{L}}$ minus BR spectra were taken from Kandori et al. [36].

\subsection{UV-Visible Spectroscopy}

The UV-visible spectra were measured by a UV-visible spectrometer (V-550, JASCO) equipped with a cryostat (OptistatDN, Oxford). The cryostat was equipped with a temperature controller (ITC-4, Oxford), and the temperature was regulated with $0.1 \mathrm{~K}$ precision. A previous HPLC study showed that the completely dark-adapted ASR in PC liposomes is in the all-trans form predominantly $(97.1 \pm 0.1 \%)$ [59]. On the other hand, illumination of ASR with $>560 \mathrm{~nm}$ light (O-58 cutoff filter, Toshiba) from a $1-\mathrm{kW}$ halogen-tungsten lamp for $1 \mathrm{~min}$ at $277 \mathrm{~K}$ yields formation of $77.9( \pm 1.7) \%$ 13-cis form [59]. Three to five independent measurements were averaged.

\subsection{HPLC Analysis}

HPLC analysis was performed as described previously [88]. A high-performance liquid chromatograph was equipped with a silica column $(6.0 \times 150 \mathrm{~mm}$; YMC-Pack SIL). The solvent was composed of $12 \%(\mathrm{v} / \mathrm{v})$ ethyl acetate and $0.12 \%(\mathrm{v} / \mathrm{v})$ ethanol in hexane, and the flow rate was $1.0 \mathrm{~mL} / \mathrm{min}$. Extraction of retinal oxime from the sample was carried out by hexane after 
denaturation by methanol and $500 \mathrm{mM}$ hydroxylamine at $4{ }^{\circ} \mathrm{C}$ [69]. The molar composition of retinal isomers was calculated from the areas of the peaks in the HPLC patterns. Assignment of the peaks was performed by comparing them with the HPLC pattern from retinal oximes of authentic all-trans and 13-cis retinals. Three independent measurements were averaged.

\section{Conclusions and Perspectives}

We studied the detail of photoreaction behavior of Anabaena sensory rhodopsin (ASR) by means of spectroscopic techniques. The results in each chapter are summarized as follows.

In Section 2, we applied low-temperature FTIR spectroscopy to the all-trans form of ASR, and compared the difference spectra at $77 \mathrm{~K}$ with those of BR. The $\mathrm{K}$ intermediate minus ASR difference spectra show that the retinal isomerizes from the all-trans to the distorted 13-cis form like BR. The N-D stretching of the Schiff base was observed at 2,163(-) and 2,125(-) $\mathrm{cm}^{-1}$, while the O-D stretchings of water molecules were observed in the $>2,500 \mathrm{~cm}^{-1}$ region. These results indicate that the protonated Schiff base forms a strong hydrogen bond with a water molecule, which is connected to Asp75 with a weak hydrogen bond. This result with ASR supports the working hypothesis by the Kandori group about the strong correlation between the proton pump activity and the existence of strongly hydrogen bonded water molecules in archaeal rhodopsins. Also we discuss the structural reason why the bridged water molecule does not form a strong hydrogen bond in ASR.

We extended the low-temperature spectroscopic study at $77 \mathrm{~K}$ to the 13-cis, 15-syn form of ASR (13C-ASR) (Section 3). HPLC analysis revealed that light-adapted ASR with light $>560 \mathrm{~nm}$ at $4{ }^{\circ} \mathrm{C}$ possesses 78\% 13C-ASR, while dark-adapted ASR has AT-ASR predominantly (97\%). Then, we established the illumination conditions to measure the difference spectra between 13C-ASR and its K state without subtracting the difference between AT-ASR and its K state. Spectral comparison between 13C-ASR and AT-ASR provided useful information on structure and structural changes upon retinal photoisomerization in ASR. In particular, previous X-ray crystallographic study of ASR reported the same protein structure for 13C-ASR and AT-ASR, whereas the present FTIR study revealed that protein structural changes upon retinal photoisomerization were significantly different between 13C-ASR and AT-ASR. The differences were seen for HOOP modes of the retinal chromophore, amide I, cysteine S-H stretch, the Schiff base N-D stretch, and water O-D stretch modes. These must trigger different global protein structural changes in each photoreaction cycle leading to the observed photochromic behavior.

ASR has been believed to function as a photoreceptor for chromatic adaptation. In this case, branching reactions, from $\mathrm{ASR}_{\mathrm{AT}}$ to $\mathrm{ASR}_{13 \mathrm{C}}$ and from $\mathrm{ASR}_{13 \mathrm{C}}$ to $\mathrm{ASR}_{\mathrm{AT}}$ (Figure 22c), are favorable for ASR, but they are in striking contrast to what is known for microbial rhodopsins. Ideally, the conversion ratios should be unity for photochromic reactions $(x=y=1$ in Figure 22c), but this is exactly the opposite of the properties of pump rhodopsins, such as BR. X-ray crystal structures reported similar chromophore structures and protein environments for $\mathrm{ASR}_{\mathrm{AT}}$ [16] and $\mathrm{BR}_{\mathrm{AT}}$ [7]. Do photochromic reactions indeed take place for $\mathrm{ASR}_{\mathrm{AT}}$ and $\mathrm{ASR}_{13 \mathrm{C}}$ ? In Section 4, we determined the branching ratios ( $\mathrm{x}$ and $\mathrm{y}$ values) for $\mathrm{ASR}_{\mathrm{AT}}$ and $\mathrm{ASR}_{13 \mathrm{C}}$ by means of low-temperature UV-visible spectroscopy. Surprisingly, the obtained $\mathrm{x}$ and $\mathrm{y}$ values were unity, indicating that the photoreactions of 
$\mathrm{ASR}_{\mathrm{AT}}$ and $\mathrm{ASR}_{13 \mathrm{C}}$ are completely photochromic. The complete photochromic reactions are highly advantageous for the chromatic sensor function of ASR.

In Section 5, we applied low-temperature FTIR spectroscopy at $170 \mathrm{~K}$ to the dark-adapted ASR that has predominantly all-trans retinal $(97 \%)$. The obtained $\mathrm{ASR}_{\mathrm{L}}$ minus ASR spectra were similar between the full-length and C-terminally truncated ASR, implying similar protein structural changes for the L state. The $\mathrm{ASR}_{\mathrm{L}}$ minus ASR spectra were essentially similar to those of $\mathrm{BR}$, but a unique spectral feature was observed in the carboxylic $\mathrm{C}=\mathrm{O}$ stretching region. The bands at $1,722(+)$ and $1,703(-) \mathrm{cm}^{-1}$ were observed at $\mathrm{pH} 5$, which was reduced at $\mathrm{pH} 7$ and disappeared at $\mathrm{pH}$ 9. The mutation study successfully assigned the bands to the $\mathrm{C}=\mathrm{O}$ stretch of Glu36. Interestingly, Glu36 is located at the cytoplasmic side, and the distance from the retinal Schiff base is about $20 \AA$ (Figure 31). We also observed $\mathrm{pH}$-dependent frequency change of a water stretching vibration, which is located near Glu36.

As shown in this review article, ASR exhibits unique photoreaction properties. They are very different from those of other archaeal-type rhodopsins, and optimized for the photochromism sensor. The X-ray crystal structure of ASR reported the similar protein architecture characteristic of archaealtype rhodopsins (Figure 4). Why is such functional optimization achieved in ASR? This is still a question that should be answered in future. Nevertheless, we suggest an important role of internal water molecules, particularly (i) at the Schiff base, and (ii) in the cytoplasmic side.

Figure 40. X-ray crystallographic structures of the Schiff base region of ASR and BR (Left). Schematic drawing of hydrogen bonds of the water molecule locating between the protonated Schiff base and its counterion (Right). This figure is reprinted with permission from TOC of Furutani et al [20]. Copyright 2005 American Chemical Society.

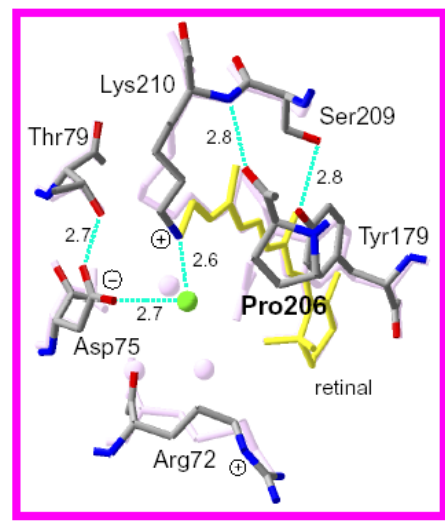

Strongly hydrogen-bonded water is present in BR (proton pump), not in ASR (no proton pump)

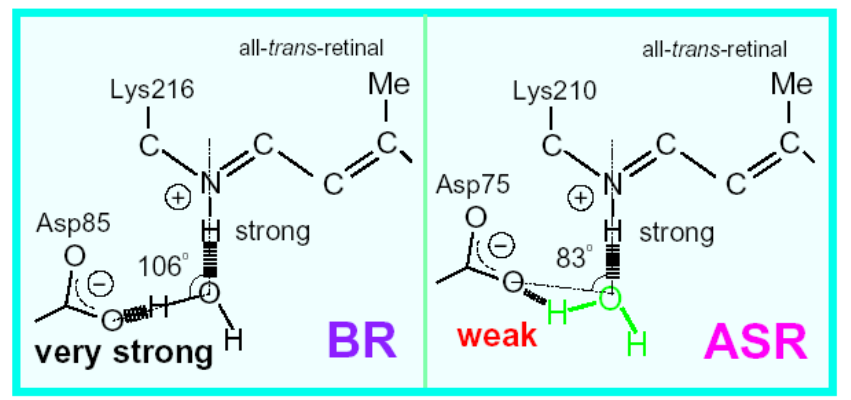

Like BR, ASR has a bridged water molecule between the Schiff base and Asp75 (Figure 4). However, Section 2 clearly showed the absence of strongly hydrogen-bonded water molecules in ASR. The reason for the lack of strongly hydrogen bonded water molecules in ASR was explained by the difference in the geometry of the hydrogen bond. Figure 40 shows that the N-Owater-OAsp75 (the Schiff base nitrogen, the water oxygen, and the oxygen of Asp75, respectively) angle in ASR is $83^{\circ}$. The corresponding N-Owater-OAsp85 angle in BR is $106^{\circ}$ (Figure 40). As the consequence, if the water oxygen fully accepts the hydrogen bond of the Schiff base, the O-H group of water points toward the oxygen of Asp85 in BR, but not toward that of Asp75 in ASR (Figure 40). Such a small difference in angle possibly determines the hydrogen bonding strength of water molecules. On the basis of our 
FTIR studies of BR mutants and other rhodopsins, we have found the strong correlation between strongly hydrogen bonded water molecules and proton pump activity. It is likely that the strong hydrogen bond of the bridged water molecule is essential for the proton-pumping function of rhodopsins, presumably because of light-energy storage through transient weakening by retinal photoisomerization. Photo-cyclic reaction is important for the efficient proton pumping in rhodopsins. Thus, weak hydrogen bond of the Schiff base water is one of the key characters in ASR.

Figure 41. X-ray crystallographic structures of BR [7] (a) and ASR [16] (b). Top and bottom panels represent views from the membrane plane and the cytoplasmic side, respectively. In the top panel, top and bottom regions correspond to the cytoplasmic and extracellular sides, respectively. The retinal chromophore is colored yellow, and green spheres represent internal water molecules.

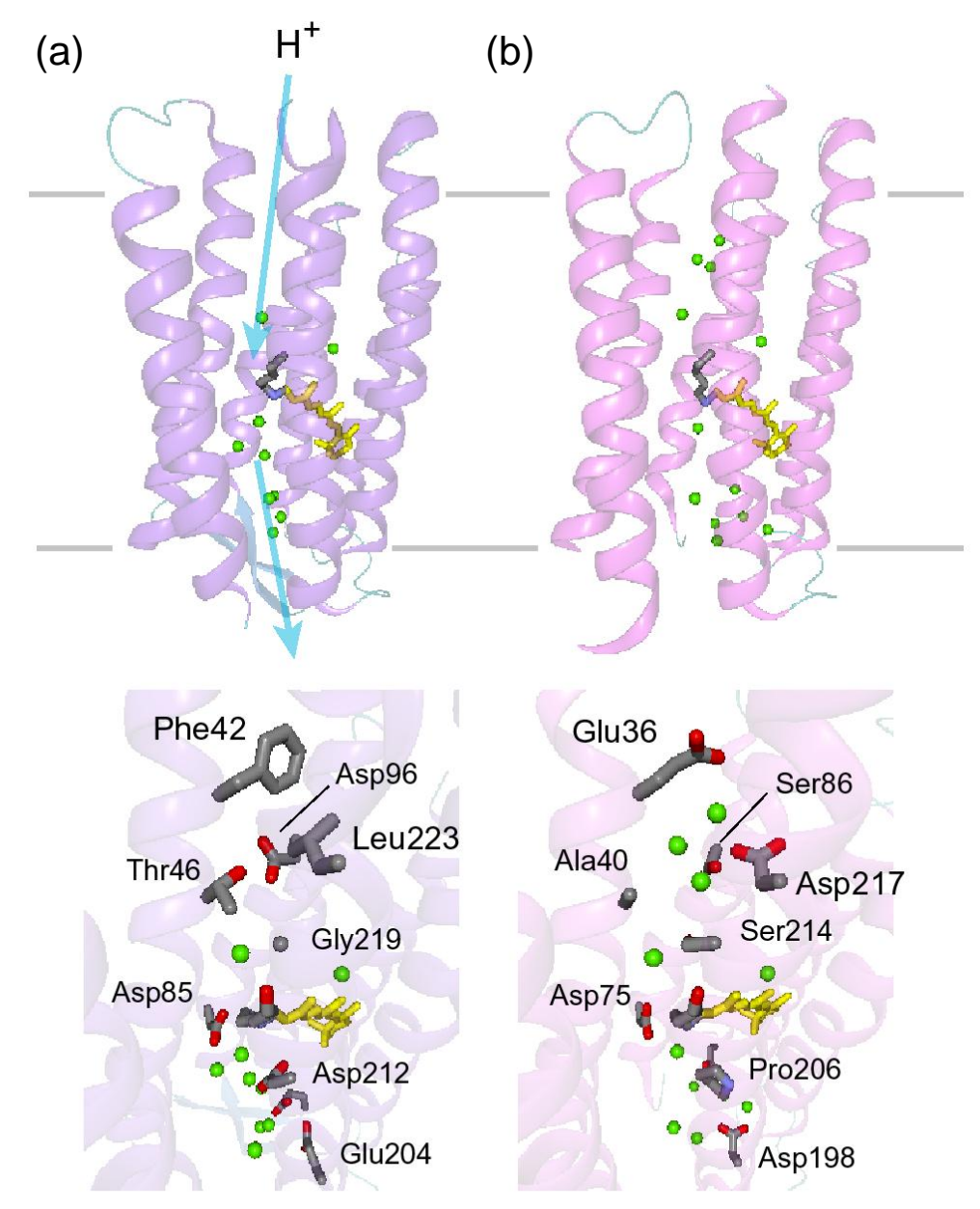

It should be however noted that many other rhodopsins do not possess strongly hydrogen-bonded water molecules, but photochromic reaction can be only seen for ASR. In this sense, polar cytoplasmic domain of ASR may play important role. Figure 41 shows that the hydrophobicity is different between the cytoplasmic and extracellular domains of BR. The cytoplasmic domain is highly hydrophobic, whereas the extracellular domain is composed of charged and polar amino acids that form a hydrogen-bonding network. Figure 41a shows the presence of 7-8 water molecules in the extracellular domain, but only 2 water molecules in the cytoplasmic domain. Such an asymmetric hydrogen-bonded 
network could be the reason of unidirectional proton transport in BR, where the proton transfer to the extracellular side occurs in $10^{-5}$ seconds, followed by reprotonation through a transiently formed proton pathway in the cytoplasmic domain on a slower timescale $\left(10^{-4}-10^{-3}\right.$ seconds). The X-ray crystallographic structure of ASR has a similar $\alpha$-helical arrangement to that of BR, but a very different hydrogen-bonded network. Figure 41b shows that in ASR both extracellular and cytoplasmic domains contain 5 water molecules, and form hydrogen-bonded networks. Such water-containing hydrogen-bonding network in the cytoplasmic region may be important in the photochromic reaction of ASR. Further study will reveal the unique reaction mechanism of the novel and interesting rhodopsin.

\section{Acknowledgements}

We thank Kwang-Hwan Jung and Yuji Furutani for their contributions to our ASR study. This work was supported in part by grants from Japanese Ministry of Education, Culture, Sports, Science, and Technology to H.K.; and Research Fellowships from the Japan Society for the Promotion of Science for Young Scientists to A. K.

\section{References and Notes}

1. Lanyi, J.K. Understanding structure and function in the light-driven proton pump bacteriorhodopsin. J. Struct. Biol. 1998, 124, 164-178.

2. Essen, L.O. Halorhodopsin: light-driven ion pumping made simple? Curr. Opsin. Struct. Biol. 2002, 12, 516-522.

3. Kamo, N.; Shimono, K.; Iwamoto, M.; Sudo, Y. Photochemistry and photoinduced proton-transfer by pharaonis phoborhodopsin. Biochemistry (Mosc) 2001, 66, 1277-1282.

4. Sasaki, J.; Spudich, J.L. Proton transport by sensory rhodopsins and its modulation by transducer-binding. Biochim. Biophys. Acta 2000, 1460, 230-239.

5. Haupts, U.; Tittor, J.; Oesterhelt, D. Closing in on bacteriorhodopsin: progress in understanding the molecule. Annu. Rev. Biophys. Biomol. Struct. 1999, 28, 367-399.

6. Lanyi, J.K. Molecular Mechanism of Ion Transport in Bacteriorhodopsin: Insights from Crystallographic, Spectroscopic, Kinetic, and Mutational Studies. J. Phys. Chem. B 2000, 104, 11441-11448.

7. Luecke, H.; Schobert, B.; Richter, H.T.; Cartailler, J.P.; Lanyi, J.K. Structure of Bacteriorhodopsin at 1.55 Å Resolution. J. Mol. Biol. 1999, 291, 899-911.

8. Bieszke, J.A.; Braun, E.L.; Bean, L.E.; Kang, S.; Natvig, D.O.; Borkovich, K.A. The nop-1 gene of Neurospora crassa encodes a seven transmembrane helix retinal-binding protein homologous to archaeal rhodopsins. Proc. Natl. Acad. Sci. USA 1999, 96, 8034-8039.

9. Sineshchekov, O.A.; Jung, K.H.; Spudich, J.L. Two rhodopsins mediate phototaxis to low- and high-intensity light in Chlamydomonas reinhardtii. Proc. Natl. Acad. Sci. USA 2002, 99, 8689-8694.

10. Nagel, G.; Ollig, D.; Fuhrmann, M.; Kateriya, S.; Musti, A.M.; Bamberg, E.; Hegemann, P. Channelrhodopsin-1: a light-gated proton channel in green algae. Science 2002, 296, 2395-2398. 
11. Okamoto, O.K.; Hastings, J.W. Novel dinoflagellate clock-related genes identified through microarray analysis. J. Phycol. 2003, 39, 519-526.

12. Jung, K.H.; Spudich, J.L. Microbial rhodopsins: Transport and sensory proteins throughout the three domains of life. In CRC Handbook of Organic Photochemistry and Photobiology, 2nd Ed.; Horspool, W.M., Lenci, F., Eds.; CRC: Boca Raton, FL, USA, 2004; pp. 124:1-124:11.

13. de la Torre, J.R.; Christianson, L.M.; Beja, O.; Suzuki, M.T.; Karl, D.M.; Heidelberg, J.; DeLong, E.F. Proteorhodopsin genes are distributed among divergent marine bacterial taxa. Proc. Natl. Acad. Sci. USA 2003, 100, 12830-12835.

14. Beja, O.; Aravind, L.; Koonin, E.V.; Suzuki, M.T.; Hadd, A.; Nguyen, L.P.; Jovanovich, S.B.; Gates, C.M.; Feldman, R.A.; Spudich, J.L.; Spudich, E.N.; DeLong, E.F. Bacterial rhodopsin: evidence for a new type of phototrophy in the sea. Science 2000, 289, 1902-1906.

15. Jung, K.H.; Trivedi, V.D.; Spudich, J.L. Demonstration of a sensory rhodopsin in eubacteria. Mol. Microbiol. 2003, 47, 1513-1522.

16. Vogeley, L.; Sineshchekov, O.A.; Trivedi, V.D.; Sasaki, J.; Spudich, J.L.; Luecke, H. Anabaena sensory rhodopsin: a photochromic color sensor at 2.0 A. Science 2004, 306, 1390-1393.

17. Vogeley, L.; Trivedi, V.D.; Sineshchekov, O.A.; Spudich, E.N.; Spudicu, J.L.; Luecke, H. Crystal structure of the Anabaena sensory rhodopsin transducer. J. Mol. Biol. 2007, 367, 741-751.

18. Grossman, A.R.; Bhaya, D.; He, Q. Tracking the light environment by cyanobacteria and the dynamic nature of light harvesting. J. Biol. Chem. 2001, 276, 11449-11452.

19. Guex, N.; Peitsch, M.C. SWISS-MODEL and the Swiss-PdbViewer: an environment for comparative protein modeling. Electrophoresis 1997, 18, 2714-23.

20. Furutani, Y.; Kawanabe, A.; Jung, K.H.; Kandori, H. FTIR spectroscopy of the all-trans form of Anabaena sensory rhodopsin at $77 \mathrm{~K}$ : hydrogen bond of a water between the schiff base and Asp 75. Biochemistry 2005, 44, 12287-12296.

21. Thompson, J.D.; Higgins, D.G.; Gibson, T.J. CLUSTAL W: improving the sensitivity of progressive multiple sequence alignment through sequence weighting, position-specific gap penalties and weight matrix choice. Nucleic Acids Res. 1994, 22, 4673-80.

22. Kandori, H. Role of internal water molecules in Bacteriorhodopsin. Biochem. Biophys. Acta 2000, 1460, 177-191.

23. Kandori, H. Hydration switch model for the proton transfer in the Schiff base region of Bacteriorhodopsin. Biochem. Biophys. Acta 2004, 1658, 72-79.

24. Iwamoto, M.; Shimono, K.; Sumi, M.; Kamo, N. Positioning proton-donating residues to the Schiff-base accelerates the M-decay of pharaonis phoborhodopsin expressed in Escherichia coli. Biophys. Chem. 1999, 79, 187-92.

25. Shibata, M.; Tanimoto, T.; Kandori, H. Water molecules in the schiff base region of bacteriorhodopsin. J. Am. Chem. Soc. 2003, 125, 13312-13313.

26. Tanimoto, T.; Furutani, Y.; Kandori, H. Structural changes of water in the Schiff base region of bacteriorhodopsin: proposal of a hydration switch model. Biochemistry 2003, 42, 2300-2306.

27. Furutani, Y.; Shibata, M.; Kandori, H. Strongly hydrogen-bonded water molecules in the Schiff base region of rhodopsins. Photochem. Photobiol. Sci. 2005, 9, 661-666. 
28. Kandori, H.; Kinoshita, N.; Shichida, Y.; Maeda, A. Protein Structural Changes in Bacteriorhodopsin upon Photoisomerization As Revealed by Polarized FTIR Spectroscopy. J. Phys. Chem. B 1998, 102, 7899-7905.

29. Aton, B.; Doukas, A.G.; Callender, R.H.; Becher, B.; Ebrey, T.G. Resonance Raman studies of the purple membrane. Biochemistry 1997, 16, 2995-2999.

30. Rothschild, K.J.; Bousche, O.; Braiman, M.S.; Hasselbacher, C.A.; Spudich, J.L. Fourier transform infrared study of the halorhodopsin chloride pump. Biochemistry 1988, 27, 2420-2424.

31. Smith, S.O.; Braiman, M.S.; Myers, A.B.; Pardoen, J.A.; Courtin, J.M.L.; Winkel, C.; Lugtenburg, J.; Mathies, R. A. Vibrational Analysis of the all-trans-Retinal Chromophore in Light-Adapted Bacteriorhodopsin. J. Am. Chem. Soc. 1987, 109, 3108-3125.

32. Braiman, M.; Mathies, R. Resonance Raman spectra of bacteriorhodopsin's primary photoproduct: evidence for a distorted 13-cis retinal chromophore. Proc. Natl. Acad. Sci. U. S. A. 1982, 79, 403-407.

33. Roepe, P.D.; Ahl, P.L.; Herzfeld, J.; Lugtenburg, J.; Rothschild, K.J. Tyrosine Protonation Changes in Bacteriorhodopsin. J. Biol. Chem. 1988, 263, 5110-5117.

34. Maeda, A.; Sasaki, J.; Pfefferle, J.M.; Shichida, Y.; Yoshizawa, T. Fourier transform infrared spectral studies on the Schiff base mode of all-trans bacteriorhodopsin and its photointermediates, K and L. Photochem. Photobiol. 1991, 54, 911-921.

35. Maeda, A.; Balashov, S.P.; Lugtenburg, J.; Verhoeven, M.A.; Herzfeld, J.; Belenky, M.; Gennis, R.B.; Tomson, F.L.; Ebrey, T.G. Interaction of Internal Water Molecules with the Schiff Base in the L Intermediate of the Bacteriorhodopsin Photocycle. Biochemistry 2002, 41, 3803-3809.

36. Kandori, H.; Shimono, K.; Sudo, Y.; Iwamoto, M.; Shichida, Y.; Kamo, N. Structural changes of pharaonis phoborhodopsin upon photoisomerization of the retinal chromophore: infrared spectral comparison with bacteriorhodopsin. Biochemistry 2001, 40, 9238-46.

37. Furutani, Y.; Bezerra, A.G.; Jr.; Waschuk, S.; Sumii, M.; Brown, L.S.; Kandori, H. FTIR Spectroscopy of the K Photointermediate of Neurospora Rhodopsin: Structural Changes of the Retinal, Protein, and Water Molecules after Photoisomerization. Biochemistry 2004, 43, 9636-9646.

38. Lin-Vien, D.; Colthup, N.B.; Fateley, W.G.; Grasselli, J.G. The Handbook of Infrared and Raman Characteristic Frequencies of Organic Molecules; Academic Press: San Diego, CA, USA, 1991.

39. Rodman-Gilson, H.S.; Honig, B.; Croteau, A.; Zarrilli, G.; Nakanishi, K. Analysis of the factors that influence the $\mathrm{C}=\mathrm{N}$ stretching frequency of polyene Schiff bases. Implications for bacteriorhodopsin and rhodopsin. Biophys. J. 1988, 53, 261-269.

40. Baasov, T.; Friedman, N.; Sheves, M. Factors Affecting the C=N Stretching in Protonated Retinal Schiff Base: A Model Study for Bacteriorhodspsin and Visual Pigments? Biochemistry 1987, 26, 3210-3217.

41. Kandori, H.; Belenky, M.; Herzfeld, J. Vibrational frequency and dipolar orientation of the protonated Schiff base in bacteriorhodopsin before and after photoisomerization. Biochemistry 2002, 41, 6026-6031.

42. Braiman, M. S.; Mogi, T.; Marti, T.; Stern, L. J.; Khorana, H. G.; Rothschild, K. J. Vibrational spectroscopy of Bacteriorhodopsin mutants: Light-driven proton transport involves protonation changes of aspartic acid residues 85, 96, and 212. Biochemistry 1988, 27, 8516-8520. 
43. Kandori, H.; Shimono, K.; Shichida, Y.; Kamo, N. Interaction of Asn105 with the Retinal Chromophore during Photoisomerization of pharaonis Phoborhodopsin. Biochemistry 2002, 41, 4554-4559.

44. Krimm, S.; Dwivedi, A.M. Infrared spectrum of the purple membrane: clue to a proton conduction mechanism? Science 1982, 216, 407-408.

45. Yamazaki, Y.; Tuzi, S.; Saito, H.; Kandori, H.; Needleman, R.; Lanyi, J.K.; Maeda, A. Hydrogen Bonds of Water and $\mathrm{C}=\mathrm{O}$ Groups Coordinate Long-Range Structural Changes in the L Photointermediate of Bacteriorhodopsin. Biochemistry 1996, 35, 4063-4068.

46. Kandori, H.; Shichida, Y. Direct Observation of the Bridged Water Stretching Vibrations Inside a Protein. J. Am. Chem. Soc. 2000, 122, 11745-11746.

47. Hayashi, S.; Tajkhorshid, E.; Kandori, H.; Schulten, K. Role of Hydrogen-Bond Network in Energy Storage of Bacteriorhodopsin's Light-Driven Proton Pump Revealed by ab Initio NormalMode Analysis. J. Am. Chem. Soc. 2004, 126, 10516-10517.

48. Kandori, H.; Yamazaki, Y.; Shichida, Y.; Raap, J.; Lugtenburg, J.; Belenky, M.; Herzfeld, J. Tight Asp-85-Thr-89 association during the pump switch of Bacteriorhodopsin. Proc. Natl. Acad. Sci. USA 2001, 98, 1571-1576.

49. Kandori, H.; Kinoshita, N.; Yamazaki, Y.; Maeda, A.; Shichida, Y.; Needleman, R.; Lanyi, J.K.; Bizounok, M.; Herzfeld, J.; Raap, J.; Lugtenburg, J. Structural Change of Threonine 89 upon Photoisomerization in Bacteriorhodopsin As Revealed by Polarized FTIR Spectroscopy. Biochemistry 1999, 38, 9676-9683.

50. Takeuchi, H.; Harada, I. Ultraviolet resonance Raman spectroscopy of X-proline bonds: A new marker band of hydrogen bonding at the imide C=O site. J. Raman Spectrosc. 1990, 21, 509-515.

51. Rothschild, K.J.; He, Y.W.; Gray, D.; Roepe, P.D.; Pelletier, S.L.; Brown, R.S.; Herzfeld, J. Fourier transform infrared evidence for proline structural changes during the bacteriorhodopsin photocycle. Proc. Natl. Acad. Sci. USA 1989, 86, 9832-9835.

52. Royant, A.; Nollert, P.; Edman, K.; Neutze, R.; Landau, E.M.; Pebay-Peyroula, E.; Navarro, J. X-ray structure of sensory rhodopsin II at 2.1-Å resolution. Proc. Natl. Acad. Sci. USA 2001, 98, 10131-10136.

53. Luecke, H.; Schobert, B.; Lanyi, J.K.; Spudich, E.N.; Spudich, J.L. Crystal Structure of Sensory Rhodopsin II at 2.4 Angstroms: Insights into Color Tuning and Transducer Interaction Science 2001, 293, 1499-1503.

54. Shibata, M.; Kandori, H. FTIR Studies of Internal Water Molecules in the Schiff Base Region of Bacteriorhodopsin. Biochemistry 2005, 44, 7406-7413.

55. Shibata, M.; Muneda, N.; Ihara, K.; Sasaki, T.; Demura, M.; Kandori, H. Internal water molecules of light-driven chloride pump proteins. Chem. Phys. Lett. 2004, 392, 330-333.

56. Furutani, Y.; Shichida, Y.; Kandori, H. Structural Changes of Water Molecules during the Photoactivation Processes in Bovine Rhodopsin. Biochemistry 2003, 42, 9619-9625.

57. Shineshchekov, O.A.; Trivedi, V.D.; Sasaki, J.; Spudich, J.L. Photochromicity of Anabaena sensory rhodopsin, an atypical microbial recepter with a cis-retinal light-adapted form. J. Biol. Chem. 2005, 280, 14663-14668.

58. Pettei, J.M.; Yudd, P.A.; Nakanishi, K.; Henselman, R.; Stoeckenius, W. Identification of retinal isomers isolated from bacteriorhodopsin. Biochemistry 1977, 16, 1955-1959. 
59. Kawanabe, A.; Furutani, Y.; Jung, K.H.; Kandori, H. FTIR study of the photoisomerization processes in the 13-cis and all-trans forms of Anabaena sensory rhodopsin at $77 \mathrm{~K}$. Biochemistry 2006, 45, 4362-4370.

60. Mathies, R.A.; Smith, S.O.; Pollard, W.T. In Biological applications of Raman spectroscopy: Resonance Raman spectra of polyenes and aromatics; Spiro, T.G., Ed.; John Wiley and Sons: New York, NY, USA, 1987; pp. 59-108.

61. Eisenberg, D.; Kauzmann, W. The Structure and Properties of Water. Oxford University: London, UK, 1969.

62. Birge, R. R. Nature of the primary photochemical events in rhodopsin and bacteriorhodopsin. Biochim. Biophys. Acta. 1990, 1016, 293-327.

63. Spudich, J.L.; Yang, C.S.; Jung, K.H.; Spudich, E.N. Retinylidene Proteins: Structures and Functions from Archaea to Humans. Annu. Rev. Cell. Dev. Biol. 2000, 16, 365-392.

64. Kandori, H. Retinal Binding Proteins: From cis-trans Isomerization in Biochemistry; Wiley-VCH: Freiburg, Germany, 2006; Chapter 4.

65. Kochendoerfer, G.G.; Lin, S.W.; Sakmar, T.P.; Mathies, R. How color visual pigments are tuned. Trends. Biochem. Sci. 1999, 24, 300-305.

66. Kandori, H.; Shichida, Y.; Yoshizawa, T. Photoisomerization in Rhodopsin. Biochemistry (Moscow) 2001, 66, 1197-1209.

67. Scherrer, P.; Mathew, M.K.; Sperling, W.; Stoecknius, W. Retinal Isomer Ratio in Dark-Adapted Purple Membrane and Bacteriorhodopsin Monomers. Biochemistry 1989, 28, 829-834.

68. Waschuk, S.K.; Bezerra, A.G., Jr.; Shi, L.; Brown, L.S. Leptosphaeria rhodopsin: Bacteriorhodopsin-like proton pump from a eukaryote. Proc. Natl. Acad. Sci. USA 2005, 102, 6879-6883.

69. Imamoto, Y.; Shichida, Y.; Hirayama, J.; Tomioka, H.; Kamo, N.; Yoshizawa, T. Chromophore Configuration of pharaonis Phoborhodopsin and Its Isomerization on Photon Absorption. Biochemistry 1992, 31, 2523-2528.

70. Tsuda, M.; Nelson, B.; Chang, C.H.; Govindjee, R.; Ebrey, T.G. Characterization of the chromophore of the third rhodopsin-like pigment of Halobacterium halobium and its photoproduct. Biophys. J. 1985, 47, 721-724.

71. Kawanabe, A.; Furutani, Y.; Jung, K.H.; Kandori, H. Photochromism of Anabaena sensory rhodopsin. J. Am. Chem. Soc. 2007,129, 8644-8649.

72. Sudo, Y.; Spudich, J. L. Three strategically placed hydrogen-bonding residues convert a proton pump into a sensory receptor. Proc. Natl. Acad. Sci. USA 2006, 103, 16129-16134.

73. Bergo, V.B.; Ntefidou, M.; Trivedi, V.D.; Amsden, J.J.; Kralj, J.M.; Rothschild, K.J.; Spudich, J.L. Conformational changes in the photocycle of Anabaena sensory rhodopsin: absence of the Schiff base counterion protonation signal. J. Biol. Chem. 2006, 281, 15208-15214.

74. Shi, L.; Yoon, S.R.; Bezerra, A.G.; Jr.; Jung, K.H.; Brown, L.S. Cytoplasmic shuttling of protons in Anabaena sensory rhodopsin: implications for signaling mechanism. J. Mol. Biol. 2006, 358, 686-700.

75. Shibata, M.; Yoshitsugu, M.; Mizuide, N.; Ihara, K.; Kandori, H. Halide binding by the D212N mutant of Bacteriorhodopsin affects hydrogen bonding of water in the active site. Biochemistry 2007, 46, 7525-7535. 
76. Sineshchekov, O. A.; Spudich, E.N.; Trivedi, V.D.; Spudich, J.L. Role of the cytoplasmic domain in Anabaena sensory rhodopsin photocycling: vectoriality of Schiff base deprotonation. Biophys. J. 2006, 91, 4519-4527.

77. Kawanabe, A.; Furutani, Y.; Jung, K.H.; Kandori, H. FTIR study of the L-intermediate of Anabaena sensory rhodopsin: Structural changes in the cytoplasmic region. Biochemistry 2008, 47, 10033-10040.

78. Gerwert, K.; Siebert, F. Evidence for light-induced 13-cis, 14-s-cis isomerization in bacteriorhodopsin obtained by FTIR difference spectroscopy using isotopically labelled retinals. EMBO J. 1986, 5, 805-811.

79. Lorenz-Fonfria, V.A.; Furutani, Y.; Kandori, H. Active internal waters in the bacteriorhodopsin photocycle. A comparative study of the $\mathrm{L}$ and $\mathrm{M}$ intermediates at room and cryogenic temperatures by infrared spectroscopy. Biochemistry 2008, 47, 4071-4081.

80. Litvin, F.F.; Balashov, S.P.; Sineshchekov, V.A. The investigation of the primary photochemical conversions of bacteriorhodopsin in purple membranes and cells of Halobacterium halobium by the low temperature spectrophotometry method. Bioorg. Khim. 1975, 1, 1767-1777.

81. Dioumaev, A.K.; Lanyi, J.K. Bacteriorhodopsin photocycle at cryogenic temperatures reveals distributed barriers of conformational substates. Proc. Natl. Acad. Sci. USA 2007, 104, 9621-9626.

82. Rammelsberg, R.; Huhn, G.; Lubben, M.; Gerwert, K. Bacteriorhodopsin's intramolecular proton-release pathway consists of a hydrogen-bonded network. Biochemistry 1998, 37, 5001-5009.

83. Garczarek, F.; Brown, L.S.; Lanyi, J.K.; Gerwert, K. Proton binding within a membrane protein by a protonated water cluster. Proc. Natl. Acad. Sci. USA 2004, 102, 3633-3638.

84. Choi, A.R.; Kim, S.Y.; Yoon, S.R.; Bae, K.; Jung, K.H. Substitution of Pro206 and Ser86 residues in the retinal binding pocket of Anabaena sensory rhodopsin is not sufficient for proton pumping function. J. Microbiol. Biotechnol. 2007, 17, 138-145.

85. Jung, K.H.; Spudich, J.L. Protonatable residues at the cytoplasmic end of transmembrane helix-2 in the signal transducer HtrI control photochemistry and function of sensory rhodopsin I. Proc. Natl Acad. Sci. USA 1996, 93, 6557-6561.

86. Jung, K.H.; Spudich, E.N.; Trivedi, V.D.; Spudich, J.L. An archaeal photosignal-transducing module mediates phototaxis in Escherichia coli. J. Bacteriol. 2001, 183, 6365-6371.

87. Shimono, K.; Furutani, Y.; Kamo, N.; Kandori, H. Vibrational modes of the schiff base in pharaonis phoborhodopsin. Biochemistry 2003, 42, 7801-7806.

88. Shimono, K.; Ikeura, Y.; Sudo, Y.; Iwamoto, M.; Kamo, N. Environment around the chromophore in pharaonis phoborhodopsin: mutation analysis of the retinal binding site. Biochim. Biophys. Acta 2001, 1515, 92-100.

(C) 2009 by the authors; licensee Molecular Diversity Preservation International, Basel, Switzerland. This article is an open-access article distributed under the terms and conditions of the Creative Commons Attribution license (http://creativecommons.org/licenses/by/3.0/). 